\title{
Information-Sharing Outage-Probability Analysis of Vehicular Networks
}

\author{
Chunxiao Jiang, Senior Member, IEEE, Haijun Zhang, Member, IEEE, Zhu Han, Fellow, IEEE, \\ Yong Ren, Senior Member, IEEE, Victor C. M. Leung, Fellow, IEEE, and Lajos Hanzo, Fellow, IEEE
}

\begin{abstract}
In vehicular networks, information dissemination/sharing among vehicles is of salient importance. Although diverse mechanisms have been proposed in the existing literature, the related information credibility issues have not been investigated. Against this background, in this paper, we propose a credible information-sharing mechanism capable of ensuring that the vehicles do share genuine road traffic information (RTI). We commence with the outage-probability analysis of information sharing in vehicular networks under both a general scenario and a specific highway scenario. Closed-form expressions are derived for both scenarios, given the specific channel settings. Based on the outage-probability expressions, we formulate the utility of RTI sharing and design an algorithm for promoting the sharing of genuine RTI. To verify our theoretical analysis and the proposed mechanism, we invoke a real-world dataset containing the locations of Beijing taxis to conduct our simulations. Explicitly, our simulation results show that the spatial distribution of the vehicles obeys a Poisson point process, and our proposed credible RTI sharing mechanism is capable of ensuring that all vehicles indeed do share genuine RTI with each other.
\end{abstract}

Index Terms-Credibility, information dissemination, information sharing, Poisson point process (PPP), reinforcement learning, vehicular networks.

V EHICULAR communications and their support networks were originally proposed for public safety applications and traffic efficiency enhancements, which necessitate reliable short-distance vehicle-to-vehicle and vehicle-to-infrastructure communications [1]. With the advent of advanced automobile technology, the globe's population has

Manuscript received September 4, 2015; revised February 3, 2016 and May 24, 2016; accepted September 26, 2016. Date of publication; date of current version. This work was supported in part by the National Natural Science Foundation China under Project 61371079 and Project 61471025 and in part by the U.S. National Science Foundation under Grant CPS-1646607, Grant ECCS1547201, Grant CCF-1456921, Grant CNS-1443917, Grant ECCS-1405121, and Grant NSFC61428101. The review of this paper was coordinated by the Editors of CVS TVT.

C. Jiang is with the Tsinghua Space Center, Tsinghua University, Beijing 100084, China (e-mail: jchx@tsinghua.edu.cn).

H. Zhang and V. C. M. Leung are with the Department of Electrical and Computer Engineering, The University of British Columbia, Vancouver, BC V6T 1Z4, Canada (e-mail: dr.haijun.zhang@ieee.org; vleung@ece.ubc.ca).

Z. Han is with the Department of Electrical and Computer Engineering and the Department of Computer Science, University of Houston, Houston, TX 77004 USA (e-mail: zhan2@uh.edu).

Y. Ren is with the Department of Electronic Engineering, Tsinghua University, Beijing 100084, China (e-mail: reny @tsinghua.edu.cn).

L. Hanzo is with the School of Electronic and Computer Science, University of Southampton, Southampton SO17 1BJ, U.K. (e-mail: 1h@ecs.soton.ac.uk).

Color versions of one or more of the figures in this paper are available online at http://ieeexplore.ieee.org.

Digital Object Identifier 10.1109/TVT.2016.2614369 become more mobile. For example, Americans ride 224 miles or more per week either as a driver or passenger, and the total time spent traveling in a vehicle per week is a staggering $18 \mathrm{~h}$ and $31 \mathrm{~min}$ [2]. Meanwhile, the vehicular users' demands for in-car communication have also been dramatically increasing, since a wealth of value-added services emerge such as safety message dissemination and in-car entertainment services.

Most of the existing works on information dissemination/sharing were focused on designing specific mechanisms, in particular scenarios of vehicular networks. However, the credibility of the shared road traffic information (RTI) has not been taken into account in those mechanisms. Although all the vehicles act in a cooperative manner, the selfish or malicious ones may share either random or manipulated information for the sake of attaining an unfair road priority. Hence, we consider this problem and propose a mechanism for ensuring that all vehicles share genuine RTI. Furthermore, we define the utility functions of vehicles in the RTI sharing mechanism for the sake of analyzing their incentives in the RTI sharing process, and provide a general analytical framework for the information-sharing outage probability (OP) of vehicular networks. The new contributions of this paper can be summarized as follows.

1) We derive the information-sharing $\mathrm{OP}$ of vehicular networks both for the general scenario modeled by Nakagami- $m$ fading and for a more specific highway scenario, where Rayleigh fading is considered.

2) In order to encourage vehicles to share genuine RTI, we design a mechanism based on the reinforcement learning model, where the concept of "reputation" is introduced for circumventing the vehicles" selfish behaviors by exploiting its similarity to human social networks.

3) The real-world dataset containing the locations of Beijing taxis is utilized for verifying the vehicles' spatial distribution characteristics. Based on the parameters inferred with the aid of training from this dataset, we verify our analytical outage performance results as well as the proposed mechanism by our real-world data-driven simulations.

The rest of the paper is organized as follows. We first summarize the related works in Section II. Then, our system model is introduced in Section III. Based on the system model, the information-sharing OP is derived both for the general Nakagami- $m$ as well as for the more specific Rayleighdistributed highway scenario in Sections IV and V, respectively. In Section VI, we present the proposed RTI sharing scheme, while Section VII provides our real-world data-driven simulation results. Finally, we conclude in Section VIII. 


\section{RELATED WORKS}

The provision of information dissemination/sharing among vehicles is of pivotal significance in vehicular networks, which has been extensively studied in the literature [3]-[21]. Specifically, Zhao etal. [3] proposed an architecture and analyzed the dissemination capacity, where the data emanating from the sources were buffered by vehicles and then it was rebroadcast at the intersections. Similarly, the concept of a "smart road" was introduced and an integrated vehicular system was conceived for the collection, management, and provision of context-aware information concerning the traffic density and driver location [4].

Later, the vehicular ad hoc network (VANET) concept was proposed for assisting the dissemination of critical vehicle tracking information [5]. Meanwhile, Cenerario et. al. designed an event-related information exchange/sharing protocol relying on a VANET in [6]. With the goal of supporting a wide range of vehicular networks, Ros et al. [7] proposed a broadcast algorithm relying on periodic beacon messages, which contained acknowledgments of the circulated broadcast messages. The urban scenario of vehicular networks was studied based on the road map information as prior knowledge in [8] and relying on peer-to-peer (P2P) cooperative caching in [9]. The heterogeneity of radio propagation was taken into account in [10], where the tradeoffs amongst parameters, such as the cost, delay, and optimized system utility, were analyzed. The performance analysis of information sharing in vehicular networks was carried out in [11]-[15]. More specifically, the distribution of concurrent transmissions was analyzed in [11], while the analysis of packet loss rate and packet transmission distance was provided in [12]. The analysis of end-to-end reliability was disseminated in [13], while the throughput and delay analysis was the subject of [14] and [15].

On the other hand, the security issues of vehicular information dissemination were investigated in [16]-[18]. Explicitly, a graph-based metric was proposed for insider attacker detection in [16], whilst a trustworthiness verification model was advocated in [17] and a cooperative neighbor position verification model was conceived in [18]. Moreover, the information sharing in vehicular networks was modeled by carefully adapting the perspective of social networks [19]-[21]. Most of the aforementioned contributions were focused on designing specific mechanisms for information dissemination/sharing in particular scenarios of vehicular networks. However, the credibility of the shared RTI has not been taken into account in those mechanisms, which hence inspired this paper.

\section{SYSTEM MODEL}

As illustrated in Fig. 1, we consider a cooperative vehicular network constituted by a group of vehicles denoted by $\mathcal{S}=\left\{v_{0}, v_{1}, v_{2}, \ldots, v_{i}, \ldots\right\}$. Since all the vehicles are independent of each other, although their locations are geographically constrained by the mesh of roads in a city, they can be viewed as being randomly distributed. By exploiting this property, we assume that the locations of the vehicles obey a Poisson point process (PPP) on the 2-D road mesh with an intensity of $\lambda$ (the number of vehicles per square kilometer). The PPP has been

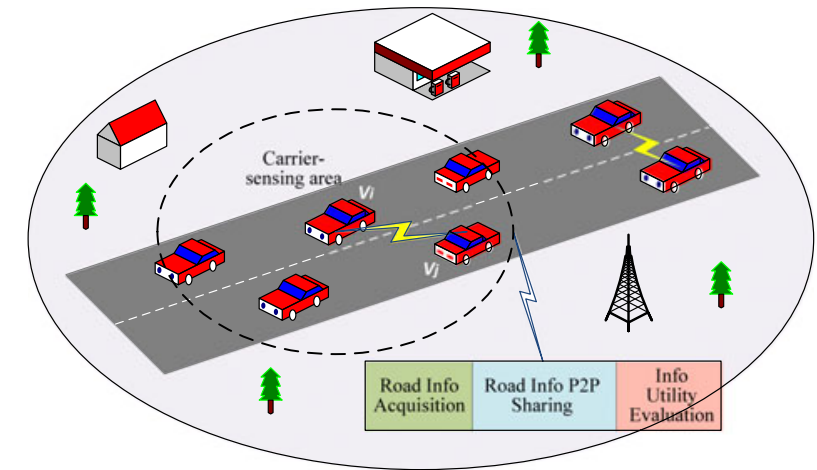

Fig. 1. System model.

widely adopted for modeling the distribution of random placements, such as the locations of macrocell and femtocell base stations [22], [23], as well as of ad hoc nodes [24]. In contrast to the existing PPP model of an infinite 2-D plane, the PPP model of a vehicular network is constrained by the road-width, which may nonetheless be as wide as say $100 \mathrm{~m}$ in metropolitan areas. Let us denote the road-width by $W$, which is assumed to be a constant. Based on the PPP model, the number of vehicles in any finite rectangle having a width of $W$ and a length of $D$ is Poisson distributed with a mean of $\lambda A_{r}$, which can be expressed as

$$
P\left(N_{r}=n\right)=\frac{e^{-\lambda W D}(\lambda W D)^{n}}{n !} .
$$

In our model, all the vehicles are assumed to be selfish, aiming for maximizing their own utility. We also assume that each vehicle has the capability of acquiring RTI and that they are willing to share it with each other in order to make better-informed decisions. The RTI can be for example the location information invoked for cooperative vehicle localization [25], or the traffic information invoked for cooperative route planning [26]. Our proposed model is general, and hence, it is independent of the specific form of the RTI. As shown in Fig. 1, at the beginning of each time slot, all the vehicles acquire the current RTI by their in-car sensors or by exploiting the driver's judgment. Then, each vehicle has to decide, whether it will truthfully share this information with others or whether to manipulate the shared RTI to render it useless, either, for example, due to privacy concerns or with the objective of gaining an unfair road priority. Therefore, although all the vehicles act in a cooperative manner, they occasionally may share random or manipulated information for the sake of improving their own utility. Then, each vehicle exchanges either its perceived genuine information or the false RTI with the nearest vehicle in a P2P mode. Following the information-sharing phase, each vehicle exploits its own information, as well as the shared information to make an informed decision as to whether to change speed, lanes, routes, or just maintain the current status. Finally, at the end of each time slot, the vehicle evaluates the performance attained as a result of its decision and then adjusts its actions in preparation for the next round. Here, we consider a practical scenario, where a vehicle is unable to ascertain the credibility of the RTI gleaned, until the 
information is actually utilized for its decision making and until the resultant performance is evaluated. Note that the time slot mentioned in this paper represents a coarse scale, on the order of seconds or minutes. Such a coarse synchronization can be readily achieved by the GPS, which has been widely deployed in vehicles. When it comes to information sharing between two vehicles, a fine-grained physical layer synchronization should be guaranteed for successful data transmission. However, such a fine-grained synchronization is not required for the entire network.

The above-mentioned P2P mode is assumed to be supported by the IEEE 802.11 p protocol (a.k.a., the Wireless Access in the Vehicular Environment (WAVE)) relying on the classic Request To Send/Clear To Send (RTS/CTS) mechanism for the sake of avoiding the hidden terminal problem [27][28]. In this case, as shown in Fig. 1, only a single pair of vehicles is sharing information in a time slot within their carrier-sensing range, such as $v_{i}$ and $v_{j}$. Based on this characteristic, the two-directional outage analysis is not considered in this paper, since only a single pair of vehicles is engaged in communication within the range. Nevertheless, the vehicles beyond $v_{i}$ and $v_{j}$ 's carrier-sensing area may also impose interference on their communications according to the practical interference model of [29]. According to the experimental results of [30], the $5.9 \mathrm{GHz}$ dedicated short-range communications frequency band may be modeled by a Nakagami- $m$ fading channel, provided that the distance between two vehicles is below $40 \mathrm{~m}$. By contrast, it is modeled by a Rayleigh-fading channel when it is above $40 \mathrm{~m}$, which is a special case of the Nakagami- $m$ fading associated with $m=1$. A line-of-sight (LOS) Rician channel may also occur under certain circumstances. Nevertheless, we would like to concentrate on the Nakagami- $m$ and Rayleigh-fading scenarios, especially when it comes to the metropolitan areas, where the presence of buildings and of the infrastructure may block the LOS as in Beijing city. Thus, the power received by the vehicle $v_{i}$ from its peer $v_{j}$ located at a distance of $d_{i, j}$ can be expressed as

$$
y_{i, j}=\left|h_{i, j}\right|^{2} d_{i, j}^{-\alpha_{i, j}}
$$

where $\alpha_{i, j}$ is the channel's path loss coefficient and $h_{i, j}$ is the channel gain. Since the distance between a pair of communicating vehicles can be $40 \mathrm{~m}$ or higher, $h_{i, j}$ should obey the Nakagami- $m$ distribution of [31]:

$$
f_{h_{i, j}}(x)=2\left(\frac{m}{\mu_{i, j}}\right)^{m} \frac{x^{2 m-1}}{\Gamma(m)} \exp \left(-m \frac{x^{2}}{\mu_{i, j}}\right)
$$

where $\Gamma(\cdot)$ is the gamma function, $\mu_{i, j}=\mathbb{E}\left(\left|h_{i, j}\right|^{2}\right)$ is the average received power, and $m$ is the Nakagami- $m$ fading parameter. In this paper, we only consider integer $m$ values for the sake of mathematical tractability. Let us introduce $g_{i, j}=\left|h_{i, j}\right|^{2}$, where $g_{i, j}$ obeys the gamma distribution of

$$
f_{g_{i, j}}(x)=\left(\frac{m}{\mu_{i, j}}\right)^{m} \frac{x^{m-1}}{\Gamma(m)} \exp \left(-m \frac{x}{\mu_{i, j}}\right) .
$$

When using the IEEE $802.11 \mathrm{p}$ protocol, all the vehicles that impose interference on the vehicle $v_{i}$ in Fig. 1 should be located farther than $40 \mathrm{~m}$ [30]. In this case, the Rayleigh-fading model should be considered for the link imposing interference by the vehicle $v_{k}$ upon $v_{i}$, i.e., $g_{i, k}$ should obey the exponential distribution of

$$
f_{g_{i, k}}(x)=\frac{1}{\mu_{i, k}} \exp \left(-\frac{x}{\mu_{i, k}}\right) .
$$

\section{Channel-Induced Outage Probability in a General SCENARIO}

In this section, we theoretically analyze the channel-induced $\mathrm{OP}$ of vehicular networks. The classic channel-induced OP of a specific vehicle $v_{i}$ is defined as the probability of $v_{i}$ 's signal-tointerference-plus-noise ratio (SINR) dipping below a threshold of $\Upsilon$, i.e.,

$$
p_{v_{i}}=\mathbb{P}\left[\gamma_{v_{i}} \leq \Upsilon\right]
$$

which, in fact, is also the cumulative distribution function (c.d.f) 234 of this vehicle's SINR. Since the channel-induced OP is a 235 physical-layer metric, the fact of whether a vehicle shares gen- 236 uine or false information is irrelevant in this section. By contrast, 237 in Section V, we will use the channel-induced OP for modeling 238 the vehicles' future utility trend, depending on whether they are 239 sharing genuine or false RTI.

As illustrated in the system model, we consider a P2P 241 scenario, where every pair of closest vehicles exchange their 242 respective RTI within each time slot. For a specific vehicle $v_{0}, \quad 243$ its closest counterpart $v_{1}$ should be the intended information- 244 sharing peer. Let us denote the distance and channel gain of $v_{0} \quad 245$ with respect to the transmitter of the vehicle $v_{1}$ by $d_{1}$ and $g_{1}, \quad 246$ respectively. Then, the SINR of the vehicle $v_{0}$ can be written as 247

$$
\gamma_{0}=\frac{g_{1} d_{1}^{-\alpha_{1}}}{\Lambda}
$$

where $\alpha_{1}$ is the path loss coefficient, and $\Lambda$ is the interference imposed by the other vehicles on the vehicle $v_{0}$ plus the noise power. Let us assume that $v_{1}$ is the vehicle closest to $v_{0}$. Then, according to the experimental results of [30], the channel gain $g_{1}$ should obey the gamma distribution as in (4) with a mean of $\mathbb{E}\left[g_{1}\right]=\mu_{1}$ and Nakagami- $m$ fading parameter of $m_{1}$. During the information sharing between the pair of vehicles $v_{0}$ and $v_{1}$, the signals of all other vehicles, represented by $v_{i}\left(\forall v_{i} \in\right.$ $\left.\mathcal{S} \backslash\left\{v_{0}, v_{1}\right\}\right)$, should be considered as interference. Let us denote the distance and channel gain between $v_{i}$ and $v_{0}$ by $d_{i}$ and $g_{i}$, respectively. In this case, the interference plus noise power $\Lambda$ can be calculated by

$$
\Lambda=\sum_{v_{i} \in \mathcal{S} \backslash\left\{v_{0}, v_{1}\right\}} g_{i} d_{i}^{-\alpha_{2}}+\sigma^{2}
$$

where $\alpha_{2}$ is the path loss coefficient and $\sigma^{2}$ is the variance of the zero-mean circularly symmetric complex-valued Gaussian noise. Assuming that the other vehicles-except for the closest one-are relatively far from $v_{0}$, Rayleigh fading prevails between $v_{i}$ and $v_{0}$, i.e., the interfering channel's gain $g_{i}$ obeys the exponential distribution as in (5). Since all vehicles are independent of each other, the channel gains $\left\{g_{i, v_{i} \in \mathcal{S} \backslash\left\{v_{0}, v_{1}\right\}}\right\}$ are independent identically distributed (i.i.d.), where $\mathbb{E}\left[g_{i, v_{i} \in \mathcal{S} \backslash\left\{v_{0}, v_{1}\right\}}\right]=\mu_{2}$. Thus, the SINR of 39 240 41 42 4 46 247 
vehicle $v_{0}$ becomes

$$
\gamma_{0}=\frac{g_{1} d_{1}^{-\alpha_{1}}}{\sum_{v_{i} \in \mathcal{S} \backslash\left\{v_{0}, v_{1}\right\}} g_{i} d_{i}^{-\alpha_{2}}+\sigma^{2}}
$$

1 where we have

$$
\mathcal{G}_{\alpha}(x)=\int_{x}^{+\infty} \frac{1}{1+u^{\alpha / 2}} \mathrm{~d} u .
$$

Proof: See the proof in Appendix A.

\section{INFORMATION-SHARING OUTAGE PERFORMANCE IN HIGHWAY SCENARIO}

In Theorem 1, (11) provides the information-sharing OP of vehicular networks in a general form, which can be used in any arbitrary scenario, including both dense and sparse vehicular network scenarios. However, when considering specific application scenarios, further approximations can be adopted in the derivation of Theorem 1 . In this section, we will consider a highway-specific scenario, where the distance amongst vehicles may be substantially higher than in the downtown area, say over $30 \mathrm{~m}$ on average. According to the experimental results of [30], the channel between a pair of vehicles in this highway scenario is Rayleigh fading, which implies that the channel between vehicle $v_{1}$ and $v_{0}$ is Rayleigh fading. Hence, $g_{1}$ in (7) obeys follow the exponential distribution with the same mean as $g_{i}$. In essence, this specific Rayleigh-fading highway scenario constitutes a special case of Nakagami- $m$ fading associated with $m=1$. The following corollary formulates the channel-induced OP in this highway scenario.

Corollary 1: In a highway vehicular network relying on the 802.11p protocol and RTS/CTS, a vehicle's information-sharing $\mathrm{OP}$ can be expressed as

$$
\begin{aligned}
& p_{0}^{\mathrm{hwy}_{1}}=1-2 \lambda W \int_{\tau=0}^{+\infty} \exp \left(-\frac{\sigma^{2} \Upsilon}{\mu} \tau^{\alpha_{1}}\right) \\
& \exp \left[-2 \lambda W \Upsilon^{\frac{1}{\alpha_{2}}} \tau^{\frac{\alpha_{1}}{\alpha_{2}}} \mathcal{G}_{\alpha_{2}}\left(\left(\frac{\tau^{\alpha_{2}-\alpha_{1}}}{\Upsilon}\right)^{\frac{1}{\alpha_{2}}}\right)\right] \cdot e^{-2 \lambda W \tau} \mathrm{d} \tau
\end{aligned}
$$

Proof: See the proof in Appendix B.

According to the experimental results of [30], in the highway scenario the path loss measurements showed a dual-slope model, having a break-point at the distance of $100 \mathrm{~m}$. When the distance between two vehicles is below $100 \mathrm{~m}$, the path loss coefficient is $\alpha$, while beyond $100 \mathrm{~m}$ it is $\beta$. Since $100 \mathrm{~m}$ is already at the limit of the 802.11 p-based P2P information sharing, we can focus our attention on considering the scenario, where all vehicles' path loss models are identical, i.e., $\alpha_{1}=\alpha_{2}=\alpha$. Specifically, the experimental results of [30] showed that the path loss coefficient is $\alpha=2$ under $100 \mathrm{~m}$. The channel-induced OP of this specific scenario is formulated in the following corollary.

Corollary 2: In a highway vehicular network using the 802.11p protocol and RTS/CTS, where the path loss coefficients amongst the vehicles are identical, a vehicle's information-sharing OP can be expressed as

$$
\begin{aligned}
p_{0}^{\mathrm{hwy}}= & 1-2 \lambda W \int_{\tau=0}^{+\infty} \exp \\
& \times\left[-\frac{\sigma^{2} \Upsilon}{\mu} \tau^{\alpha}-2 \lambda W\left(1+\Phi_{\alpha}(\Upsilon)\right) \tau\right] \mathrm{d} \tau .
\end{aligned}
$$

Specifically, when the channel's path loss coefficient is $\alpha=2, \quad 318$ the closed-form expression of the channel-induced OP can be 319 formulated as

$$
\begin{aligned}
p_{0} \text { hwy }_{2}= & 1-2 \lambda W \sqrt{\frac{\pi}{\chi_{1}(\Upsilon)}} \exp \left(\frac{\chi_{2}^{2}(\Upsilon)}{4 \chi_{1}(\Upsilon)}\right) \\
& \times Q\left(\frac{\chi_{2}(\Upsilon)}{\sqrt{2 \chi_{1}(\Upsilon)}}\right)
\end{aligned}
$$

where $\chi_{1}(\Upsilon)$ and $\chi_{2}(\Upsilon)$ are

$$
\begin{aligned}
& \chi_{1}(\Upsilon)=\frac{\sigma^{2}}{\mu} \Upsilon \\
& \chi_{2}(\Upsilon)=2 \lambda W(1+\sqrt{\Upsilon} \arctan \sqrt{\Upsilon}) .
\end{aligned}
$$

Proof: See the proof in Appendix C.

It can be seen that (16) gives a simple closed-form expression 323 for a single vehicle's information-sharing OP, which simply re- 324 lies on the calculation of the $Q$-function. If we now consider the 325 specific scenario, where the channel noise is negligible com- 326 pared to the interference arriving from the other vehicles $v_{i}, \quad 327$ i.e., for $\sigma^{2} / \Lambda \rightarrow 0$, the information-sharing OP can be further 328 simplified using the following corollary.

Corollary 3: In a highway vehicular network associated with the 802.11p protocol and RTS/CTS, where the path loss coefficients of all vehicles are identical and the channel noise is negligible compared to the interference, a vehicle's informationsharing OP during a specific time slot can be expressed as

$$
p_{0}^{\mathrm{hwy}_{3}}=\frac{\Phi_{\alpha}(\Upsilon)}{1+\Phi_{\alpha}(\Upsilon)} .
$$

Specifically, when the channel's path loss coefficient is $\alpha=2, \quad 335$ we have

$$
p_{0}^{\mathrm{hwy}_{3}}=\frac{\sqrt{\Upsilon} \arctan \sqrt{\Upsilon}}{1+\sqrt{\Upsilon} \arctan \sqrt{\Upsilon}} .
$$


Proof: Equations (19) and (20) can be readily obtained by setting $\sigma^{2}=0$ in (15) and (16), respectively.

By now, we have completed the theoretical informationsharing OP analysis, which is an important metric that reflects whether information sharing can be reliably accomplished. Note that successful information sharing in the vehicular network relies both on successful transmission in the presence of no channel-induced outage and no genuineinformation-sharing outage. Based on the channel-induced OP analysis of this section, the next section will propose a RTI sharing mechanism that ensures for the vehicles to share genuine information.

\section{RoAD TRAFFic ENGINEERING SHARING MECHANISM}

In the previous section, we have studied the informationsharing OP of the vehicular network considered. Following the above performance analysis, this section will consider the vehicles' information-sharing strategies, utilities, and interactions during the RTI sharing process. Note that the sharing of RTI cannot succeed if a channel-induced outage happens between the vehicles. Let us consider a cooperative vehicular network supporting $N$ selfish vehicles indexed as $\left\{v_{1}, v_{2}, \ldots, v_{N}\right\}$, each aiming for maximizing its own utility. As mentioned in the introduction, although all vehicles share the RTI in a cooperative manner, their specific degree of altruism/selfishness determines whether they may share false or genuine RTI for the sake of improving their own utility by exploiting unfair priority on the road for example. Considering this issue, each vehicle $v_{i}$ is assumed to have a binary action space defined as follows:

$$
a_{i}= \begin{cases}\mathbf{S}_{G}: & \text { sharing genuine RTI } \\ \mathbf{S}_{F}: & \text { sharing false RTI. }\end{cases}
$$

As a counterpart, a mixed strategy can also be defined for vehicle $v_{i}$ in which $q_{i}$ represents the probability of vehicle $v_{i}$ sharing genuine RTI, complemented by a $\left(1-q_{i}\right)$ probability of false RTI. As mentioned in the system model, each vehicle evaluates the RTI gleaned from its peer vehicle at the end of each time slot. Additionally, we also consider a binary information reward space, where the genuine RTI earns a reward of $R$, while the issuance of false RTI results in a zero reward. In such a case, we can summarize vehicle $v_{i}$ 's utility functions as follows:

$$
\left\{\begin{array}{l}
U_{\mathrm{ij}}\left(\mathbf{S}_{G}, \mathbf{S}_{G}\right)=\left(1-p_{\mathrm{ij}}\right) R-c_{i} \\
U_{\mathrm{ij}}\left(\mathbf{S}_{G}, \mathbf{S}_{F}\right)=-c_{i} \\
U_{\mathrm{ij}}\left(\mathbf{S}_{F}, \mathbf{S}_{G}\right)=\left(1-p_{\mathrm{ij}}\right) R \\
U_{\mathrm{ij}}\left(\mathbf{S}_{F}, \mathbf{S}_{F}\right)=0
\end{array}\right.
$$

where $U_{\mathrm{ij}}(a, b)$ represents vehicle $v_{i}$ 's utility, when its strategy is $a$ and its peer $v_{j}$ 's strategy is $b$ with $p_{\mathrm{ij}}$ denoting the channelinduced OP between $v_{j}$ and $v_{i}$, and $c_{i}>0$ represents the additional cost of sharing genuine information. Then, $\left(1-p_{\mathrm{ij}}\right) R$ quantifies the expected reward. Additionally, it is assumed that the link's OP $p_{\mathrm{ij}}$ should be no higher than $1-\frac{c_{i}}{R}$; otherwise, no vehicle would share genuine RTI under any circumstances.

The credit mechanism of the vehicular networks considered may be designed by observing human social networks. The concept of "reputation" is rather important for everyone in the real world, where a person's credit/reputation is generated and updated according to his/her accumulated behaviors in human social networks. Explicitly, when interacting with a reputable person, we are inclined to maintain future contacts with him/her. On the other hand, if we learned a lesson from interacting with someone having a bad reputation, a long-lasting cooperation may be unlikely. Similarly, in our cooperative vehicular network, each vehicle can evaluate the others' credit through learning from its interactions with other vehicles. In this case, a vehicle can determine whether to share its RTI with a specific vehicle according to that vehicle's credit/reputation. When a vehicle's credit is below a certain threshold, other vehicles would not share any RTI with it. It is expected that through rounds of interactions, each vehicle's credit can be gradually learned by the observations and evaluations of its shared RTI. According to this credit information, the vehicles associated with a low credit would obtain less and less shared RTI, and eventually they will have to change their RTI sharing strategy to improve their reputation. We assume that there is a central server and each vehicle can report its experience in sharing RTI with all others. As a result, the database records the vehicles' credit. The credit established by each vehicle is considered to be private information, which may not be appropriate for the server to release to the public. This is similar to our human social network, where the credit earned by each individual is not directly visible to others. Nevertheless, through rounds of interactions, one vehicle's credit can be gradually learned by others. Note that the central server is only used by the vehicles to inform the others about their RTI sharing experience and to store the credit value of each vehicle. Since the experience can be quantized to a low number of discrete levels, the amount of data related to each vehicle is relatively small. Therefore, the server does not have to maintain a large-scale database. A potential solution is that each vehicle stores its own experience and the credit values of other vehicles locally.

Similar to the human social networks, each vehicle of our vehicular network can have a credit value generated by its past behavior, and also determines its future behavior when sharing RTI with others. Let us define vehicle $v_{i}$ ' reputation value as $r_{i}$ in conjunction with $0 \leq r_{i} \leq 1$. Note that in human social networks, a person's behavior is typically consistent with his/her reputation, regardless of the specific credit of the other persons he/she is interacting with. Similarly, vehicle $v_{i}$ 's RTI sharing strategy $q_{i}$ should also be consistent with its reputation $r_{i}$, and thus these two parameters can be deemed to be identical, i.e., we have $r_{i}=q_{i}$. When $v_{i}$ has the knowledge of vehicle $v_{j}$ 's credit/reputation through rounds of RTI sharing interactions, $v_{i}$ can determine whether to cooperate with $v_{j}$ in the future. Let us define $v_{i}$ 's interaction probability and action with respect to other vehicles as

$$
\begin{aligned}
& \boldsymbol{\kappa}_{i}=\left[\kappa_{i 1}, \kappa_{i 2}, \ldots, \kappa_{\mathrm{iN}}\right] \\
& \boldsymbol{\eta}_{i}=\left[\eta_{i 1}, \eta_{i 2}, \ldots, \eta_{\mathrm{iN}}\right]
\end{aligned}
$$

where $0 \leq \kappa_{\mathrm{ij}} \leq 1$ represents $v_{i}$ 's probability of sharing RTI 434 with $v_{j}$, regardless whether this is genuine or false information, 435 
and $\eta_{\mathrm{ij}}=0$ or 1 represents whether or not to cooperate with $v_{j}$ in a specific time slot. In such a scenario, at the beginning of each time slot, each vehicle first has to determine its next action $\eta_{\mathrm{ij}}$, i.e., whether to cooperate with the nearest vehicle $v_{j}$, according to $v_{i}$ 's interaction probability $\kappa_{\mathrm{ij}}$. Then, if it has decided to share RTI with $v_{j}$, it has to further determine the RTI sharing action $a_{i}$, i.e., as to whether to share genuine or false RTI with a specific peer vehicle, according to both $v_{i}$ 's information-sharing strategy $q_{i}$ as well as to its reputation $r_{i}$.

Meanwhile, after rounds of RTI sharing interactions, vehicle $v_{i}$ should update its interaction probability $\boldsymbol{\kappa}_{i}$ according to its experience with the others or by querying the database. It is expected that through a series of alternating decision making and learning processes, the vehicles having a bad reputation would obtain decreasingly less shared RTI from the others, and thus they would have to ameliorate their credit/reputation by actively sharing genuine RTI hereafter.

During the multiround RTI sharing process, none of the vehicles has access to the other vehicles' information-sharing strategies, actions, and utilities. Moreover, due to the rapidly evolving topology of vehicular networks, each vehicle may share its RTI with different vehicles during different time slots. Hence, from an individual vehicle's perspective, the network including all other vehicles can be regarded as an external environment, within which the vehicle makes decisions and shares RTI with the goal of maximizing its own utility. Generally, each vehicle learns from its interactions with this dynamic environment and adapts to the environment by adjusting its strategies for the sake of gleaning an increased utility. Reinforcement learning is a powerful tool capable of solving such an adaptive environment-learning and decision-making problem [32]. Its actions are reminiscent of how an intelligent agent infers the unknown statistical features of its environment as well as its actions in the environment so as to maximize a certain notion of the cumulative reward, where the environment itself is gradually changed by the agent's actions. Reinforcement learning has been widely adopted in communications and networks [33], [34], control [35], finance, and economics [36], as well as in social science [37], [38].

In our model, one of the main technical problems is how each vehicle constructs its interaction probability vector $\boldsymbol{\kappa}_{i}$ after rounds of RTI sharing interactions with the others. Based on the reinforcement learning model, each vehicle should first construct its perception through learning the others' inclination in RTI sharing. The perception is a quantitative representation of the accumulated utilities, which records all the historical interactions of the past as well as the new interaction results. In other words, it relies on the exploitation of past knowledge and on the exploration of a new environment [32]. Let us define vehicle $v_{i}$ 's perception of the others' behaviors as $\mathbf{z}_{i}$, where

$$
\mathbf{z}_{i}=\left[z_{i 1}, z_{i 2}, \ldots, z_{\mathrm{iN}}\right]
$$

with $z_{\mathrm{ij}}$ being vehicle $v_{i}$ 's perception with respect to $v_{j}$. At the end of each time slot, $v_{i}$ first evaluates the utility of information received from $v_{j}$ and then utilizes this utility value for adjusting its perception associated with $v_{j}$, while keeping the perception of others unchanged, which can be expressed as

$$
z_{\mathrm{ij}}^{t+1}= \begin{cases}\left(1-\epsilon_{i}^{t}\right) z_{\mathrm{ij}}^{t}+\epsilon_{i}^{t} U_{\mathrm{ij}}^{t}, & \text { if } \eta_{\mathrm{ij}}^{t}=1 \\ z_{\mathrm{ij}}^{t}, & \text { if } \eta_{\mathrm{ij}}^{t}=0\end{cases}
$$

where the superscript $t$ represents the time slot, $U_{\mathrm{ij}}^{t}$ is $v_{i}$ 's utility 491 gleaned through exchanging information with $v_{j}$ during time 492 slot $t$, and $\epsilon_{i}^{t}$ is a sequence of averaging factors controlling the 493 rate of decay in conjunction with $\sum_{t} \epsilon_{i}^{t}=\infty$ and $\sum_{t}\left(\epsilon_{i}^{t}\right)^{2}<\infty . \quad 494$ The constraint of $\sum_{t} \epsilon^{t}=\infty$ is imposed for ensuring $\epsilon^{t}>0,495$ i.e., the new learned utility $U_{\mathrm{ij}}^{t}$ should always be incorporated. 496 By contrast, the constraint of $\sum_{t}\left(\epsilon^{t}\right)^{2}<\infty$ is used for ensuring 497 $\epsilon^{t}<1$, i.e., the history of the learned experience $z_{\mathrm{ij}}^{t}$ should 498 always be utilized.

After updating the perception $\mathbf{z}_{i}$, vehicle $v_{i}$ can utilize it for generating its interaction probability with respect to vehicle $v_{j}$. Apparently, the more utility $v_{i}$ can obtain through sharing RTI with vehicle $v_{j}$, the higher the interaction probability $\kappa_{\mathrm{ij}}$ should be, which represents a proportional relationship between $\kappa_{\mathrm{ij}}$ and $z_{\mathrm{ij}}$. Here, we adopt a normalized performance evaluation method based on the Boltzmann exploration rule formulated as follows [32]:

$$
\kappa_{\mathrm{ij}}^{t}=\frac{e^{\xi_{i}^{t} z_{\mathrm{ij}}^{t}}}{\max \left\{e^{\xi_{i}^{t} z_{\mathrm{ij}}^{t}}, \forall j\right\}}
$$

where the positive coefficient $\xi_{j}^{t}$ controls the exploration level 508 with $\xi_{j}^{t} \rightarrow 0$ leading to a 0.5 interaction probability, while for 509 $\xi_{j}^{t} \rightarrow \infty$ the action would concentrate only on one of the pure 510 unconditional cooperation or no cooperation strategy, whichever 511 results in a higher perception. The physical meaning of (27) is 512 that vehicle $v_{i}$ always shares RTI with that specific vehicle, 513 which can give $v_{i}$ the highest utility. Then, $v_{i}$ considers this 514 highest utility as a reference, when it determines its interaction 515 probability with others.

To summarize, the reinforcement learning-based credible RTI sharing scheme can be interpreted as a process, in which each vehicle learns about its utilities as well as perceptions, and then updates its estimation regarding the other vehicles' reputation as well as adjusts its interaction behavior accordingly using its accumulated perception. The evolution from $z_{\mathrm{ij}}^{t}$ to $z_{\mathrm{ij}}^{t+1}$ can be illustrated by a chain of iterative elementary steps: the initial perception gives rise to a random interaction probability that determines the interaction; by following the interaction and the information-sharing action, the resultant utility is evaluated and then the perception can be updated in the next round, and so on. The iterations can be simply expressed by the following illustrative chain:

$$
\begin{gathered}
z_{\mathrm{ij}}^{t} \rightarrow \kappa_{\mathrm{ij}}^{t} \rightarrow \eta_{\mathrm{ij}}^{t} \rightarrow U_{\mathrm{ij}}^{t} \rightarrow z_{\mathrm{ij}}^{t+1} \\
\downarrow \\
\quad r_{i}^{t} \rightarrow q_{i}^{t} \rightarrow a_{i}^{t}
\end{gathered}
$$

where the arrow between $\kappa_{\mathrm{ij}}^{t}$ and $r_{i}^{t}$ means that when a vehicle 530 discovers that the number of other vehicles sharing RTI with it 531 is less than a certain threshold, the vehicle would consider to 532 increase its credit value in order to enhance its reputation by 533 


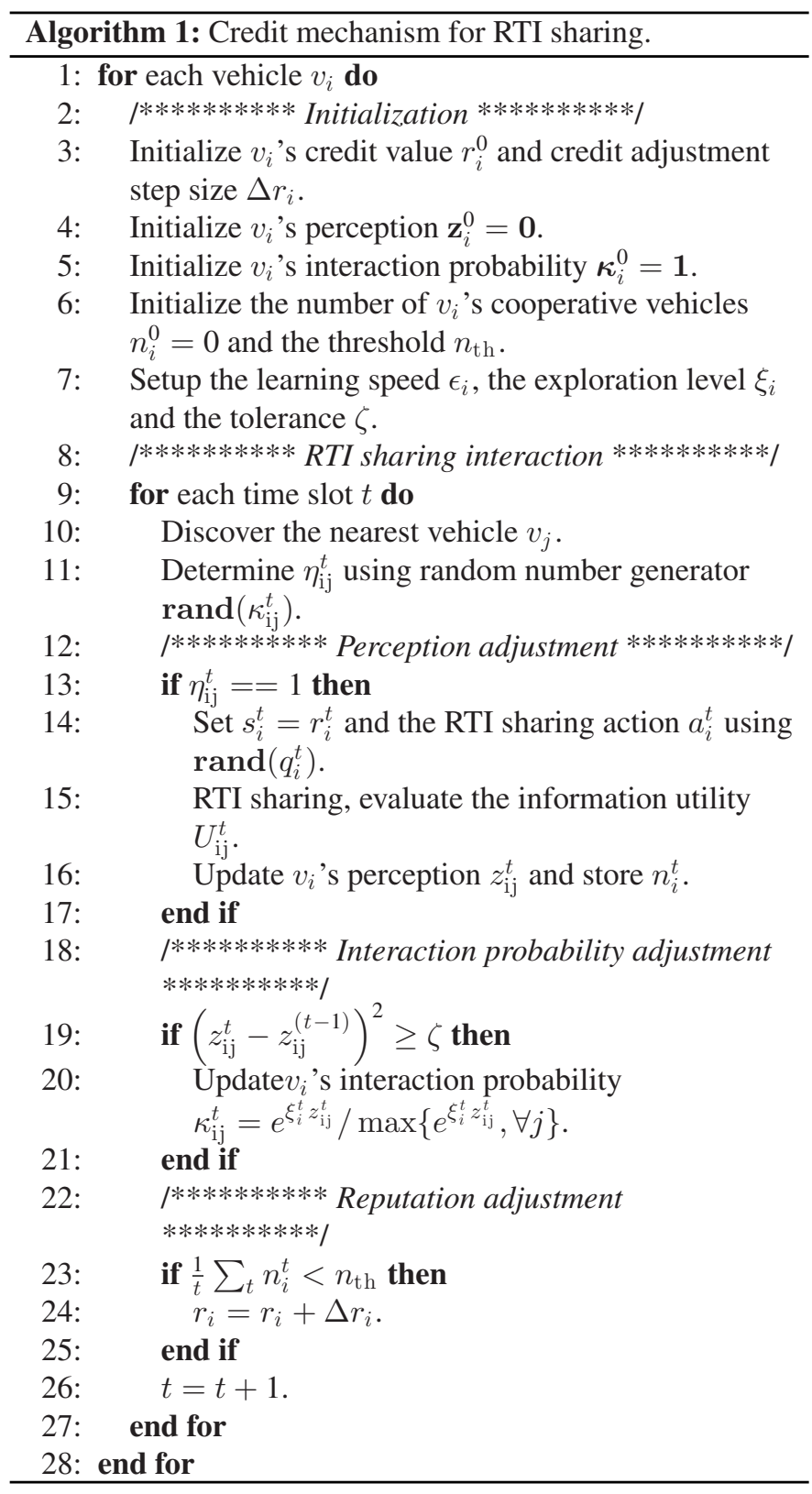

sharing more genuine RTI with the others. The credit mechanism is summarized in Algorithm 1. In the initialization phase, each vehicle may have different prior credit vales and credit adjustment preference. Meanwhile, the learning speed $\epsilon$ determines the weight of new information, the exploration level $\xi$ determines the probability of adopting uncharted strategies, while the tolerance determines the learning performance. In the RTI sharing phase, each vehicle first connects with the nearest vehicle and generates the interaction strategy, i.e., whether to interact with the vehicle. If the interaction indicator is positive, the vehicle then shares the genuine RTI with a probability generated by its reputation. Following the information-sharing interaction, the vehicle evaluates its perception and updates the interaction probability in the next round. If the vehicle finds that the number of other vehicles who would like to exchange information with it is below some threshold, the vehicle would

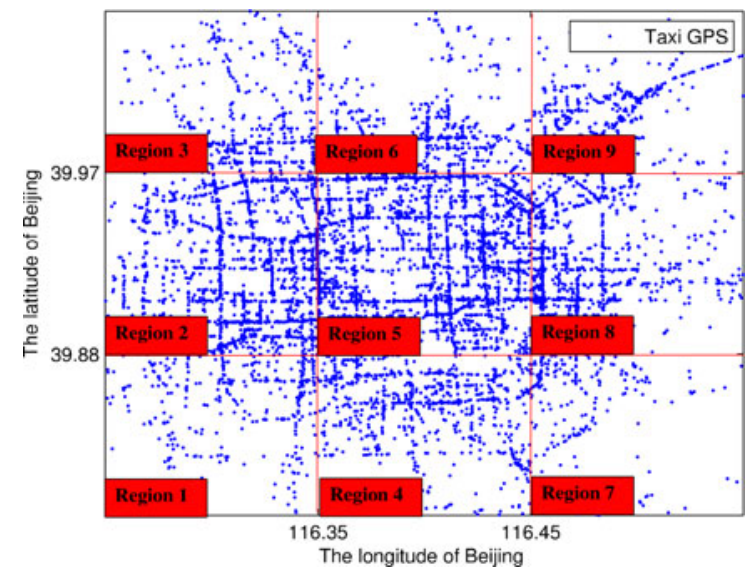

Fig. 2. Locations of Beijing taxis.

TABLE I

VeHICLE INTENSITIES OF DIFFERENT REGIONS AT BEIJING

\begin{tabular}{lccccc}
\hline \hline Region & 0 & 1 & 2 & 3 & 4 \\
\hline Intensity $\left(/ \mathrm{km}^{2}\right)$ & 59.6 & 23.3 & 72.7 & 40.7 & 48.1 \\
Average distance $(\mathrm{m})$ & 89.03 & 227.79 & 73.01 & 130.41 & 110.42 \\
$\mathrm{~K}-\mathrm{S}$ test $(P$-value $)$ & 0.0731 & 0.1179 & 0.1061 & 0.0705 & 0.0619 \\
Region & 5 & 6 & 7 & 8 & 9 \\
Intensity $\left(/ \mathrm{km}^{2}\right)$ & 76.8 & 46.3 & 21.2 & 74.4 & 59.6 \\
Average distance $(\mathrm{m})$ & 69.12 & 114.57 & 250.00 & 71.35 & 89.05 \\
K-S test $(P$-value $)$ & 0.1169 & 0.0774 & 0.0831 & 0.0584 & 0.0937 \\
\hline \hline
\end{tabular}

TABLE II

Numerical Parameters For Performance Evaluation

\begin{tabular}{lc}
\hline \hline Parameter & Value \\
\hline Max Tx Power & $20 \mathrm{dBm}$ \\
Antennas & $1 \mathrm{Tx}, 1 \mathrm{Rx}$ \\
Antennas gains & $5 \mathrm{dBm}$ \\
Nakagami- $m$ fading parameter & $m=2$ \\
Path loss exponent & $\alpha=2,4$ \\
Noise power & $\sigma^{2}=0.1 \mathrm{dBm}$ \\
Maximum OP & $\Upsilon=0.1$ \\
\hline \hline
\end{tabular}

adjust its reputation according to the preferred adjustment step 550 size. In the next section, we will conduct simulations to quantify 551 the performance of the proposed algorithm.

\section{Simulation Results Based on Real Traffic Data 553}

In this section, we conduct simulations to verify our 554 theoretical analysis and characterize the proposed schemes. The 555 simulations are based on a real-world dataset consisting of the 556 spatial distribution of Beijing taxis. In the following, we will first 557 estimate the intensity of the taxis in Beijing using the dataset. 558 Then, based on the estimated intensity, we will characterize the 559 outage performance of RTI sharing as well as verify the merits 560 of the proposed RTI sharing scheme. 561

The real-world dataset contains the GPS positions of 10258562 taxis in Beijing (longitude from 116.25 to 116.55 and latitude 563 from 39.8 to 40.05) during the period of February 2-8, 2008564 [39]. As shown in Fig. 2, the positions of these vehicles at a 565 


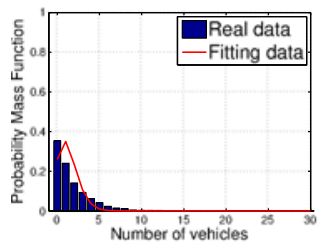

Whole Beijing.

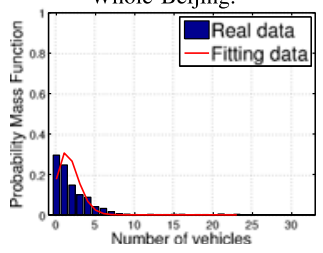

Region 5 .

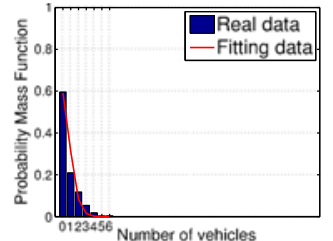

Region 1 .

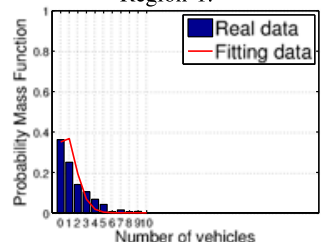

Region 6.

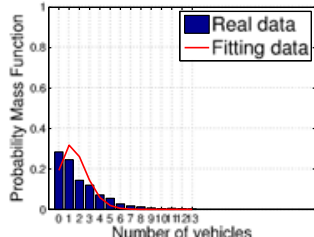

Region 2.

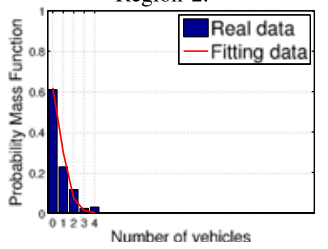

Region 7.

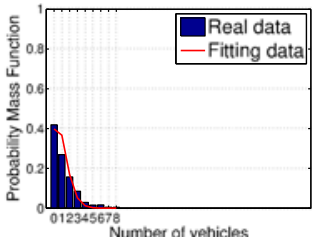

Region 3.

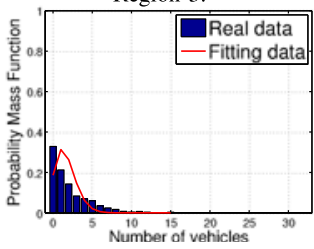

Region 8 .

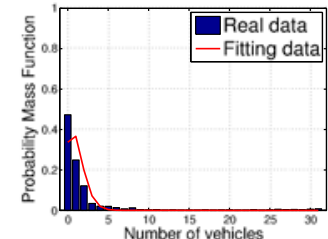

Region 4.

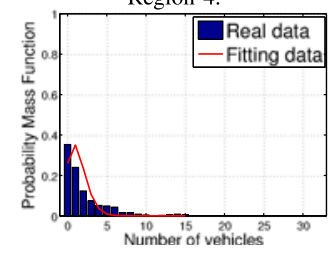

Region 9.

Fig. 3. Taxis position distributions of different regions at Beijing.

specific time instant are illustrated. We can see that the vehicles' position distribution reflects the planning structure of Beijing. Furthermore, we can distinguish the downtown and suburban areas. For the sake of illustrating the specific regional characteristics, instead of painting a picture of the whole city, we separate Beijing city into nine regions, as shown in Fig. 2. Based on the taxi-location information, we can estimate the intensity of vehicles in the different regions, as shown in Table I, where Region 0 represents Beijing city as a whole. The estimation process is subdivided into the following two steps: 1) We first calculate and store the number of taxis within a circle having a radius of $60 \mathrm{~m}$, which constitute a series of samples assumed to obey the Poisson distribution; and 2) then, we estimate the intensity $\lambda$ according to the distribution in (1) by using the maximum likelihood method. Moreover, we run the Kolmogorov-Smirnov test (K-S test) to verify that the real data indeed satisfies the PPP. In Table II, we show the K-S test output for each region, i.e., the $P$-value. Note that for $P \geq 0.05$, the hypothesis of exponential distribution is not denied. We can see that the $P$-values of all regions are higher than 0.05 , i.e., the taxi location data indeed satisfies the PPP.

Fig. 3 shows the c.d.f. of the number of vehicles within a circle of $60 \mathrm{~m}$ radius in different regions, where the bars represent real sample data from the dataset and the curve is the fitted PPP c.d.f. As we assumed in the system model, the spatial distribution of the real-world vehicles may be deemed reasonably consistent with the PPP distribution characteristics. Furthermore, we can observe that Region 5 representing the central area of Beijing city exhibits the highest vehicle intensity shown in Table I, while Region 7 as a suburban area has a low vehicle intensity. Moreover, the average distance between two vehicles can also be obtained from the dataset, as shown in Table I. Note that since the dataset only contains the taxi locations of Beijing city, the distances between two vehicles appear to be relatively large. In the following simulations, we will apply a multiplier of 5 to those intensities seen in Table I under the assumption that there is one taxi among five vehicles.

Based on the estimated intensity of vehicles, we can evaluate the information-sharing OP using the related parameters for the channel model listed in Table II, where the transmission power, the path loss, and fading models are configured according to [30]. Two typical scenarios are simulated: The first is the downtown scenario as in Region 1 of Beijing city, 607 where the signal channel between two peer vehicles should 608 obey the Nakagami- $m$ distribution, and the second is the 609 suburban scenario as in Region 7 of Beijing city, where the 610 channel obeys the Rayleigh distribution. For the downtown 611 scenario, we have to consider the effect of obstacles, such as 612 buildings. The influence of obstacles has been modeled in the 613 well-established simulators like Vergilius [40]-[42] or Veins 614 [43]-[45]. In this paper, we refer to the propagation model 615 introduced in Veins [43], where the obstacle effects $L_{\text {obs }}$ were 616 modeled by

$$
L_{\mathrm{obs}}[d B]=\beta_{w} n_{w}+\gamma_{w} d_{w}
$$

with $n_{w}$ representing the number of walls that the radio wave 618 has penetrated, $d_{w}$ represents the internal dimension of a 619 building, while $\beta_{w}$ and $\gamma_{w}$ represent a pair of calibration factors 620 having a value of $9.2 \mathrm{~dB}$ per wall and $0.32 \mathrm{~dB}$ per meter [43], 621 respectively. The building-induced blocking mostly occurs near 622 the street intersections. Thus, we can assume the number of 623 wall penetration occurences between two vehicles to be two, 624 and the building's internal dimension to be $50 \mathrm{~m}$. In Beijing, 625 the average distance between two intersections is $2 \mathrm{~km}$, and 626 if we consider $50 \mathrm{~m}$ to be the blocked area, the percentage of 627 building blocking can be deemed 0.025 .

The estimated vehicle intensity parameters of Region 1 and Region 7 are multiplied by 5 in our simulations. Considering that the breakpoint-based path loss model is common and practical, we have simulated two path loss settings, i.e., $\alpha=2$ and 4 , which constitute a pair of common path loss parameters according to the experimental results of [30]. Thus, four cases are simulated in these two scenarios based on whether the channel's path loss is $\alpha=2$ or 4 and whether the SNR is 10 or $20 \mathrm{~dB}$, respectively. The simulations were conducted using MATLAB relying on the following procedure. The channel is first generated according to the fading distribution and to the large-scale path loss. Then, we calculate the expected probability of the SINR value being less than some threshold, given the fading and distance parameters.

Figs. 4 and 5 show the channel-induced OP of both the sub- 643 urban and downtown scenarios, where the simulation results 644 are all consistent with the theoretical results. In the downtown 645 


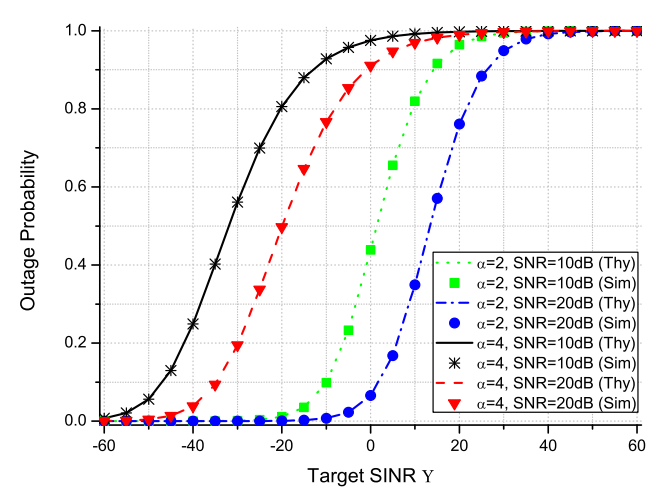

Fig. 4. Outage probability in Region 7.

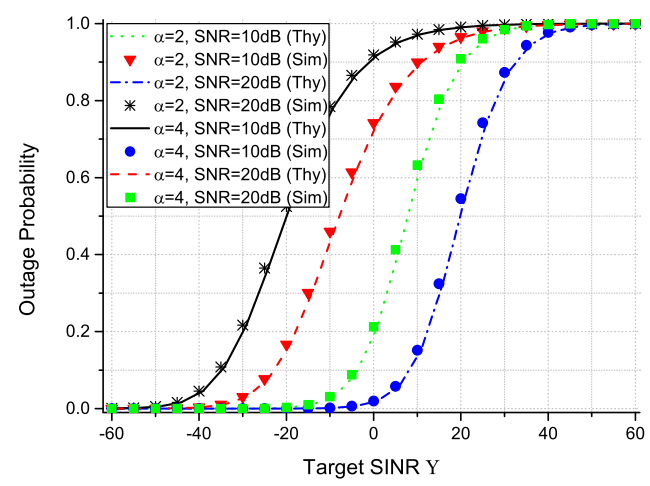

Fig. 5. Outage probability in Region 5 .

scenario, the simulation results are about $1 \mathrm{~dB}$ worse than the theoretical results, which is due to considering the buildinginduced blocking effects. The curves in those two figures are quite similar, which is expected due to having the same simulation settings. The only difference is that the channel-induced OP of the downtown scenario is lower than that in the suburban scenario owing to the reduced distance between a pair of vehicles, as well as due to having benign Nakagami fading channels. Generally, we can see that increasing the path loss exponent $\alpha$ from 2 to 4 can lead to the increase of channelinduced OP due to the higher power attenuation of the channel, while increasing the transmission power reduces the channelinduced OP. We also simulate the information-sharing OP of other regions of Beijing city, as shown in Fig. 6, where the path loss exponent is set to $\alpha=2$, the transmission SNR is set to $10 \mathrm{~dB}$, while the target received SINR is set to $\Upsilon=-10 \mathrm{~dB}$. We can see that the information-sharing OP is proportional to the intensity of vehicles in the region. This is because a low intensity implies a higher distance between two peer vehicles and the channel attenuation is more severe. Although the low vehicular intensity can also help reduce the interference imposed by other vehicles, this positive effect is dominated by the higher channel attenuation caused by the longer prorogation distance.

Based on the information-sharing OP, we can now conduct simulations to verify the benefits of our proposed RTI sharing mechanism. We invoke Algorithm 1 over 50 vehicles, where

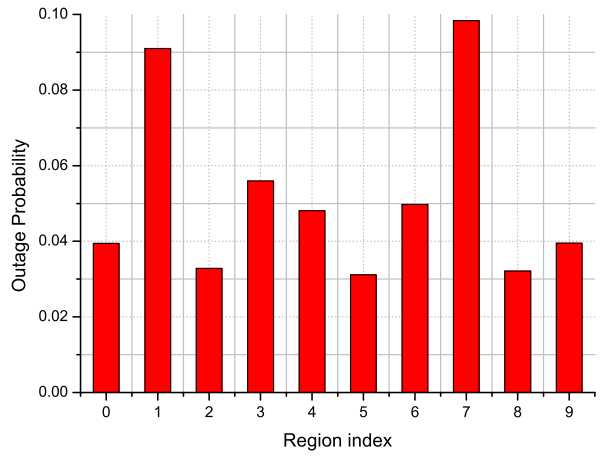

Fig. 6. Outage performance of all regions.

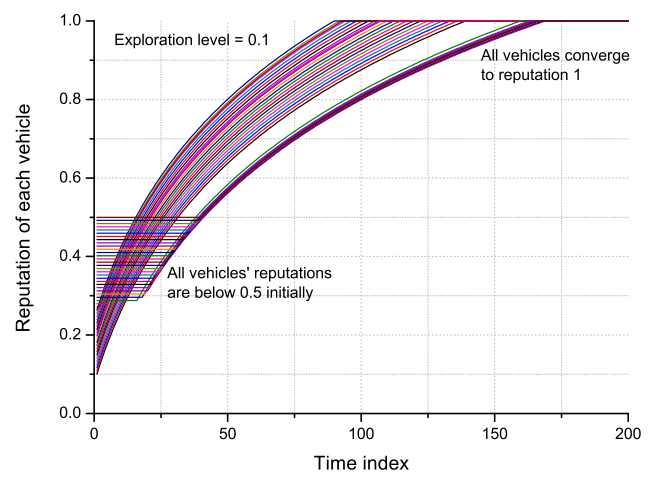

Fig. 7. Reputation of all vehicles $\xi=0.1$.

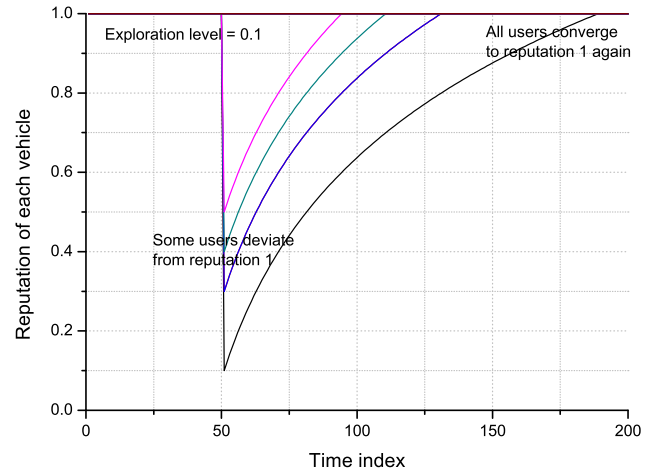

Fig. 8. Reputation of deviated vehicles $\xi=0.1$.

the reputation adjustment step size was configured according to 673 $\frac{0.02}{t}$ with $t$ being the time index. Fig. 7 shows the dynamics of 674 all vehicles' reputations during the learning and interaction pro- 675 cess, which also characterizes the vehicles' information-sharing 676 strategy. Although the vehicles are initially configured to have 677 different reputations below 0.5, i.e., to have a relatively low rep- 678 utation, the final converged all "1" reputation results corroborate 679 the high efficiency of our credit mechanism. To further verify the 680 stability of the proposed algorithm, we arrange for some vehicles 681 to deviate from the converged "1" reputation, as shown in Fig. 8. 682 It can be seen that all the vehicles that have deviated quickly con- 683 verged to reputation " 1 " again. Note, however, that the success 684 


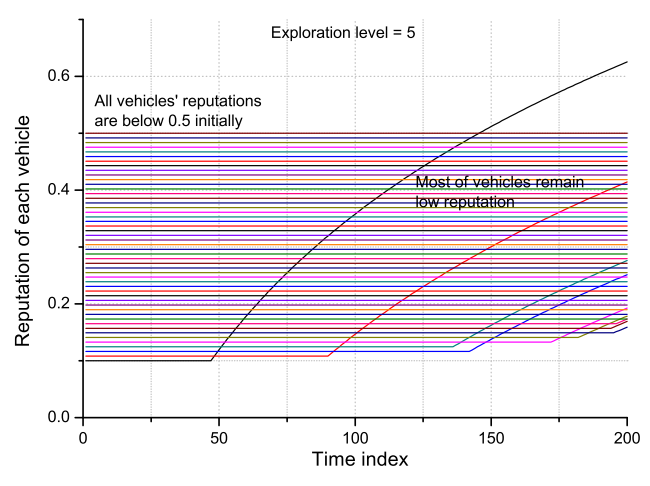

Fig. 9. Reputation of all vehicles $\xi=5$.

of convergence is conditioned on having an appropriate setting for the exploration level. An aggressive exploration may lead to divergence, as shown in Fig. 9, where the exploration level $\xi$ is set as high as 5. This is reasonable, because when the exploration level is excessive, the interaction probability tends to become binary according to (27), i.e., 0 or 1 . In such a case, some vehicles may not have the chance to interact with others and thus may not learn the reputation of others. Therefore, how to decide on a reasonable exploration level can be a promising future research topic.

\section{CONCLUSION}

In this paper, we studied the RTI sharing problem in vehicular networks, including both the theoretical channel-induced OP analysis and the genuine RTI sharing mechanism design. The theoretical analysis and the simulation results lead to the following major conclusions: 1) The outage performance is closely related to the density of vehicles, where a higher density implies having a reduced distance among the vehicles, which improves the communication performance; 2) the proposed credit-based RTI sharing mechanism is effective, which can ensure that all vehicles aspire to a good reputation, when an appropriate exploration level is adopted. Future research may include the theoretical information-sharing OP analysis under other vehicular network protocols, as well as genuine RTI sharing mechanism design relying on other kinds of incentives, instead of the credit considered here.

\section{APPENDIX A \\ PROOF OF THEOREM 1}

Following (10), we should calculate the expectation of $\mathbb{P}\left(\gamma_{0} \leq \Upsilon\right)$ with respect to vehicle $v_{1}$ 's location and channel, as well as all other $v_{i}$ 's locations and channels. First, let us take the expectations with respect to $d_{1}$. Since vehicle $v_{0}$ is sharing its RTI with the nearest vehicle $v_{1}$, no other vehicles can be closer than $d_{1}$, i.e., only vehicle $v_{0}$ is within the area $2 W d_{1}$. In this case, according to (1), the c.d.f. of $d_{1}$ can be formulated as follows:

$$
\begin{aligned}
& \mathbb{P}\left(d_{1} \leq D\right)=1-\mathbb{P}\left(d_{1}>D\right) \\
& =1-\mathbb{P}\left[\text { No other vehicle in } \pi D^{2} \mid \text { given the eixstence of } v_{0}\right] \\
& =1-e^{-2 \lambda W D}
\end{aligned}
$$

while the corresponding probability density function (p.d.f.) can 720 be written as

$$
f_{d_{1}}\left(d_{1}\right)=\frac{\mathrm{d}\left(1-e^{-2 \lambda W d_{1}}\right)}{\mathrm{d} d_{1}}=2 \lambda W e^{-2 \lambda W d_{1}} .
$$

In this case, the channel-induced OP of vehicle $v_{0}$ can be 722 expressed as

$$
\begin{aligned}
p_{0} & =1-\int_{d_{1}=0}^{+\infty} \mathbb{E}_{g_{1}, g_{i}, d_{i}}\left[\mathbb{P}\left(\gamma_{0}>\Upsilon\right)\right] f_{d_{1}}\left(d_{1}\right) \mathrm{d} d_{1} \\
& =1-\int_{d_{1}=0}^{+\infty} \mathbb{E}_{g_{1}, g_{i}, d_{i}}\left[\mathbb{P}\left(\frac{g_{1} d_{1}^{-\alpha_{1}}}{\Lambda}>\Upsilon\right)\right] 2 \lambda W e^{-2 \lambda W d_{1}} \mathrm{~d} d_{1} \\
& =1-2 \lambda W \int_{d_{1}=0}^{+\infty} \mathbb{E}_{g_{1}, g_{i}, d_{i}}\left[\mathbb{P}\left(g_{1}>d_{1}^{\alpha_{1}} \Upsilon \Lambda\right)\right] e^{-2 \lambda W d_{1}} \mathrm{~d} d_{1}
\end{aligned}
$$

Let us now concentrate our attention on the derivation of 724 $\mathbb{E}_{g_{1}, g_{i}, d_{i}}\left[\mathbb{P}\left(g_{1}>d_{1}^{\alpha_{1}} \Upsilon \Lambda\right)\right]$ shown in (32).

Since $g_{1}$ obeys the gamma distribution in (4), its c.d.f. can be 726 written as

$$
\begin{aligned}
F_{g_{1}}(X) & =\mathbb{P}\left[g_{1} \leq X\right]=1-\frac{\Gamma\left(m_{1}, \frac{m_{1}}{\mu_{1}} X\right)}{\Gamma\left(m_{1}\right)} \\
& =1-e^{-\frac{m_{1}}{\mu_{1}} X} \sum_{k=0}^{m_{1}-1} \frac{1}{k !} \frac{m_{1}^{k}}{\mu_{1}^{k}} X^{k}
\end{aligned}
$$

where $\Gamma(\cdot, \cdot)$ is the upper incomplete gamma function, $\mu_{1}$ is the 728 mean of $g_{1}$, and the last step is valid because we assume that the 729 Nakagami- $m$ fading parameter $m_{1}$ is an integer. ${ }^{1}$ In this case, 730 $\mathbb{E}_{g_{1}, g_{i}, d_{i}}\left[\mathbb{P}\left(g_{1}>d_{1}^{\alpha_{1}} \Upsilon \Lambda\right)\right]$ in (32) can be expressed as

$$
\begin{aligned}
& \mathbb{E}_{g_{1}, g_{i}, d_{i}}\left[\mathbb{P}\left(g_{1}>d_{1}^{\alpha_{1}} \Upsilon \Lambda\right)\right]=\mathbb{E}_{g_{i}, d_{i}} \\
& \times\left[\frac{\Gamma\left(m_{1}, \frac{m_{1}}{\mu_{1}} d_{1}^{\alpha_{1}} \Upsilon \Lambda\right)}{\Gamma\left(m_{1}\right)}\right] \\
& =\mathbb{E}_{\Lambda}\left[e^{-\frac{m_{1}}{\mu_{1}} d_{1}^{\alpha_{1}} \Upsilon \Lambda} \sum_{k=0}^{m_{1}-1} \frac{1}{k !} \frac{m_{1}^{k}}{\mu_{1}^{k}}\left(d_{1}^{\alpha_{1}} \Upsilon \Lambda\right)^{k}\right] \\
& =\int_{0}^{+\infty}\left[e^{-\frac{m_{1}}{\mu_{1}} d_{1}^{\alpha_{1}} \Upsilon \Lambda} \sum_{k=0}^{m_{1}-1} \frac{1}{k !} \frac{m_{1}^{k}}{\mu_{1}^{k}}\left(d_{1}^{\alpha_{1}} \Upsilon \Lambda\right)^{k}\right] f_{\Lambda}(\Lambda) \mathrm{d} \Lambda
\end{aligned}
$$

\footnotetext{
${ }^{1}$ When $m$ is an integer, we have the upper incomplete gama function $\Gamma(m, x)=(m-1) ! e^{-x} \sum_{k=0}^{m-1} \frac{x^{k}}{k !}$, the gamma function $\Gamma(m)=(m-1) !$, and $\frac{\Gamma(m, m x)}{\Gamma(m)}=e^{-m x} \sum_{k=0}^{m-1} \frac{m^{k}}{k !} x^{k}[46]$.
} 


$$
\begin{aligned}
& =\sum_{k=0}^{m_{1}-1} \frac{1}{k !}\left(\frac{m_{1} d_{1}^{\alpha_{1}} \Upsilon}{\mu_{1}}\right)^{k} \int_{0}^{+\infty}\left[e^{-\frac{m_{1} d_{1}^{\alpha}{ }^{1} \Upsilon}{\mu_{1}} \Lambda} \Lambda^{k}\right] f_{\Lambda}(\Lambda) \mathrm{d} \Lambda \\
& =\sum_{k=0}^{m_{1}-1} \frac{s^{k}}{k !}(-1)^{k} \frac{\mathrm{d}^{k} \mathcal{L}_{\Lambda}(s)}{\mathrm{d} s^{k}}
\end{aligned}
$$

733 where $f_{\Lambda}(\Lambda)$ represents the p.d.f. of $\Lambda$, and $s \triangleq \frac{m_{1} d_{1}^{\alpha}{ }_{1}^{1} \Upsilon}{\mu_{1}}$, and

$\mathcal{L}_{\Lambda}($.$) represents the Laplace transform of the interference plus$ noise of vehicle $v_{0}$, while the last step exploits the property of $x^{n} f(x) \stackrel{\mathcal{L}}{\Longleftrightarrow} \frac{\mathrm{d}^{k} \mathcal{L}_{\Lambda}(s)}{\mathrm{d}^{k}{ }^{k}}$.

The Laplace transform of $\Lambda$ can be calculated as follows:

$$
\begin{aligned}
\mathcal{L}_{\Lambda}(s) & =\mathbb{E}_{\Lambda}\left[e^{-s \Lambda}\right] \\
& =e^{-s \sigma^{2}} \mathbb{E}_{g_{i}, d_{i}}\left[\prod_{v_{i} \in \mathcal{S} \backslash\left\{v_{0}, v_{1}\right\}} e^{-s g_{i} d_{i}^{-\alpha}}\right] .
\end{aligned}
$$

Since all the vehicles $v_{i}\left(\forall v_{i} \in \mathcal{S} \backslash\left\{v_{0}, v_{1}\right\}\right)$ are independent of each other, all the channel gains $\left\{g_{i}\right\}$ are i.i.d. and their locations generated independently based on the PPP are also i.i.d.; hence, (35) can be rewritten as

$$
\begin{aligned}
\mathcal{L}_{\Lambda}(s) & =e^{-s \sigma^{2}} \mathbb{E}_{d_{i}}\left[\prod_{v_{i} \in \mathcal{S} \backslash\left\{v_{0}, v_{1}\right\}} \mathbb{E}_{g_{i}}\left[e^{-s g_{i} d_{i}^{-\alpha} 2}\right]\right] \\
& =e^{-s \sigma^{2}} \mathbb{E}_{d_{i}}\left[\prod_{v_{i} \in \mathcal{S} \backslash\left\{v_{0}, v_{1}\right\}} \frac{1}{1+s \mu_{2} d_{i}^{-\alpha_{2}}}\right] \\
& =e^{-s \sigma^{2}} \exp \left(-\lambda \int_{d_{1}}^{+\infty}\left(1-\frac{1}{1+s \mu_{2} \zeta^{-\alpha_{2}}}\right) 2 W \mathrm{~d} \zeta\right) \\
& =\exp \left(-s \sigma^{2}-2 \lambda W \int_{d_{1}}^{+\infty} \frac{1}{1+\frac{\zeta^{\alpha}}{\mu_{2} s}} \mathrm{~d} \zeta\right)
\end{aligned}
$$

where the second step is based on the assumption of experiencing a Rayleigh-fading channel with a mean of $\mu_{2}$ between vehicle $v_{i}$ (except for the closest vehicle $v_{1}$ ) and $v_{0}$. To elaborate a little further, the third step follows from the probability generating functional of the PPP [24] and the lower boundary of the integration is $d_{1}$, since the closest vehicle $v_{i}$ imposing interference on vehicle $v_{0}$ should be farther than $v_{0}$ 's peer vehicle $v_{1}$. By invoking the following change of variables $u=\frac{\zeta}{\left(\mu_{2} s\right)^{1 / \alpha_{2}}}$ in (36), we have

$$
\begin{aligned}
\mathcal{L}_{\Lambda}(s) & =\exp \left(-s \sigma^{2}-2 \lambda W\left(\mu_{2} s\right)^{1 / \alpha_{2}} \int_{\frac{d_{1}}{\left(\mu_{2}\right)^{1 / \alpha_{2}}}}^{+\infty} \frac{1}{1+u^{\alpha_{2}}} \mathrm{~d} u\right) \\
& =\exp \left[-s \sigma^{2}-2 \lambda W d_{1} \Phi_{\alpha_{2}}\left(\mu_{2} s d_{1}^{-\alpha_{2}}\right)\right]
\end{aligned}
$$

where $\Phi_{\alpha}(x)$ is as in (12). To summarize, by combining (32), (34), and (37), we arrive at vehicle $v_{0}$ 's channel-induced OP as

$$
\begin{aligned}
p_{0}= & 1-\left.2 \lambda W \int_{d_{1}=0}^{+\infty} \sum_{k=0}^{m_{1}-1} \frac{\left(-m_{1} d_{1}^{\alpha_{2}} \Upsilon\right)^{k}}{k ! \mu_{1}^{k}} \frac{\mathrm{d}^{k} \mathcal{L}_{\Lambda}(s)}{\mathrm{d} s^{k}}\right|_{s=\frac{m_{1} d_{1}^{\alpha_{1}} \Upsilon}{\mu_{1}}} \\
& e^{-2 \lambda W d_{1}} \mathrm{~d} d_{1}
\end{aligned}
$$

with $\mathcal{L}_{\Lambda}(s)$ in (37). By setting $d_{1}=\tau$, we have (11), which 753 completes the proof of Theorem 1.

\section{APPENDIX B}

PROOF OF COROLLARY 1

Since Rayleigh fading is a special case of Nakagami- $m 756$ fading associated with $m=1$, we can calculate vehicle $v_{0}$ 's 757 channel-induced OP in the highway scenario considered by 758 setting $m_{1}=1$ and $\mu_{1}=\mu_{2}=\mu$ in (11), which yields

$$
\begin{aligned}
p_{0} \mathrm{hwy}_{1}= & 1-2 \lambda W \int_{\tau=0}^{+\infty} \mathcal{L}_{\Lambda}\left(\frac{\tau^{\alpha_{1}} \Upsilon}{\mu}\right) e^{-2 \lambda W \tau} \mathrm{d} \tau \\
= & 1-2 \lambda W \int_{\tau=0}^{+\infty} \exp \\
& \times\left(-\frac{\tau^{\alpha_{1}} \Upsilon}{\mu} \sigma^{2}-2 \lambda W \int_{\tau}^{+\infty} \frac{1}{1+\frac{\zeta^{\alpha} 2}{\tau^{\alpha} 1}} \mathrm{~d} \zeta\right) \\
= & e^{-2 \lambda W \tau} \mathrm{d} \tau .
\end{aligned}
$$

By employing a change of variables $u=\frac{\zeta}{\tau^{\alpha_{1} / \alpha_{2}} \Upsilon^{1 / \alpha_{2}}}$, we can 760 rewrite (39) as

$$
\begin{aligned}
p_{0}^{\mathrm{hwy}_{1}}= & 1-2 \lambda W \int_{\tau=0}^{+\infty} e^{-2 \lambda W \tau-\frac{\tau^{\alpha} 1 \Upsilon}{\mu} \sigma^{2}-2 \lambda W \Upsilon^{\frac{1}{\alpha_{2}}} \tau^{\frac{\alpha_{1}}{\alpha_{2}}}} \\
& \times e^{\mathcal{G}_{\alpha_{2}}\left[\left(\frac{\tau^{\alpha_{2}-\alpha_{1}}}{\Upsilon}\right)^{\frac{1}{\alpha_{2}}}\right]} \mathrm{d} \tau \\
= & 1-2 \lambda W \int_{\tau=0}^{+\infty} \exp \left(-\frac{\sigma^{2} \Upsilon}{\mu} \tau^{\alpha_{1}}\right) \\
& \times \exp \left[-2 \lambda W \Upsilon^{\frac{1}{\alpha_{2}}} \tau^{\frac{\alpha_{1}}{\alpha_{2}}} \mathcal{G}_{\alpha_{2}}\left(\left(\frac{\tau^{\alpha_{2}-\alpha_{1}}}{\Upsilon}\right)^{\frac{1}{\alpha_{2}}}\right)\right] \\
& \cdot e^{-2 \lambda W \tau} \mathrm{d} \tau
\end{aligned}
$$

where according to [47], we have

$$
\begin{aligned}
\mathcal{G}_{\alpha}(x) & =\int_{x}^{+\infty} \frac{1}{1+u^{\alpha}} \mathrm{d} u \\
& =\frac{1}{\alpha-1} \frac{x}{1+x^{\alpha}} \mathbf{F}\left(1,1 ; 2-\frac{1}{\alpha} ; \frac{1}{1+x^{\alpha}}\right)
\end{aligned}
$$

with the hypergeometric function given by $\mathbf{F}(a, b ; c ; z)=763$ $1+\sum_{n=1}^{+\infty} \frac{z^{n}}{n !} \prod_{m=0}^{n-1} \frac{(a+m)(b+m)}{c+m}$. Although (40) appears to 764 be complicated, its physical interpretation is quite clear. The 765 first term $\exp \left(-\frac{\sigma^{2} \Upsilon}{\mu} \tau^{\alpha_{1}}\right)$ within the integration represents the 766 channel-induced OP as a function of noise, the second term 767 $\exp \left[-2 \lambda W \Upsilon^{\frac{1}{\alpha_{2}}} \tau^{\frac{\alpha_{1}}{\alpha_{2}}} \mathcal{G}_{\alpha_{2}}\left(\left(\frac{\tau^{\alpha_{2}-\alpha_{1}}}{\gamma}\right)^{\frac{1}{\alpha_{2}}}\right)\right]$ represents the channel- 768 induced OP influenced by the other vehicles $v_{i}$, and the last 769 term $e^{-2 \lambda W \tau}$ is associated with the p.d.f. of the variable $\tau=d_{1} . \quad 770$ This completes the proof of Corollary 1. 
APPENDIX C

PROOF OF COROLLARY 2

By substituting $\alpha_{1}=\alpha_{2}=\alpha$ in (13), we have

$$
\begin{aligned}
p_{0}^{\mathrm{hwy}_{2}=} & 1-2 \lambda W \int_{\tau=0}^{+\infty} \exp \left(-\frac{\sigma^{2} \Upsilon}{\mu} \tau^{\alpha}\right) \\
& \times \exp \left[-2 \lambda W \Upsilon^{\frac{1}{\alpha}} \mathcal{G}_{\alpha}\left(\Upsilon^{-\frac{1}{\alpha}}\right) \tau\right] \cdot e^{-2 \lambda W \tau} \mathrm{d} \tau \\
= & 1-2 \lambda W \int_{\tau=0}^{+\infty} \exp \left(-\frac{\sigma^{2} \Upsilon}{\mu} \tau^{\alpha}\right) \\
& \times \exp \left(-2 \lambda W \Phi_{\alpha}(\Upsilon) \tau\right) \cdot e^{-2 \lambda W \tau} \mathrm{d} \tau \\
= & 1-2 \lambda W \int_{\tau=0}^{+\infty} \exp \\
& \times\left[-\frac{\sigma^{2} \Upsilon}{\mu} \tau^{\alpha}-2 \lambda W\left(1+\Phi_{\alpha}(\Upsilon)\right) \tau\right] \mathrm{d} \tau
\end{aligned}
$$

where the second step is valid according to (12)

$$
\Phi_{\alpha}(\Upsilon)=\Upsilon^{1 / \alpha} \int_{\Upsilon-1 / \alpha}^{+\infty} \frac{1}{1+u^{\alpha}} \mathrm{d} u=\Upsilon^{1 / \alpha} \mathcal{G}_{\alpha}\left(\Upsilon^{-1 / \alpha}\right)
$$

$$
\begin{aligned}
p_{0}^{\mathrm{hwy}_{2}=} & 1-2 \lambda W \int_{\tau=0}^{+\infty} \exp \left(-\frac{\sigma^{2} \Upsilon}{\mu} \tau^{2}\right) \\
& \times \exp \left(-2 \lambda W \Upsilon^{\frac{1}{2}} \mathcal{G}_{2}\left(\Upsilon^{-\frac{1}{2}}\right) \tau\right) \cdot e^{-2 \lambda W \tau} \mathrm{d} \tau \\
= & 1-2 \lambda W \int_{\tau=0}^{+\infty} \exp \\
& \times\left(-\frac{\sigma^{2} \Upsilon}{\mu} \tau^{2}-2 \lambda W(1+\sqrt{\Upsilon} \arctan \sqrt{\Upsilon}) \tau\right) \mathrm{d} \tau \\
= & 1-2 \lambda W \sqrt{\frac{\pi}{\chi_{1}(\Upsilon)}} \exp \left(\frac{\chi_{2}^{2}(\Upsilon)}{4 \chi_{1}(\Upsilon)}\right) \\
& \times Q\left(\frac{\chi_{2}(\Upsilon)}{\sqrt{2 \chi_{1}(\Upsilon)}}\right)
\end{aligned}
$$

780 where the second step is valid because $\arctan (1 / u)=$ $781 \int_{u}^{+\infty} \frac{1}{1+u^{2}} \mathrm{~d} u$ and the last step exploits the following exponential 782 integration properties [46]:

$$
\int_{\tau=0}^{+\infty} \exp \left(-a \tau^{2}-b \tau\right) \mathrm{d} \tau=\sqrt{\frac{\pi}{a}} \exp \left(\frac{b^{2}}{4 a}\right) Q\left(\frac{b}{\sqrt{2 a}}\right)
$$

783 with the $Q$-function given by $Q(x)=\frac{1}{2 \pi} \int_{x}^{+\infty} \exp \left(-y^{2} / 2\right) \mathrm{d} y$. 784

This completes the proof of Corollary 2 .

\section{REFERENCES}

[1] S. Panichpapiboon and W. Pattara-Atikom, "A review of information dissemination protocols for vehicular ad hoc networks," IEEE Commun. Surveys Tuts., vol. 14, no. 3, pp. 784-798, Mar. 2012.

[2] D. Williams, "The arbitron national in-car study," [Online]. Available: http://www.arbitron.com /downloads/InCarStudy2009.pdf

[3] J. Zhao, Y. Zhang, and G. Cao, "Data pouring and buffering on the road: A new data dissemination paradigm for vehicular ad hoc networks," IEEE Trans. Veh. Technol., vol. 56, no. 6, pp. 3266-3277, Nov. 2007.

[4] J. Santa and A. Gomez-Skarmeta, "Sharing context-aware road and safety information," IEEE Pervasive Comput., vol. 8, no. 3, pp. 58-65, Jul. 2009.

[5] Y. Fallah, C.-L. Huang, R. Sengupta, and H. Krishnan, "Analysis of information dissemination in vehicular ad-hoc networks with application to cooperative vehicle safety systems," IEEE Trans. Veh. Technol., vol. 60, no. 1, pp. 233-247, Jan. 2011.

[6] N. Cenerario, T. Delot, and S. Ilarri, "A content-based dissemination protocol for vanets: Exploiting the encounter probability," IEEE Trans. Intell. Transp. Syst., vol. 12, no. 3, pp. 771-782, Sep. 2011.

[7] F. Ros, P. Ruiz, and I. Stojmenovic, "Acknowledgment-based broadcast protocol for reliable and efficient data dissemination in vehicular ad hoc networks," IEEE Trans. Mobile Comput., vol. 11, no. 1, pp. 33-46, Jan. 2012.

D. P. Garrido, F. Martinez, J. Cano, C. Calafate, and P. Manzoni, "An adaptive system based on roadmap profiling to enhance warning 808 message dissemination in vanets," IEEE/ACM Trans. Netw., vol. 21, no. 3, 809 pp. 883-895, Jun. 2013.

[9] N. Kumar and J-H. Lee, "Peer-to-peer cooperative caching for data dis- 811 semination in urban vehicular communications," IEEE Syst. J., vol. 8, 812 no. 4, pp. 1136-1144, Dec. 2014.

[10] J. Ahn, M. Sathiamoorthy, B. Krishnamachari, F. Bai, and L. Zhang, 814 "Optimizing content dissemination in vehicular networks with radio het- 815 erogeneity," IEEE Trans. Mobile Comput., vol. 13, no. 6, pp. 1312-1325, 816 Jun. 2014. message dissemination in vehicular ad hoc networks with priority," IEEE 819 J. Sel. Areas Commun., vol. 29, no. 1, pp. 61-71, Jan. 2011.

[12] Q. Wang, J. Hu, and J. Zhang, "Performance evaluation of information propagation in vehicular ad hoc network," IET Intell. Transport Syst., vol. 6, no. 2, pp. 187-196, Jun. 2012.

[13] K. Rostamzadeh and S. Gopalakrishnan, "Analysis of message dissemination in vehicular networks," IEEE Trans. Veh. Technol., vol. 62, no. 8, pp. 3974-3982, Oct. 2013.

[14] J. Hu, L.-L. Yang, and L. Hanzo, "Cooperative multicast aided picocellular hybrid information dissemination in mobile social networks: Delay/energy evaluation and relay selection," in Proc. IEEE Wirel. Commun. Netw. Conf., Istanbul, Turkey, Apr. 2014, pp. 3207-3212.

[15] J. Hu, L.-L. Yang, and L. Hanzo, "Throughput and delay analysis of wireless multicast in distributed mobile social networks based on geographic social relationships," in Proc. IEEE Wirel. Commun. Netw. Conf., Istanbul, Turkey, Apr. 2014, pp. 1874-1879. S. Dietzel, J. Petit, G. Heijenk, and F. Kargl, "Graph-based metrics for 835 insider attack detection in vanet multihop data dissemination protocols," 836

[17] K. Rostamzadeh, H. Nicanfar, N. Torabi, S. Gopalakrishnan, and V. Leung, 838 "A context-aware trust-based information dissemination framework for 839 vehicular networks," IEEE Internet Things J., vol. 2, no. 2, pp. 121-132, 840 Apr. 2015.

[18] M. Fogue et al., "Securing warning message dissemination in vanets using cooperative neighbor position verification," IEEE Trans. Veh. Technol., vol. 64, no. 6, pp. 2538-2550, Jun. 2015.

[19] T. Luan, R. Lu, X. Shen, and F. Bai, "Social on the road: Enabling secure and efficient social networking on highways," IEEE Wirel. Commun., vol. 22, no. 1, pp. 44-51, Feb. 2015.

[20] T. Luan, X. Shen, F. Bai, and L. Sun, "Feel bored? Join verse! Engineering 848 vehicular proximity social networks," IEEE Trans. Veh. Technol., vol. 64, 849 no. 3, pp. 1120-1131, Mar. 2015.

[21] J. Hu, L.-L. Yang, and L. Hanzo, "Distributed multistage cooperative- 851 social-multicast-aided content dissemination in random mobile networks," 852 IEEE Trans. Veh. Technol., vol. 64, no. 7, pp. 3075-3089, Jul. 2015.

[22] J. G. Andrews, F. Baccelli, and R. K. Ganti, "A tractable approach to 854 coverage and rate in cellular networks," IEEE Trans. Commun., vol. 59, 855 no. 11, pp. 3122-3134, Nov. 2011.

[23] H. Zhang, S. Chen, L. Feng, Y. Xie, and L. Hanzo, "A universal approach 857 to coverage probability and throughput analysis for cellular networks," 858 IEEE Trans. Veh. Technol., vol. 64, no. 9, pp. 4245-4256, Sep. 2015.
111] M. Khabazian, S. Aissa, and M. Mehmet-Ali "Performance modeling of IEEE Trans. Veh. Technol., vol. 62, no. 4, pp. 1505-1518, May 2013.

\section{5} 86
Q1 795 796 98 99 801
02 03 05 06 808 10 3 5 816
17 19
80 820 821 23 825 826 827 828 829
830 30 832 33 34 (n)

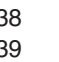
41

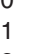

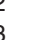

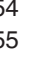
(t)

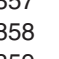
859 
[24] R. K. Ganti and M. Haenggi, "Interference and outage in clustered wireless ad hoc networks," IEEE Trans. Inf. Theory, vol. 35, no. 9, pp. 4067-4086, Sep. 2009.

[25] N. Alam, A. T. Balaei, and A. G. Dempster, "A DSRC doppler-based cooperative positioning enhancement for vehicular networks with gps availability," IEEE Trans. Veh. Technol., vol. 60, no. 9, pp. 4462-4470, Nov. 2011.

[26] P. Sujit, D. Lucani, and J. Sousa, "Bridging cooperative sensing and route planning of autonomous vehicles," IEEE Trans. Veh. Technol., vol. 30, no. 5, pp. 912-922, Jun. 2012.

[27] K. Xu, M. Gerla, and S. Bae, "How effective is the IEEE 802.11 RTS/CTS handshake in ad hoc networks," in Proc. IEEE Global Telecommun. Conf., Taipei, Taiwan, Nov. 2002, vol. 1, pp. 72-76.

[28] C. Jiang, H. Zhang, Y. Ren, and H. H. Chen, "Energy-efficient noncooperative cognitive radio networks: micro, meso, and macro views," IEEE Commun. Mag., vol. 52, no. 7, pp. 14-20, Jul. 2014.

[29] P. Gupta and P. Kumar, "The capacity of wireless networks," IEEE Trans. Inf. Theory, vol. 46, no. 2, pp. 388-404, Mar. 2000.

[30] L. Cheng, B. Henty, D. Stancil, F. Bai, and P. Mudalige, "Mobile vehicleto-vehicle narrow-band channel measurement and characterization of the $5.9 \mathrm{GHz}$ dedicated short range communication (DSRC) frequency band," IEEE J. Sel. Area Commun., vol. 25, no. 8, pp. 1501-1516, Oct. 2007.

[31] J. Hu, L.-L. Yang, and L. Hanzo, "Maximum average service rate and optimal queue scheduling of delay-constrained hybrid cognitive radio in Nakagami fading channels," IEEE Trans. Veh. Technol., vol. 62, no. 5, pp. 2220-2229, Jun. 2013.

[32] R. S. Sutton and A. G. Barto, Reinforcement Learning: An Introduction. Cambridge, MA, USA: MIT Press, 1998.

[33] M. Bennis, S. M. Perlaza, P. Blasco, Z. Han, and H. V. Poor, "Selforganization in small cell networks: A reinforcement learning approach," IEEE Trans. Wirel. Commun., vol. 12, no. 7, pp. 3202-3212, Jul. 2013.

[34] C. Jiang, Y. Chen, and K. J. R. Liu, "Multi-channel sensing and access game: Bayesian social learning with negative network externality," IEEE Trans. Wirel. Commun., vol. 13, no. 4, pp. 2176-2188, Apr. 2014.

[35] F. L. Lewis, D. Vrabie, and K. G. Vamvoudakis, "Reinforcement learning and feedback control: Using natural decision methods to design optimal adaptive controllers," IEEE Control Syst. Mag., vol. 32, no. 6, pp. 76-105, Dec. 2012.

[36] T. Matsui, T. Goto, K. Izumi, and Y. Chen, "Compound reinforcement learning: Theory and an application to finance," in Recent Advances in Reinforcement Learning (Lecture Notes in Computer Science). Berlin, Germany: Springer, 2012, pp. 321-332.

[37] R. M. Jones et al., "Behavioral and neural properties of social reinforcement learning," J. Neurosci., vol. 31, no. 37, pp. 13039-13045, Sep. 2011.

[38] C. Jiang, Y. Chen, Y. Gao, and K. J. R. Liu, "Indian buffet game with negative network externality and non-bayesian social learning," IEEE Trans. Syst., Man, Cybern., Syst., vol. 45, no. 4, pp. 609-623, Apr. 2015.

[39] J. Yuan et al., "T-drive: Driving directions based on taxi trajectories," in Proc. 18th SIGSPATIAL Int. Conf. Adv. Geographic Inf. Syst., Nov. 2010, pp. 99-108.

[40] E. Giordano, E. D. Sena, G. Pau, and M. Gerla, "Vergilius: A scenario generator for vanet," in IEEE 71st Veh. Technol. Conf., Taipei, Taiwan, May 2010, pp. 1-5.

[41] M. Cesana, L. Fratta, M. Gerla, E. Giordano, and G. Pau, "C-vet the ucla campus vehicular testbed: Integration of vanet and mesh networks," in Proc. Eur. Wirel. Conf., Lucca, Italy, Apr. 2014, pp. 689-695.

[42] E. Giordano, R. Frank, G. Pau, and M. Gerla, "Cooperative multicast aided picocellular hybrid information dissemination in mobile social networks: Delay/energy evaluation and relay selection," in Proc. 7th Int. Conf. Wirel. On-demand Netw. Syst. Serv., Kranjska Gora, Slovenia, Feb. 2010, pp. 57-60.

[43] C. Sommer, D. Eckhoff, and F. Dressler, "IVC in cities: Signal attenuation by buildings and how parked cars can improve the situation," IEEE Trans. Mobile Comput., vol. 13, no. 8, pp. 1733-1745, Aug. 2014.

[44] C. Sommer, R. German, and F. Dressler, "Bidirectionally coupled network and road traffic simulation for improved IVC analysis," IEEE Trans. Mobile Comput., vol. 10, no. 1, pp. 3-15, Jan. 2011.

[45] C. Sommer and F. Dressler, "Progressing toward realistic mobility models in VANET simulations," IEEE Commun. Mag., vol. 46, no. 11, pp. 132-137, Nov. 2008.

[46] I. S. Gradshteyn and I. M. Ryzhik, Table of Integrals, Series, Products. New York, NY, USA: Academic, 2007

[47] S. Mukherjee, "Distribution of downlink sinr in heterogeneous cellular networks," IEEE J. Sel. Area Commun., vol. 30, no. 3, pp. 575-585, Apr. 2012.

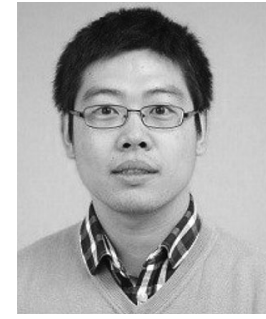

Chunxiao Jiang (S'09-M'13-SM'15) received the B.S. (Hons.) degree in information engineering from Beihang University, Beijing, China, in 2008 and the Ph.D. (Hons.) degree in electronic engineering from Tsinghua University, Beijing, in 2013

He is currently an Assistant Research Fellow with the Tsinghua Space Center, Tsinghua University. His research interests include the application of game theory, optimization, and statistical theories to communication, networking, signal processing, and resource allocation problems, in particular space information networks, heterogeneous networks, social networks, and big data privacy. He has authored/co-authored $100+$ technical papers in renowned international journals and conferences, including 50+ renowned IEEE journal papers.

Dr. Jiang has been actively involved in organizing and chairing sessions and has served as a member of the technical program committee, as well as the Symposium/Workshop Chair for a number of international conferences. He is currently an Editor of the Wiley Wireless Communications and Mobile Computing, Wiley Security and Communications Networks, the International Journa of Big Data Intelligence, and a Guest Editor of ACM/Springer Mobile Networks \& Applications Special Issue on "Game Theory for 5G Wireless Networks." He received the Best Paper Award from IEEE GLOBECOM in 2013, the Bes Student Paper Award from IEEE GlobalSIP in 2015, the Distinguished Dissertation Award from the Chinese Association for Artificial Intelligence (CAAI) in 2014, and the Tsinghua Outstanding Postdoc Fellow Award (only ten winners each year) in 2015 .

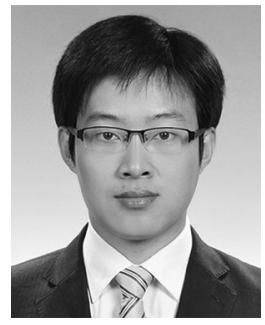

Haijun Zhang (M'13) received the Ph.D. degree from Beijing University of Posts Telecommunications (BUPT), Beijing, China.

$\mathrm{He}$ is a Postdoctoral Research Fellow with the Department of Electrical and Computer Engineering, University of British Columbia, Vancouver, BC, Canada. From September 2011 to September 2012, he visited the Centre for Telecommunications Research, King's College London, London, U.K., as a Visiting Research Associate, supported by the China Scholarship Council. He has published more than 70 papers and has authored two books. He serves as an Editor of Journal of Network and Computer Applications, Wireless Networks, Telecommunication Systems, and the KSII Transactions on Internet and Information Systems. He also has served as the Leading Guest Editor of ACM/Springer Mobile Networks \& Applications (MONET) Special Issue on "Game Theory for 5G Wireless Networks." He has served as the General Chair of GameNets'16, 5GWN2017, and the ICC2017 Workshop on 5GUDN and served as the Symposium Chair of the GameNets'14 and Track Chair of ScalCom'15. His current research interests include 5G, resource allocation, NOMA, LTE-U, heterogeneous small cell networks, and ultra-dense networks.

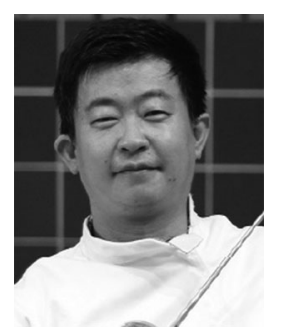

Zhu Han (S'01-M'04-SM'09-F'14) received the B.S. degree in electronic engineering from Tsinghua University, Beijing, China, in 1997 and the M.S. and Ph.D. degrees in electrical and computer engineering from the University of Maryland, College Park, MD, USA, in 1999 and 2003, respectively.

From 2000 to 2002, he was an R\&D Engineer with JDSU, Germantown, MD. From 2003 to 2006, he was a Research Associate with the University of Maryland. From 2006 to 2008, he was an Assistant Professor with Boise State University, Boise, ID, USA. $\mathrm{He}$ is currently a Professor with Electrical and Computer Engineering Department, as well as with the Computer Science Department, University of Houston, Houston, TX, USA. His research interests include wireless resource allocation and management, wireless communications and networking, game theory, big data analysis, security, and smart grid.

Dr. Han received the National Science Foundation Career Award in 2010, 1001 the Fred W. Ellersick Prize from the IEEE Communication Society in 2011, the 1002 EURASIP Best Paper Award for the Journal on Advances in Signal Processing 1003 in 2015, the IEEE Leonard G. Abraham Prize in the field of Communications 1004 Systems (best paper award in IEEE JSAC) in 2016, and several best paper 1005 awards at IEEE conferences. He is currently an IEEE Communications Society 1006 Distinguished Lecturer. ,

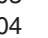

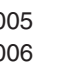




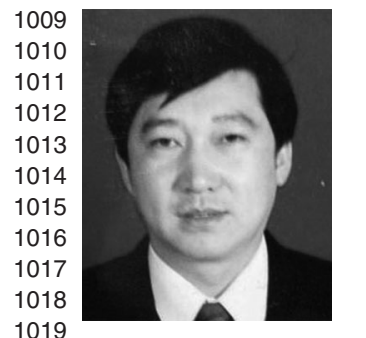

Yong Ren (SM'16) received the B.S., M.S., and Ph.D. degrees in electronic engineering from Harbin Institute of Technology, Harbin, China, in 1984, 1987, and 1994, respectively.

He was a Postdoctoral Researcher with the Department of Electronics Engineering, Tsinghua University, Beijing, China, from 1995 to 1997 . He is currently a Professor with the Department of Electronics Engineering and the Director of the Complexity Engineered Systems Lab, Tsinghua University. He holds 12 patents and has authored or co-authored 1020 more than 100 technical papers in the behavior of computer networks, peer-to1021 peer networks, and cognitive networks. His current research interests include 1022 complex systems theory and its applications to the optimization and information 1023 sharing of the Internet, Internet of things and ubiquitous networks, cognitive 1024 networks, and cyber-physical systems.

1025

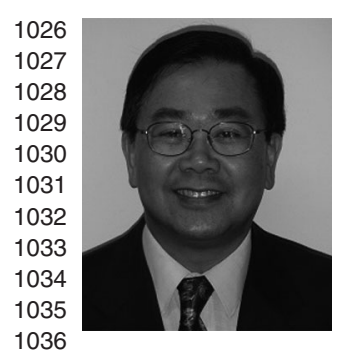

Victor C. M. Leung (S'75-M'89-SM'97-F'03) received the B.A.Sc. (Hons.) and Ph.D. degrees in electrical engineering from the University of British Columbia (UBC), Vancouver, BC, Canada, in 1977 and 1982, respectively. He received the Natural Sciences and Engineering Research Council Postgraduate Scholarship for his Ph.D. research.

From 1981 to 1987, he was a Senior Member of Technical Staff and satellite system specialist at MPR Teltech Ltd., Canada. In 1988, he was a Lecturer with the Department of Electronics, Chinese University of 1037 Hong Kong, Sha Tin, Hong Kong. He joined the UBC as a Faculty Member in 10381989 and is currently a Professor and the TELUS Mobility Research Chair of 1039 Advanced Telecommunications Engineering with the Department of Electrical 1040 and Computer Engineering. He has co-authored more than 900 technical papers 1041 in international journals and conference proceedings, 31 book chapters, and has 1042 co-edited 11 book titles. Several of his papers have been selected for best paper 1043 awards. His research interests include the areas wireless networks and mobile 1044 systems.

1045 Dr. Leung is a registered Professional Engineer in the Province of British 1046 Columbia, Canada. He is a Fellow of the Royal Society of Canada, the En1047 gineering Institute of Canada, and the Canadian Academy of Engineering. He 1048 was a Distinguished Lecturer of the IEEE Communications Society. He is a 1049 member of the editorial boards of IEEE WIRELESS COMMUNICATIONS LETTERS, 1050 the IEEE JOURNAL ON SELECTED AREAS IN COMMUNICATIONS Series on Green 1051 Communications and Networking, the IEEE TRANSACTIONS ON GREEN COM1052 MUNICATIONS AND NETWORKING, IEEE ACCESS, Computer Communications, 1053 and several other journals and has previously served on the editorial boards of 1054 the IEEE JOURNAL ON SELECTED AREAS IN COMMUNICATIONS-Wireless Com1055 munications Series, the IEEE TRANSACTIONS ON WIRELESS COMMUNICATIONS, 1056 the IEEE TRansactions on Vehicular Technology, the IEEE TRAnsaC1057 TIONS ON COMPUTERS, and the Journal of Communications and Networks. He 1058 has guest-edited many journal special issues and has provided leadership to the 1059 organizing committees and technical program committees of numerous con1060 ferences and workshops. He received the IEEE Vancouver Section Centennial 1061 Award and the 2012 UBC Killam Research Prize, as well as the APEBC Gold 1062 Medal as the head of the graduating class of the Faculty of Applied Science at 1063 UBC.

1064

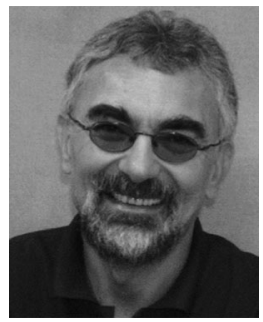

Lajos Hanzo (F'08) received the M.S. degree in elec- 1065 tronics and the Ph.D. degree from the Technical Uni- 1066 versity of Budapest, Budapest, Hungary, in 1976 and 1067 1983, respectively. He received the prestigious Doc- 1068 tor of Sciences research degree in wireless commu- 1069 nications from the University of Southampton, U.K., 1070 in 2004.

In 2016, he was admitted to the Hungarian 1072 Academy of Science, Budapest, Hungary. During his 1073 40-year career in telecommunications, he has held 1074 various research and academic posts in Hungary, 1075 Germany, and the U.K. Since 1986, he has been with the School of Electron- 1076 ics and Computer Science, University of Southampton, U.K., where he holds 1077 the Chair in telecommunications. He has successfully supervised $111 \mathrm{Ph} . \mathrm{D} .1078$ students, coauthored 20 John Wiley/IEEE Press books on mobile radio com- 1079 munications, totalling in excess of 10000 pages, published $1600+$ research 1080 contributions on IEEE Xplore, acted both as Technical Program Committee 1081 member and General Chair of IEEE conferences, presented keynote lectures, 1082 and received a number of distinctions. Currently he is directing a 60-strong 1083 academic research team, working on a range of research projects in the field 1084 of wireless multimedia communications sponsored by industry; the Engineer- 1085 ing and Physical Sciences Research Council (EPSRC), U.K.; and the European 1086 Research Council's Advanced Fellow Grant. He is an enthusiastic supporter of 1087 industrial and academic liaison, and he offers a range of industrial courses. He 1088 has $25000+$ citations and an $\mathrm{H}$-index of 60. For further information on research 1089 in progress and associated publications, see http://www-mobile.ecs.soton.ac.uk. 1090 Dr. Hanzo is also a Governor of the IEEE Vehicular Technology Society. Dur- 1091 ing 2008-2012, he was the Editor-in-Chief of the IEEE Press and a Chaired 1092 Professor with Tsinghua University, Beijing, China. In 2009, he received an 1093 honorary doctorate award by the Technical University of Budapest and in 2015, 1094 from the University of Edinburgh, Edinburgh, U.K., as well as the Royal Soci- 1095 ety's Wolfson Research Merit Award. He is a Fellow of the Royal Academy of 1096 Engineering, The Institution of Engineering and Technology, and EURASIP. 1097 
Q1. Author: Please provide missing year for Ref. [2]. 


\title{
Information-Sharing Outage-Probability Analysis of Vehicular Networks
}

\author{
Chunxiao Jiang, Senior Member, IEEE, Haijun Zhang, Member, IEEE, Zhu Han, Fellow, IEEE, \\ Yong Ren, Senior Member, IEEE, Victor C. M. Leung, Fellow, IEEE, and Lajos Hanzo, Fellow, IEEE
}

\begin{abstract}
In vehicular networks, information dissemination/sharing among vehicles is of salient importance. Although diverse mechanisms have been proposed in the existing literature, the related information credibility issues have not been investigated. Against this background, in this paper, we propose a credible information-sharing mechanism capable of ensuring that the vehicles do share genuine road traffic information (RTI). We commence with the outage-probability analysis of information sharing in vehicular networks under both a general scenario and a specific highway scenario. Closed-form expressions are derived for both scenarios, given the specific channel settings. Based on the outage-probability expressions, we formulate the utility of RTI sharing and design an algorithm for promoting the sharing of genuine RTI. To verify our theoretical analysis and the proposed mechanism, we invoke a real-world dataset containing the locations of Beijing taxis to conduct our simulations. Explicitly, our simulation results show that the spatial distribution of the vehicles obeys a Poisson point process, and our proposed credible RTI sharing mechanism is capable of ensuring that all vehicles indeed do share genuine RTI with each other.
\end{abstract}

Index Terms-Credibility, information dissemination, information sharing, Poisson point process (PPP), reinforcement learning, vehicular networks.

V EHICULAR communications and their support networks were originally proposed for public safety applications and traffic efficiency enhancements, which necessitate reliable short-distance vehicle-to-vehicle and vehicle-to-infrastructure communications [1]. With the advent of advanced automobile technology, the globe's population has

Manuscript received September 4, 2015; revised February 3, 2016 and May 24, 2016; accepted September 26, 2016. Date of publication; date of current version. This work was supported in part by the National Natural Science Foundation China under Project 61371079 and Project 61471025 and in part by the U.S. National Science Foundation under Grant CPS-1646607, Grant ECCS1547201, Grant CCF-1456921, Grant CNS-1443917, Grant ECCS-1405121, and Grant NSFC61428101. The review of this paper was coordinated by the Editors of CVS TVT.

C. Jiang is with the Tsinghua Space Center, Tsinghua University, Beijing 100084, China (e-mail: jchx@tsinghua.edu.cn).

H. Zhang and V. C. M. Leung are with the Department of Electrical and Computer Engineering, The University of British Columbia, Vancouver, BC V6T 1Z4, Canada (e-mail: dr.haijun.zhang@ieee.org; vleung@ece.ubc.ca).

Z. Han is with the Department of Electrical and Computer Engineering and the Department of Computer Science, University of Houston, Houston, TX 77004 USA (e-mail: zhan2@uh.edu).

Y. Ren is with the Department of Electronic Engineering, Tsinghua University, Beijing 100084, China (e-mail: reny @tsinghua.edu.cn).

L. Hanzo is with the School of Electronic and Computer Science, University of Southampton, Southampton SO17 1BJ, U.K. (e-mail: 1h@ecs.soton.ac.uk).

Color versions of one or more of the figures in this paper are available online at http://ieeexplore.ieee.org.

Digital Object Identifier 10.1109/TVT.2016.2614369 become more mobile. For example, Americans ride 224 miles or more per week either as a driver or passenger, and the total time spent traveling in a vehicle per week is a staggering $18 \mathrm{~h}$ and $31 \mathrm{~min}$ [2]. Meanwhile, the vehicular users' demands for in-car communication have also been dramatically increasing, since a wealth of value-added services emerge such as safety message dissemination and in-car entertainment services.

Most of the existing works on information dissemination/sharing were focused on designing specific mechanisms, in particular scenarios of vehicular networks. However, the credibility of the shared road traffic information (RTI) has not been taken into account in those mechanisms. Although all the vehicles act in a cooperative manner, the selfish or malicious ones may share either random or manipulated information for the sake of attaining an unfair road priority. Hence, we consider this problem and propose a mechanism for ensuring that all vehicles share genuine RTI. Furthermore, we define the utility functions of vehicles in the RTI sharing mechanism for the sake of analyzing their incentives in the RTI sharing process, and provide a general analytical framework for the information-sharing outage probability (OP) of vehicular networks. The new contributions of this paper can be summarized as follows.

1) We derive the information-sharing $\mathrm{OP}$ of vehicular networks both for the general scenario modeled by Nakagami- $m$ fading and for a more specific highway scenario, where Rayleigh fading is considered.

2) In order to encourage vehicles to share genuine RTI, we design a mechanism based on the reinforcement learning model, where the concept of "reputation" is introduced for circumventing the vehicles" selfish behaviors by exploiting its similarity to human social networks.

3) The real-world dataset containing the locations of Beijing taxis is utilized for verifying the vehicles' spatial distribution characteristics. Based on the parameters inferred with the aid of training from this dataset, we verify our analytical outage performance results as well as the proposed mechanism by our real-world data-driven simulations.

The rest of the paper is organized as follows. We first summarize the related works in Section II. Then, our system model is introduced in Section III. Based on the system model, the information-sharing OP is derived both for the general Nakagami- $m$ as well as for the more specific Rayleighdistributed highway scenario in Sections IV and V, respectively. In Section VI, we present the proposed RTI sharing scheme, while Section VII provides our real-world data-driven simulation results. Finally, we conclude in Section VIII. 


\section{RELATED WORKS}

The provision of information dissemination/sharing among vehicles is of pivotal significance in vehicular networks, which has been extensively studied in the literature [3]-[21]. Specifically, Zhao etal. [3] proposed an architecture and analyzed the dissemination capacity, where the data emanating from the sources were buffered by vehicles and then it was rebroadcast at the intersections. Similarly, the concept of a "smart road" was introduced and an integrated vehicular system was conceived for the collection, management, and provision of context-aware information concerning the traffic density and driver location [4].

Later, the vehicular ad hoc network (VANET) concept was proposed for assisting the dissemination of critical vehicle tracking information [5]. Meanwhile, Cenerario et. al. designed an event-related information exchange/sharing protocol relying on a VANET in [6]. With the goal of supporting a wide range of vehicular networks, Ros et al. [7] proposed a broadcast algorithm relying on periodic beacon messages, which contained acknowledgments of the circulated broadcast messages. The urban scenario of vehicular networks was studied based on the road map information as prior knowledge in [8] and relying on peer-to-peer (P2P) cooperative caching in [9]. The heterogeneity of radio propagation was taken into account in [10], where the tradeoffs amongst parameters, such as the cost, delay, and optimized system utility, were analyzed. The performance analysis of information sharing in vehicular networks was carried out in [11]-[15]. More specifically, the distribution of concurrent transmissions was analyzed in [11], while the analysis of packet loss rate and packet transmission distance was provided in [12]. The analysis of end-to-end reliability was disseminated in [13], while the throughput and delay analysis was the subject of [14] and [15].

On the other hand, the security issues of vehicular information dissemination were investigated in [16]-[18]. Explicitly, a graph-based metric was proposed for insider attacker detection in [16], whilst a trustworthiness verification model was advocated in [17] and a cooperative neighbor position verification model was conceived in [18]. Moreover, the information sharing in vehicular networks was modeled by carefully adapting the perspective of social networks [19]-[21]. Most of the aforementioned contributions were focused on designing specific mechanisms for information dissemination/sharing in particular scenarios of vehicular networks. However, the credibility of the shared RTI has not been taken into account in those mechanisms, which hence inspired this paper.

\section{SYSTEM MODEL}

As illustrated in Fig. 1, we consider a cooperative vehicular network constituted by a group of vehicles denoted by $\mathcal{S}=\left\{v_{0}, v_{1}, v_{2}, \ldots, v_{i}, \ldots\right\}$. Since all the vehicles are independent of each other, although their locations are geographically constrained by the mesh of roads in a city, they can be viewed as being randomly distributed. By exploiting this property, we assume that the locations of the vehicles obey a Poisson point process (PPP) on the 2-D road mesh with an intensity of $\lambda$ (the number of vehicles per square kilometer). The PPP has been

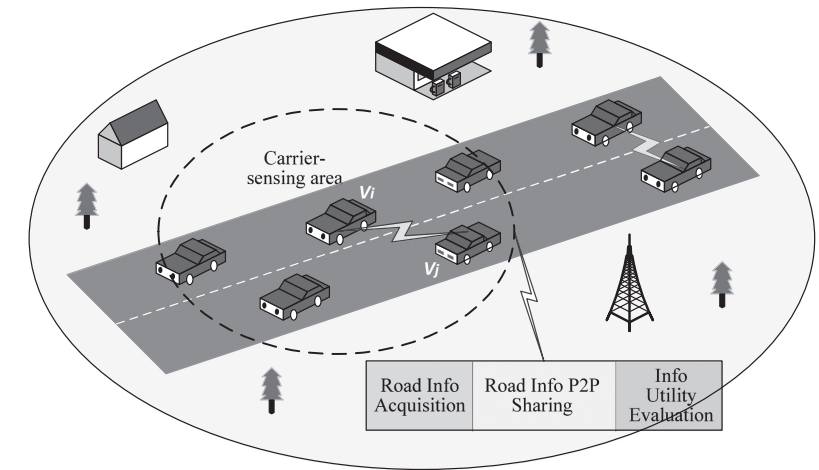

Fig. 1. System model.

widely adopted for modeling the distribution of random placements, such as the locations of macrocell and femtocell base stations [22], [23], as well as of ad hoc nodes [24]. In contrast to the existing PPP model of an infinite 2-D plane, the PPP model of a vehicular network is constrained by the road-width, which may nonetheless be as wide as say $100 \mathrm{~m}$ in metropolitan areas. Let us denote the road-width by $W$, which is assumed to be a constant. Based on the PPP model, the number of vehicles in any finite rectangle having a width of $W$ and a length of $D$ is Poisson distributed with a mean of $\lambda A_{r}$, which can be expressed as

$$
P\left(N_{r}=n\right)=\frac{e^{-\lambda W D}(\lambda W D)^{n}}{n !} .
$$

In our model, all the vehicles are assumed to be selfish, aiming for maximizing their own utility. We also assume that each vehicle has the capability of acquiring RTI and that they are willing to share it with each other in order to make better-informed decisions. The RTI can be for example the location information invoked for cooperative vehicle localization [25], or the traffic information invoked for cooperative route planning [26]. Our proposed model is general, and hence, it is independent of the specific form of the RTI. As shown in Fig. 1, at the beginning of each time slot, all the vehicles acquire the current RTI by their in-car sensors or by exploiting the driver's judgment. Then, each vehicle has to decide, whether it will truthfully share this information with others or whether to manipulate the shared RTI to render it useless, either, for example, due to privacy concerns or with the objective of gaining an unfair road priority. Therefore, although all the vehicles act in a cooperative manner, they occasionally may share random or manipulated information for the sake of improving their own utility. Then, each vehicle exchanges either its perceived genuine information or the false RTI with the nearest vehicle in a P2P mode. Following the information-sharing phase, each vehicle exploits its own information, as well as the shared information to make an informed decision as to whether to change speed, lanes, routes, or just maintain the current status. Finally, at the end of each time slot, the vehicle evaluates the performance attained as a result of its decision and then adjusts its actions in preparation for the next round. Here, we consider a practical scenario, where a vehicle is unable to ascertain the credibility of the RTI gleaned, until the 
information is actually utilized for its decision making and until the resultant performance is evaluated. Note that the time slot mentioned in this paper represents a coarse scale, on the order of seconds or minutes. Such a coarse synchronization can be readily achieved by the GPS, which has been widely deployed in vehicles. When it comes to information sharing between two vehicles, a fine-grained physical layer synchronization should be guaranteed for successful data transmission. However, such a fine-grained synchronization is not required for the entire network.

The above-mentioned P2P mode is assumed to be supported by the IEEE $802.11 \mathrm{p}$ protocol (a.k.a., the Wireless Access in the Vehicular Environment (WAVE)) relying on the classic Request To Send/Clear To Send (RTS/CTS) mechanism for the sake of avoiding the hidden terminal problem [27][28]. In this case, as shown in Fig. 1, only a single pair of vehicles is sharing information in a time slot within their carrier-sensing range, such as $v_{i}$ and $v_{j}$. Based on this characteristic, the two-directional outage analysis is not considered in this paper, since only a single pair of vehicles is engaged in communication within the range. Nevertheless, the vehicles beyond $v_{i}$ and $v_{j}$ 's carrier-sensing area may also impose interference on their communications according to the practical interference model of [29]. According to the experimental results of [30], the $5.9 \mathrm{GHz}$ dedicated short-range communications frequency band may be modeled by a Nakagami- $m$ fading channel, provided that the distance between two vehicles is below $40 \mathrm{~m}$. By contrast, it is modeled by a Rayleigh-fading channel when it is above $40 \mathrm{~m}$, which is a special case of the Nakagami- $m$ fading associated with $m=1$. A line-of-sight (LOS) Rician channel may also occur under certain circumstances. Nevertheless, we would like to concentrate on the Nakagami- $m$ and Rayleigh-fading scenarios, especially when it comes to the metropolitan areas, where the presence of buildings and of the infrastructure may block the LOS as in Beijing city. Thus, the power received by the vehicle $v_{i}$ from its peer $v_{j}$ located at a distance of $d_{i, j}$ can be expressed as

$$
y_{i, j}=\left|h_{i, j}\right|^{2} d_{i, j}^{-\alpha_{i, j}}
$$

where $\alpha_{i, j}$ is the channel's path loss coefficient and $h_{i, j}$ is the channel gain. Since the distance between a pair of communicating vehicles can be $40 \mathrm{~m}$ or higher, $h_{i, j}$ should obey the Nakagami- $m$ distribution of [31]:

$$
f_{h_{i, j}}(x)=2\left(\frac{m}{\mu_{i, j}}\right)^{m} \frac{x^{2 m-1}}{\Gamma(m)} \exp \left(-m \frac{x^{2}}{\mu_{i, j}}\right)
$$

where $\Gamma(\cdot)$ is the gamma function, $\mu_{i, j}=\mathbb{E}\left(\left|h_{i, j}\right|^{2}\right)$ is the average received power, and $m$ is the Nakagami- $m$ fading parameter. In this paper, we only consider integer $m$ values for the sake of mathematical tractability. Let us introduce $g_{i, j}=\left|h_{i, j}\right|^{2}$, where $g_{i, j}$ obeys the gamma distribution of

$$
f_{g_{i, j}}(x)=\left(\frac{m}{\mu_{i, j}}\right)^{m} \frac{x^{m-1}}{\Gamma(m)} \exp \left(-m \frac{x}{\mu_{i, j}}\right) .
$$

When using the IEEE $802.11 \mathrm{p}$ protocol, all the vehicles that impose interference on the vehicle $v_{i}$ in Fig. 1 should be located farther than $40 \mathrm{~m}$ [30]. In this case, the Rayleigh-fading model should be considered for the link imposing interference by the vehicle $v_{k}$ upon $v_{i}$, i.e., $g_{i, k}$ should obey the exponential distribution of

$$
f_{g_{i, k}}(x)=\frac{1}{\mu_{i, k}} \exp \left(-\frac{x}{\mu_{i, k}}\right) .
$$

\section{Channel-Induced Outage Probability in a General SCENARIO}

In this section, we theoretically analyze the channel-induced $\mathrm{OP}$ of vehicular networks. The classic channel-induced OP of a specific vehicle $v_{i}$ is defined as the probability of $v_{i}$ 's signal-tointerference-plus-noise ratio (SINR) dipping below a threshold of $\Upsilon$, i.e.,

$$
p_{v_{i}}=\mathbb{P}\left[\gamma_{v_{i}} \leq \Upsilon\right]
$$

which, in fact, is also the cumulative distribution function (c.d.f) 234 of this vehicle's SINR. Since the channel-induced OP is a 235 physical-layer metric, the fact of whether a vehicle shares gen- 236 uine or false information is irrelevant in this section. By contrast, 237 in Section V, we will use the channel-induced OP for modeling 238 the vehicles' future utility trend, depending on whether they are 239 sharing genuine or false RTI.

As illustrated in the system model, we consider a P2P scenario, where every pair of closest vehicles exchange their respective RTI within each time slot. For a specific vehicle $v_{0}$, its closest counterpart $v_{1}$ should be the intended informationsharing peer. Let us denote the distance and channel gain of $v_{0}$ with respect to the transmitter of the vehicle $v_{1}$ by $d_{1}$ and $g_{1}$, respectively. Then, the SINR of the vehicle $v_{0}$ can be written as

$$
\gamma_{0}=\frac{g_{1} d_{1}^{-\alpha_{1}}}{\Lambda}
$$

where $\alpha_{1}$ is the path loss coefficient, and $\Lambda$ is the interference imposed by the other vehicles on the vehicle $v_{0}$ plus the noise power. Let us assume that $v_{1}$ is the vehicle closest to $v_{0}$. Then, according to the experimental results of [30], the channel gain $g_{1}$ should obey the gamma distribution as in (4) with a mean of $\mathbb{E}\left[g_{1}\right]=\mu_{1}$ and Nakagami- $m$ fading parameter of $m_{1}$. During the information sharing between the pair of vehicles $v_{0}$ and $v_{1}$, the signals of all other vehicles, represented by $v_{i}\left(\forall v_{i} \in\right.$ $\mathcal{S} \backslash\left\{v_{0}, v_{1}\right\}$ ), should be considered as interference. Let us denote the distance and channel gain between $v_{i}$ and $v_{0}$ by $d_{i}$ and $g_{i}$, respectively. In this case, the interference plus noise power $\Lambda$ can be calculated by

$$
\Lambda=\sum_{v_{i} \in \mathcal{S} \backslash\left\{v_{0}, v_{1}\right\}} g_{i} d_{i}^{-\alpha_{2}}+\sigma^{2}
$$

where $\alpha_{2}$ is the path loss coefficient and $\sigma^{2}$ is the variance of the zero-mean circularly symmetric complex-valued Gaussian noise. Assuming that the other vehicles-except for the closest one-are relatively far from $v_{0}$, Rayleigh fading prevails between $v_{i}$ and $v_{0}$, i.e., the interfering channel's gain $g_{i}$ obeys the exponential distribution as in (5). Since all vehicles are independent of each other, the channel gains $\left\{g_{i, v_{i} \in \mathcal{S} \backslash\left\{v_{0}, v_{1}\right\}}\right\}$ are independent identically distributed (i.i.d.), where $\mathbb{E}\left[g_{i, v_{i} \in \mathcal{S} \backslash\left\{v_{0}, v_{1}\right\}}\right]=\mu_{2}$. Thus, the SINR of 
vehicle $v_{0}$ becomes

$$
\gamma_{0}=\frac{g_{1} d_{1}^{-\alpha_{1}}}{\sum_{v_{i} \in \mathcal{S} \backslash\left\{v_{0}, v_{1}\right\}} g_{i} d_{i}^{-\alpha_{2}}+\sigma^{2}}
$$

where we have

$$
\mathcal{G}_{\alpha}(x)=\int_{x}^{+\infty} \frac{1}{1+u^{\alpha / 2}} \mathrm{~d} u .
$$

Proof: See the proof in Appendix A.

\section{INFORMATION-SHARING OUTAGE PERFORMANCE IN HIGHWAY SCENARIO}

In Theorem 1, (11) provides the information-sharing OP of vehicular networks in a general form, which can be used in any arbitrary scenario, including both dense and sparse vehicular network scenarios. However, when considering specific application scenarios, further approximations can be adopted in the derivation of Theorem 1 . In this section, we will consider a highway-specific scenario, where the distance amongst vehicles may be substantially higher than in the downtown area, say over $30 \mathrm{~m}$ on average. According to the experimental results of [30], the channel between a pair of vehicles in this highway scenario is Rayleigh fading, which implies that the channel between vehicle $v_{1}$ and $v_{0}$ is Rayleigh fading. Hence, $g_{1}$ in (7) obeys follow the exponential distribution with the same mean as $g_{i}$. In essence, this specific Rayleigh-fading highway scenario constitutes a special case of Nakagami- $m$ fading associated with $m=1$. The following corollary formulates the channel-induced $\mathrm{OP}$ in this highway scenario.

Corollary 1: In a highway vehicular network relying on the 802.11p protocol and RTS/CTS, a vehicle's information-sharing $\mathrm{OP}$ can be expressed as

$$
\begin{aligned}
& p_{0}^{\mathrm{hwy}_{1}}=1-2 \lambda W \int_{\tau=0}^{+\infty} \exp \left(-\frac{\sigma^{2} \Upsilon}{\mu} \tau^{\alpha_{1}}\right) \\
& \exp \left[-2 \lambda W \Upsilon^{\frac{1}{\alpha_{2}}} \tau^{\frac{\alpha_{1}}{\alpha_{2}}} \mathcal{G}_{\alpha_{2}}\left(\left(\frac{\tau^{\alpha_{2}-\alpha_{1}}}{\Upsilon}\right)^{\frac{1}{\alpha_{2}}}\right)\right] \cdot e^{-2 \lambda W \tau} \mathrm{d} \tau
\end{aligned}
$$

Proof: See the proof in Appendix B.

According to the experimental results of [30], in the highway scenario the path loss measurements showed a dual-slope model, having a break-point at the distance of $100 \mathrm{~m}$. When the distance between two vehicles is below $100 \mathrm{~m}$, the path loss coefficient is $\alpha$, while beyond $100 \mathrm{~m}$ it is $\beta$. Since $100 \mathrm{~m}$ is already at the limit of the 802.11p-based P2P information sharing, we can focus our attention on considering the scenario, where all vehicles' path loss models are identical, i.e., $\alpha_{1}=\alpha_{2}=\alpha$. Specifically, the experimental results of [30] showed that the path loss coefficient is $\alpha=2$ under $100 \mathrm{~m}$. The channel-induced OP of this specific scenario is formulated in the following corollary.

Corollary 2: In a highway vehicular network using the 802.11p protocol and RTS/CTS, where the path loss coefficients amongst the vehicles are identical, a vehicle's information-sharing OP can be expressed as

$$
\begin{aligned}
p_{0}^{\mathrm{hwy}}= & 1-2 \lambda W \int_{\tau=0}^{+\infty} \exp \\
& \times\left[-\frac{\sigma^{2} \Upsilon}{\mu} \tau^{\alpha}-2 \lambda W\left(1+\Phi_{\alpha}(\Upsilon)\right) \tau\right] \mathrm{d} \tau .
\end{aligned}
$$

Specifically, when the channel's path loss coefficient is $\alpha=2, \quad 318$ the closed-form expression of the channel-induced OP can be 319 formulated as

$$
\begin{aligned}
p_{0}^{\text {hwy }_{2}=} & 1-2 \lambda W \sqrt{\frac{\pi}{\chi_{1}(\Upsilon)}} \exp \left(\frac{\chi_{2}^{2}(\Upsilon)}{4 \chi_{1}(\Upsilon)}\right) \\
& \times Q\left(\frac{\chi_{2}(\Upsilon)}{\sqrt{2 \chi_{1}(\Upsilon)}}\right)
\end{aligned}
$$

where $\chi_{1}(\Upsilon)$ and $\chi_{2}(\Upsilon)$ are

$$
\begin{aligned}
& \chi_{1}(\Upsilon)=\frac{\sigma^{2}}{\mu} \Upsilon \\
& \chi_{2}(\Upsilon)=2 \lambda W(1+\sqrt{\Upsilon} \arctan \sqrt{\Upsilon}) .
\end{aligned}
$$

Proof: See the proof in Appendix C.

It can be seen that (16) gives a simple closed-form expression 323 for a single vehicle's information-sharing OP, which simply re- 324 lies on the calculation of the $Q$-function. If we now consider the 325 specific scenario, where the channel noise is negligible com- 326 pared to the interference arriving from the other vehicles $v_{i}, \quad 327$ i.e., for $\sigma^{2} / \Lambda \rightarrow 0$, the information-sharing OP can be further 328 simplified using the following corollary.

Corollary 3: In a highway vehicular network associated with the 802.11 p protocol and RTS/CTS, where the path loss coefficients of all vehicles are identical and the channel noise is negligible compared to the interference, a vehicle's informationsharing OP during a specific time slot can be expressed as

$$
p_{0}^{\mathrm{hwy}_{3}}=\frac{\Phi_{\alpha}(\Upsilon)}{1+\Phi_{\alpha}(\Upsilon)} .
$$

Specifically, when the channel's path loss coefficient is $\alpha=2, \quad 335$ we have

$$
p_{0}^{\mathrm{hwy}_{3}}=\frac{\sqrt{\Upsilon} \arctan \sqrt{\Upsilon}}{1+\sqrt{\Upsilon} \arctan \sqrt{\Upsilon}} .
$$


Proof: Equations (19) and (20) can be readily obtained by setting $\sigma^{2}=0$ in (15) and (16), respectively.

By now, we have completed the theoretical informationsharing OP analysis, which is an important metric that reflects whether information sharing can be reliably accomplished. Note that successful information sharing in the vehicular network relies both on successful transmission in the presence of no channel-induced outage and no genuineinformation-sharing outage. Based on the channel-induced OP analysis of this section, the next section will propose a RTI sharing mechanism that ensures for the vehicles to share genuine information.

\section{RoAD TRAFFic ENGINEERING SHARING MECHANISM}

In the previous section, we have studied the informationsharing OP of the vehicular network considered. Following the above performance analysis, this section will consider the vehicles' information-sharing strategies, utilities, and interactions during the RTI sharing process. Note that the sharing of RTI cannot succeed if a channel-induced outage happens between the vehicles. Let us consider a cooperative vehicular network supporting $N$ selfish vehicles indexed as $\left\{v_{1}, v_{2}, \ldots, v_{N}\right\}$, each aiming for maximizing its own utility. As mentioned in the introduction, although all vehicles share the RTI in a cooperative manner, their specific degree of altruism/selfishness determines whether they may share false or genuine RTI for the sake of improving their own utility by exploiting unfair priority on the road for example. Considering this issue, each vehicle $v_{i}$ is assumed to have a binary action space defined as follows:

$$
a_{i}= \begin{cases}\mathbf{S}_{G}: & \text { sharing genuine RTI } \\ \mathbf{S}_{F}: & \text { sharing false RTI. }\end{cases}
$$

As a counterpart, a mixed strategy can also be defined for vehicle $v_{i}$ in which $q_{i}$ represents the probability of vehicle $v_{i}$ sharing genuine RTI, complemented by a $\left(1-q_{i}\right)$ probability of false RTI. As mentioned in the system model, each vehicle evaluates the RTI gleaned from its peer vehicle at the end of each time slot. Additionally, we also consider a binary information reward space, where the genuine RTI earns a reward of $R$, while the issuance of false RTI results in a zero reward. In such a case, we can summarize vehicle $v_{i}$ 's utility functions as follows:

$$
\left\{\begin{array}{l}
U_{\mathrm{ij}}\left(\mathbf{S}_{G}, \mathbf{S}_{G}\right)=\left(1-p_{\mathrm{ij}}\right) R-c_{i} \\
U_{\mathrm{ij}}\left(\mathbf{S}_{G}, \mathbf{S}_{F}\right)=-c_{i} \\
U_{\mathrm{ij}}\left(\mathbf{S}_{F}, \mathbf{S}_{G}\right)=\left(1-p_{\mathrm{ij}}\right) R \\
U_{\mathrm{ij}}\left(\mathbf{S}_{F}, \mathbf{S}_{F}\right)=0
\end{array}\right.
$$

where $U_{\mathrm{ij}}(a, b)$ represents vehicle $v_{i}$ 's utility, when its strategy is $a$ and its peer $v_{j}$ 's strategy is $b$ with $p_{\mathrm{ij}}$ denoting the channelinduced OP between $v_{j}$ and $v_{i}$, and $c_{i}>0$ represents the additional cost of sharing genuine information. Then, $\left(1-p_{\mathrm{ij}}\right) R$ quantifies the expected reward. Additionally, it is assumed that the link's OP $p_{\mathrm{ij}}$ should be no higher than $1-\frac{c_{i}}{R}$; otherwise, no vehicle would share genuine RTI under any circumstances.

The credit mechanism of the vehicular networks considered may be designed by observing human social networks. The concept of "reputation" is rather important for everyone in the real world, where a person's credit/reputation is generated and updated according to his/her accumulated behaviors in human social networks. Explicitly, when interacting with a reputable person, we are inclined to maintain future contacts with him/her On the other hand, if we learned a lesson from interacting with someone having a bad reputation, a long-lasting cooperation may be unlikely. Similarly, in our cooperative vehicular network, each vehicle can evaluate the others' credit through learning from its interactions with other vehicles. In this case, a vehicle can determine whether to share its RTI with a specific vehicle according to that vehicle's credit/reputation. When a vehicle's credit is below a certain threshold, other vehicles would not share any RTI with it. It is expected that through rounds of interactions, each vehicle's credit can be gradually learned by the observations and evaluations of its shared RTI. According to this credit information, the vehicles associated with a low credit would obtain less and less shared RTI, and eventually they will have to change their RTI sharing strategy to improve their reputation. We assume that there is a central server and each vehicle can report its experience in sharing RTI with all others. As a result, the database records the vehicles' credit. The credit established by each vehicle is considered to be private information, which may not be appropriate for the server to release to the public. This is similar to our human social network, where the credit earned by each individual is not directly visible to others. Nevertheless, through rounds of interactions, one vehicle's credit can be gradually learned by others. Note that the central server is only used by the vehicles to inform the others about their RTI sharing experience and to store the credit value of each vehicle. Since the experience can be quantized to a low number of discrete levels, the amount of data related to each vehicle is relatively small. Therefore, the server does not have to maintain a large-scale database. A potential solution is that each vehicle stores its own experience and the credit values of other vehicles locally.

Similar to the human social networks, each vehicle of our vehicular network can have a credit value generated by its past behavior, and also determines its future behavior when sharing RTI with others. Let us define vehicle $v_{i}$ ' reputation value as $r_{i}$ in conjunction with $0 \leq r_{i} \leq 1$. Note that in human social networks, a person's behavior is typically consistent with his/her reputation, regardless of the specific credit of the other persons he/she is interacting with. Similarly, vehicle $v_{i}$ 's RTI sharing strategy $q_{i}$ should also be consistent with its reputation $r_{i}$, and thus these two parameters can be deemed to be identical, i.e., we have $r_{i}=q_{i}$. When $v_{i}$ has the knowledge of vehicle $v_{j}$ 's credit/reputation through rounds of RTI sharing interactions, $v_{i}$ can determine whether to cooperate with $v_{j}$ in the future. Let us define $v_{i}$ 's interaction probability and action with respect to other vehicles as

$$
\begin{aligned}
& \boldsymbol{\kappa}_{i}=\left[\kappa_{i 1}, \kappa_{i 2}, \ldots, \kappa_{\mathrm{iN}}\right] \\
& \boldsymbol{\eta}_{i}=\left[\eta_{i 1}, \eta_{i 2}, \ldots, \eta_{\mathrm{iN}}\right]
\end{aligned}
$$

where $0 \leq \kappa_{\mathrm{ij}} \leq 1$ represents $v_{i}$ 's probability of sharing RTI 434 with $v_{j}$, regardless whether this is genuine or false information, 435 
and $\eta_{\mathrm{ij}}=0$ or 1 represents whether or not to cooperate with $v_{j}$ in a specific time slot. In such a scenario, at the beginning of each time slot, each vehicle first has to determine its next action $\eta_{\mathrm{ij}}$, i.e., whether to cooperate with the nearest vehicle $v_{j}$, according to $v_{i}$ 's interaction probability $\kappa_{\mathrm{ij}}$. Then, if it has decided to share RTI with $v_{j}$, it has to further determine the RTI sharing action $a_{i}$, i.e., as to whether to share genuine or false RTI with a specific peer vehicle, according to both $v_{i}$ 's information-sharing strategy $q_{i}$ as well as to its reputation $r_{i}$.

Meanwhile, after rounds of RTI sharing interactions, vehicle $v_{i}$ should update its interaction probability $\boldsymbol{\kappa}_{i}$ according to its experience with the others or by querying the database. It is expected that through a series of alternating decision making and learning processes, the vehicles having a bad reputation would obtain decreasingly less shared RTI from the others, and thus they would have to ameliorate their credit/reputation by actively sharing genuine RTI hereafter.

During the multiround RTI sharing process, none of the vehicles has access to the other vehicles' information-sharing strategies, actions, and utilities. Moreover, due to the rapidly evolving topology of vehicular networks, each vehicle may share its RTI with different vehicles during different time slots. Hence, from an individual vehicle's perspective, the network including all other vehicles can be regarded as an external environment, within which the vehicle makes decisions and shares RTI with the goal of maximizing its own utility. Generally, each vehicle learns from its interactions with this dynamic environment and adapts to the environment by adjusting its strategies for the sake of gleaning an increased utility. Reinforcement learning is a powerful tool capable of solving such an adaptive environment-learning and decision-making problem [32]. Its actions are reminiscent of how an intelligent agent infers the unknown statistical features of its environment as well as its actions in the environment so as to maximize a certain notion of the cumulative reward, where the environment itself is gradually changed by the agent's actions. Reinforcement learning has been widely adopted in communications and networks [33], [34], control [35], finance, and economics [36], as well as in social science [37], [38].

In our model, one of the main technical problems is how each vehicle constructs its interaction probability vector $\boldsymbol{\kappa}_{i}$ after rounds of RTI sharing interactions with the others. Based on the reinforcement learning model, each vehicle should first construct its perception through learning the others' inclination in RTI sharing. The perception is a quantitative representation of the accumulated utilities, which records all the historical interactions of the past as well as the new interaction results. In other words, it relies on the exploitation of past knowledge and on the exploration of a new environment [32]. Let us define vehicle $v_{i}$ 's perception of the others' behaviors as $\mathbf{z}_{i}$, where

$$
\mathbf{z}_{i}=\left[z_{i 1}, z_{i 2}, \ldots, z_{\mathrm{iN}}\right]
$$

with $z_{\mathrm{ij}}$ being vehicle $v_{i}$ 's perception with respect to $v_{j}$. At the end of each time slot, $v_{i}$ first evaluates the utility of information received from $v_{j}$ and then utilizes this utility value for adjusting its perception associated with $v_{j}$, while keeping the perception of others unchanged, which can be expressed as

$$
z_{\mathrm{ij}}^{t+1}= \begin{cases}\left(1-\epsilon_{i}^{t}\right) z_{\mathrm{ij}}^{t}+\epsilon_{i}^{t} U_{\mathrm{ij}}^{t}, & \text { if } \eta_{\mathrm{ij}}^{t}=1 \\ z_{\mathrm{ij}}^{t}, & \text { if } \eta_{\mathrm{ij}}^{t}=0\end{cases}
$$

where the superscript $t$ represents the time slot, $U_{\mathrm{ij}}^{t}$ is $v_{i}$ 's utility 491 gleaned through exchanging information with $v_{j}$ during time 492 slot $t$, and $\epsilon_{i}^{t}$ is a sequence of averaging factors controlling the 493 rate of decay in conjunction with $\sum_{t} \epsilon_{i}^{t}=\infty$ and $\sum_{t}\left(\epsilon_{i}^{t}\right)^{2}<\infty . \quad 494$ The constraint of $\sum_{t} \epsilon^{t}=\infty$ is imposed for ensuring $\epsilon^{t}>0, \quad 495$ i.e., the new learned utility $U_{\mathrm{ij}}^{t}$ should always be incorporated. 496 By contrast, the constraint of $\sum_{t}\left(\epsilon^{t}\right)^{2}<\infty$ is used for ensuring 497 $\epsilon^{t}<1$, i.e., the history of the learned experience $z_{\mathrm{ij}}^{t}$ should 498 always be utilized.

After updating the perception $\mathbf{z}_{i}$, vehicle $v_{i}$ can utilize it for generating its interaction probability with respect to vehicle $v_{j}$. Apparently, the more utility $v_{i}$ can obtain through sharing RTI with vehicle $v_{j}$, the higher the interaction probability $\kappa_{\mathrm{ij}}$ should be, which represents a proportional relationship between $\kappa_{\mathrm{ij}}$ and $z_{\mathrm{ij}}$. Here, we adopt a normalized performance evaluation method based on the Boltzmann exploration rule formulated as follows [32]:

$$
\kappa_{\mathrm{ij}}^{t}=\frac{e^{\xi_{z}^{t} z_{\mathrm{ij}}^{t}}}{\max \left\{e^{\xi_{i}^{t} z_{\mathrm{ij}}^{t}}, \forall j\right\}}
$$

where the positive coefficient $\xi_{j}^{t}$ controls the exploration level 508 with $\xi_{j}^{t} \rightarrow 0$ leading to a 0.5 interaction probability, while for 509 $\xi_{j}^{t} \rightarrow \infty$ the action would concentrate only on one of the pure 510 unconditional cooperation or no cooperation strategy, whichever 511 results in a higher perception. The physical meaning of (27) is 512 that vehicle $v_{i}$ always shares RTI with that specific vehicle, 513 which can give $v_{i}$ the highest utility. Then, $v_{i}$ considers this 514 highest utility as a reference, when it determines its interaction 515 probability with others.

To summarize, the reinforcement learning-based credible RTI sharing scheme can be interpreted as a process, in which each vehicle learns about its utilities as well as perceptions, and then updates its estimation regarding the other vehicles' reputation as well as adjusts its interaction behavior accordingly using its accumulated perception. The evolution from $z_{\mathrm{ij}}^{t}$ to $z_{\mathrm{ij}}^{t+1}$ can be illustrated by a chain of iterative elementary steps: the initial perception gives rise to a random interaction probability that determines the interaction; by following the interaction and the information-sharing action, the resultant utility is evaluated and then the perception can be updated in the next round, and so on. The iterations can be simply expressed by the following illustrative chain:

$$
\begin{gathered}
z_{\mathrm{ij}}^{t} \rightarrow \kappa_{\mathrm{ij}}^{t} \rightarrow \eta_{\mathrm{ij}}^{t} \rightarrow U_{\mathrm{ij}}^{t} \rightarrow z_{\mathrm{ij}}^{t+1} \\
\downarrow \\
r_{i}^{t} \rightarrow q_{i}^{t} \rightarrow a_{i}^{t}
\end{gathered}
$$

where the arrow between $\kappa_{\mathrm{ij}}^{t}$ and $r_{i}^{t}$ means that when a vehicle 530 discovers that the number of other vehicles sharing RTI with it 531 is less than a certain threshold, the vehicle would consider to 532 increase its credit value in order to enhance its reputation by 533 


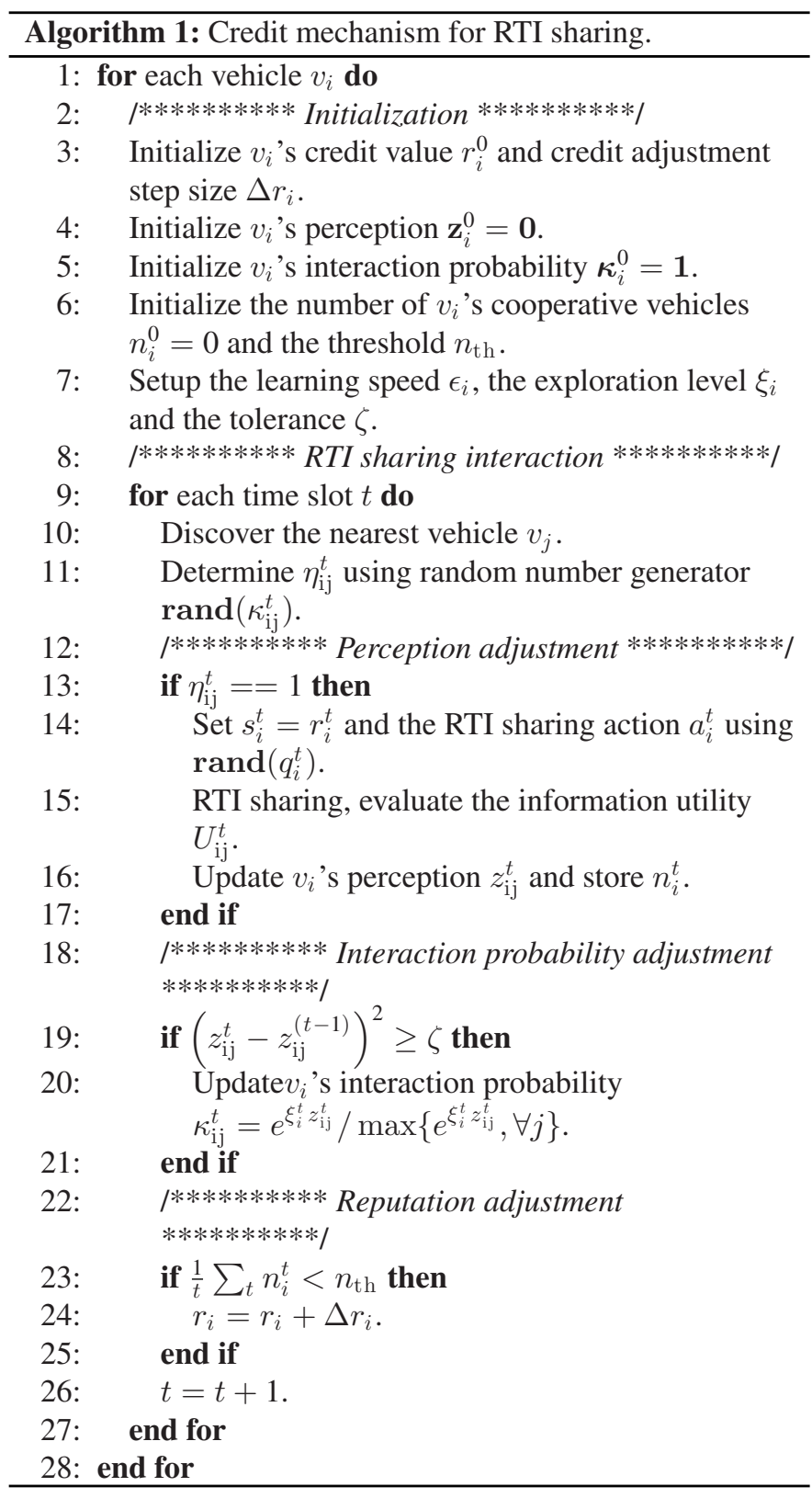

sharing more genuine RTI with the others. The credit mechanism is summarized in Algorithm 1. In the initialization phase, each vehicle may have different prior credit vales and credit adjustment preference. Meanwhile, the learning speed $\epsilon$ determines the weight of new information, the exploration level $\xi$ determines the probability of adopting uncharted strategies, while the tolerance determines the learning performance. In the RTI sharing phase, each vehicle first connects with the nearest vehicle and generates the interaction strategy, i.e., whether to interact with the vehicle. If the interaction indicator is positive, the vehicle then shares the genuine RTI with a probability generated by its reputation. Following the information-sharing interaction, the vehicle evaluates its perception and updates the interaction probability in the next round. If the vehicle finds that the number of other vehicles who would like to exchange information with it is below some threshold, the vehicle would

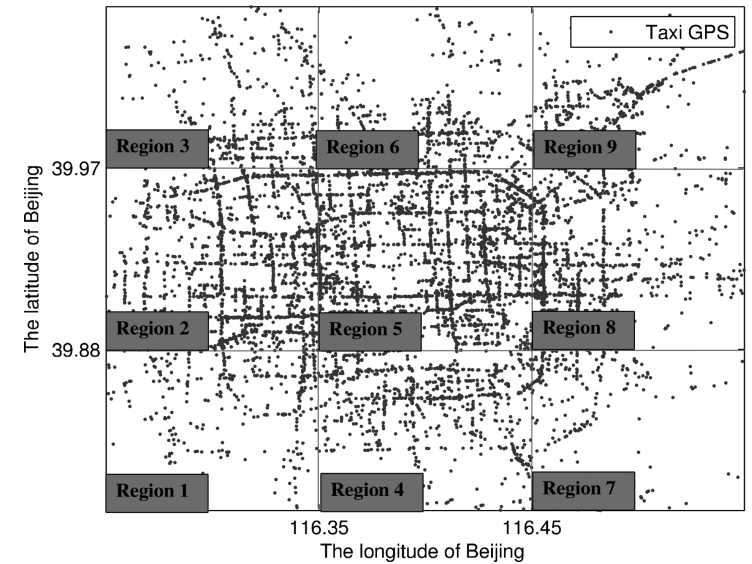

Fig. 2. Locations of Beijing taxis.

TABLE I

VEHICLE INTENSITIES OF DIFFERENT REGIONS AT BEIJING

\begin{tabular}{lccccc}
\hline \hline Region & 0 & 1 & 2 & 3 & 4 \\
\hline Intensity $\left(/ \mathrm{km}^{2}\right)$ & 59.6 & 23.3 & 72.7 & 40.7 & 48.1 \\
Average distance $(\mathrm{m})$ & 89.03 & 227.79 & 73.01 & 130.41 & 110.42 \\
K-S test $(P$-value) & 0.0731 & 0.1179 & 0.1061 & 0.0705 & 0.0619 \\
Region & 5 & 6 & 7 & 8 & 9 \\
Intensity $\left(/ \mathrm{km}^{2}\right)$ & 76.8 & 46.3 & 21.2 & 74.4 & 59.6 \\
Average distance $(\mathrm{m})$ & 69.12 & 114.57 & 250.00 & 71.35 & 89.05 \\
K-S test $(P$-value $)$ & 0.1169 & 0.0774 & 0.0831 & 0.0584 & 0.0937 \\
\hline \hline
\end{tabular}

TABLE II

Numerical Parameters For Performance Evaluation

\begin{tabular}{lc}
\hline \hline Parameter & Value \\
\hline Max Tx Power & $20 \mathrm{dBm}$ \\
Antennas & $1 \mathrm{Tx}, 1 \mathrm{Rx}$ \\
Antennas gains & $5 \mathrm{dBm}$ \\
Nakagami- $m$ fading parameter & $m=2$ \\
Path loss exponent & $\alpha=2,4$ \\
Noise power & $\sigma^{2}=0.1 \mathrm{dBm}$ \\
Maximum OP & $\Upsilon=0.1$ \\
\hline \hline
\end{tabular}

adjust its reputation according to the preferred adjustment step 550 size. In the next section, we will conduct simulations to quantify 551 the performance of the proposed algorithm.

\section{Simulation Results Based on Real Traffic Data 553}

In this section, we conduct simulations to verify our 554 theoretical analysis and characterize the proposed schemes. The 555 simulations are based on a real-world dataset consisting of the 556 spatial distribution of Beijing taxis. In the following, we will first 557 estimate the intensity of the taxis in Beijing using the dataset. 558 Then, based on the estimated intensity, we will characterize the 559 outage performance of RTI sharing as well as verify the merits 560 of the proposed RTI sharing scheme. 561

The real-world dataset contains the GPS positions of 10258562 taxis in Beijing (longitude from 116.25 to 116.55 and latitude 563 from 39.8 to 40.05) during the period of February 2-8, 2008564 [39]. As shown in Fig. 2, the positions of these vehicles at a 565 


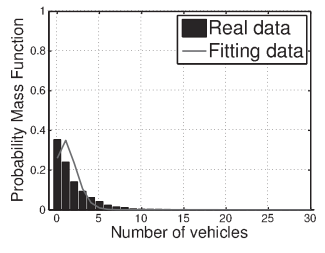

Whole Beijing.

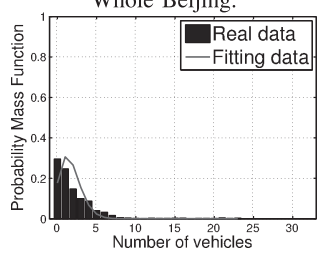

Region 5 .

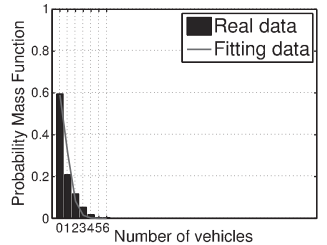

Region 1 .

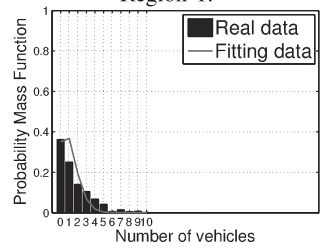

Region 6.

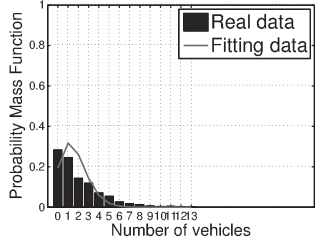

Region 2.

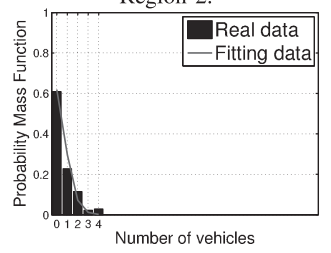

Region 7.

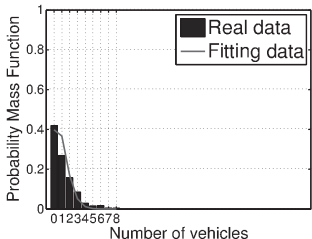

Region 3.

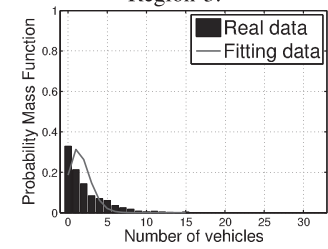

Region 8 .

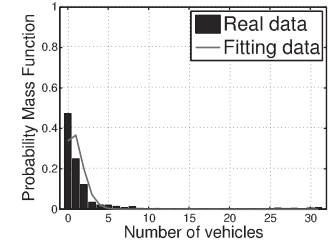

Region 4.

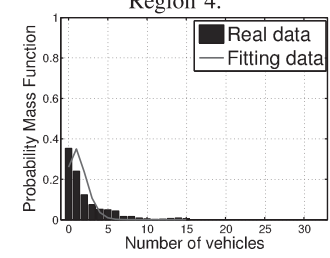

Region 9.

Fig. 3. Taxis position distributions of different regions at Beijing.

specific time instant are illustrated. We can see that the vehicles' position distribution reflects the planning structure of Beijing. Furthermore, we can distinguish the downtown and suburban areas. For the sake of illustrating the specific regional characteristics, instead of painting a picture of the whole city, we separate Beijing city into nine regions, as shown in Fig. 2. Based on the taxi-location information, we can estimate the intensity of vehicles in the different regions, as shown in Table I, where Region 0 represents Beijing city as a whole. The estimation process is subdivided into the following two steps: 1) We first calculate and store the number of taxis within a circle having a radius of $60 \mathrm{~m}$, which constitute a series of samples assumed to obey the Poisson distribution; and 2) then, we estimate the intensity $\lambda$ according to the distribution in (1) by using the maximum likelihood method. Moreover, we run the Kolmogorov-Smirnov test (K-S test) to verify that the real data indeed satisfies the PPP. In Table II, we show the K-S test output for each region, i.e., the $P$-value. Note that for $P \geq 0.05$, the hypothesis of exponential distribution is not denied. We can see that the $P$-values of all regions are higher than 0.05 , i.e., the taxi location data indeed satisfies the PPP.

Fig. 3 shows the c.d.f. of the number of vehicles within a circle of $60 \mathrm{~m}$ radius in different regions, where the bars represent real sample data from the dataset and the curve is the fitted PPP c.d.f. As we assumed in the system model, the spatial distribution of the real-world vehicles may be deemed reasonably consistent with the PPP distribution characteristics. Furthermore, we can observe that Region 5 representing the central area of Beijing city exhibits the highest vehicle intensity shown in Table I, while Region 7 as a suburban area has a low vehicle intensity. Moreover, the average distance between two vehicles can also be obtained from the dataset, as shown in Table I. Note that since the dataset only contains the taxi locations of Beijing city, the distances between two vehicles appear to be relatively large. In the following simulations, we will apply a multiplier of 5 to those intensities seen in Table I under the assumption that there is one taxi among five vehicles.

Based on the estimated intensity of vehicles, we can evaluate the information-sharing OP using the related parameters for the channel model listed in Table II, where the transmission power, the path loss, and fading models are configured according to [30]. Two typical scenarios are simulated: The first is the downtown scenario as in Region 1 of Beijing city, 607 where the signal channel between two peer vehicles should 608 obey the Nakagami- $m$ distribution, and the second is the 609 suburban scenario as in Region 7 of Beijing city, where the 610 channel obeys the Rayleigh distribution. For the downtown 611 scenario, we have to consider the effect of obstacles, such as 612 buildings. The influence of obstacles has been modeled in the 613 well-established simulators like Vergilius [40]-[42] or Veins 614 [43]-[45]. In this paper, we refer to the propagation model 615 introduced in Veins [43], where the obstacle effects $L_{\text {obs }}$ were 616 modeled by

$$
L_{\mathrm{obs}}[d B]=\beta_{w} n_{w}+\gamma_{w} d_{w}
$$

with $n_{w}$ representing the number of walls that the radio wave 618 has penetrated, $d_{w}$ represents the internal dimension of a 619 building, while $\beta_{w}$ and $\gamma_{w}$ represent a pair of calibration factors 620 having a value of $9.2 \mathrm{~dB}$ per wall and $0.32 \mathrm{~dB}$ per meter [43], 621 respectively. The building-induced blocking mostly occurs near 622 the street intersections. Thus, we can assume the number of 623 wall penetration occurences between two vehicles to be two, 624 and the building's internal dimension to be $50 \mathrm{~m}$. In Beijing, 625 the average distance between two intersections is $2 \mathrm{~km}$, and 626 if we consider $50 \mathrm{~m}$ to be the blocked area, the percentage of 627 building blocking can be deemed 0.025 .

The estimated vehicle intensity parameters of Region 1 and Region 7 are multiplied by 5 in our simulations. Considering that the breakpoint-based path loss model is common and practical, we have simulated two path loss settings, i.e., $\alpha=2$ and 4 , which constitute a pair of common path loss parameters according to the experimental results of [30]. Thus, four cases are simulated in these two scenarios based on whether the channel's path loss is $\alpha=2$ or 4 and whether the SNR is 10 or $20 \mathrm{~dB}$, respectively. The simulations were conducted using MATLAB relying on the following procedure. The channel is first generated according to the fading distribution and to the large-scale path loss. Then, we calculate the expected probability of the SINR value being less than some threshold, given the fading and distance parameters.

Figs. 4 and 5 show the channel-induced OP of both the sub- 643 urban and downtown scenarios, where the simulation results 644 are all consistent with the theoretical results. In the downtown 645 


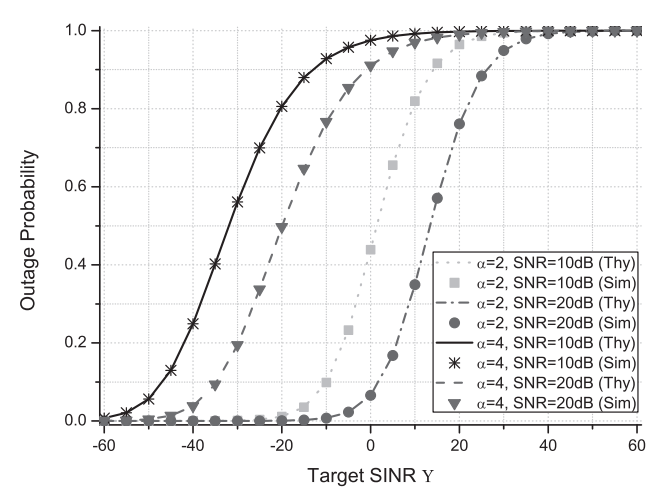

Fig. 4. Outage probability in Region 7.

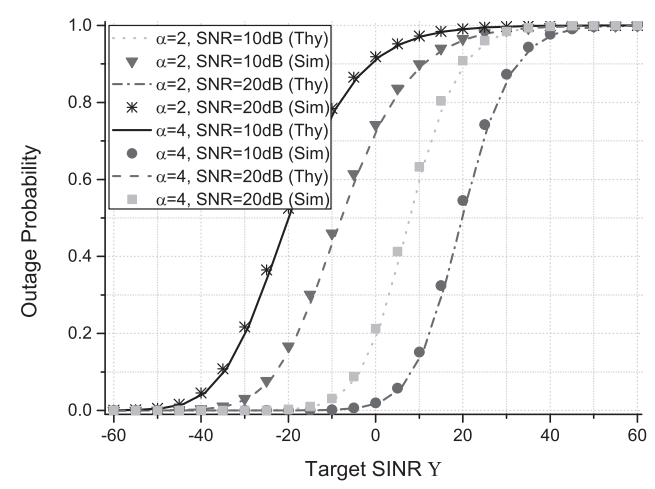

Fig. 5. Outage probability in Region 5.

scenario, the simulation results are about $1 \mathrm{~dB}$ worse than the theoretical results, which is due to considering the buildinginduced blocking effects. The curves in those two figures are quite similar, which is expected due to having the same simulation settings. The only difference is that the channel-induced OP of the downtown scenario is lower than that in the suburban scenario owing to the reduced distance between a pair of vehicles, as well as due to having benign Nakagami fading channels. Generally, we can see that increasing the path loss exponent $\alpha$ from 2 to 4 can lead to the increase of channelinduced OP due to the higher power attenuation of the channel, while increasing the transmission power reduces the channelinduced OP. We also simulate the information-sharing OP of other regions of Beijing city, as shown in Fig. 6, where the path loss exponent is set to $\alpha=2$, the transmission SNR is set to $10 \mathrm{~dB}$, while the target received SINR is set to $\Upsilon=-10 \mathrm{~dB}$. We can see that the information-sharing OP is proportional to the intensity of vehicles in the region. This is because a low intensity implies a higher distance between two peer vehicles and the channel attenuation is more severe. Although the low vehicular intensity can also help reduce the interference imposed by other vehicles, this positive effect is dominated by the higher channel attenuation caused by the longer prorogation distance.

Based on the information-sharing OP, we can now conduct simulations to verify the benefits of our proposed RTI sharing mechanism. We invoke Algorithm 1 over 50 vehicles, where

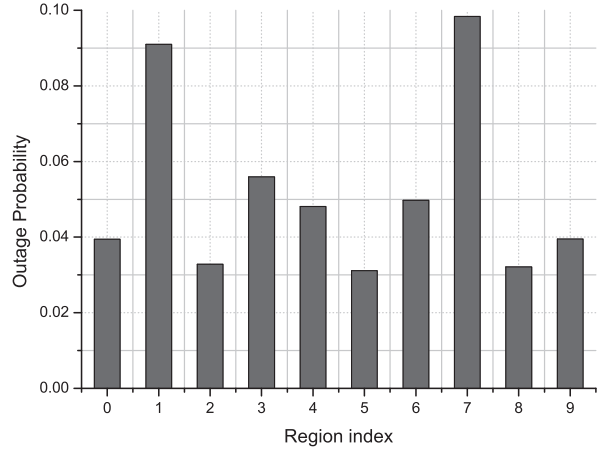

Fig. 6. Outage performance of all regions.

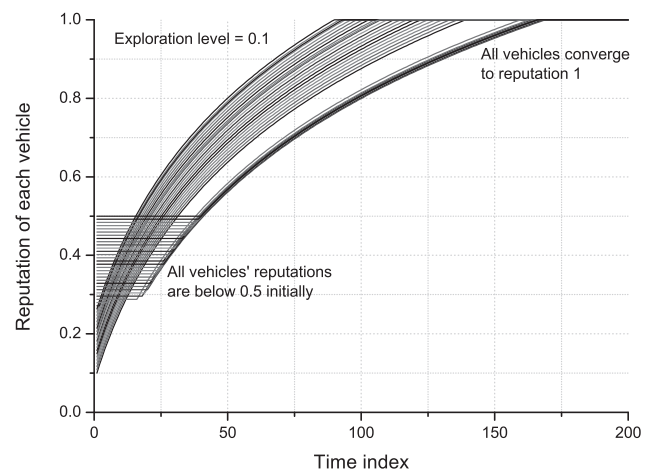

Fig. 7. Reputation of all vehicles $\xi=0.1$.

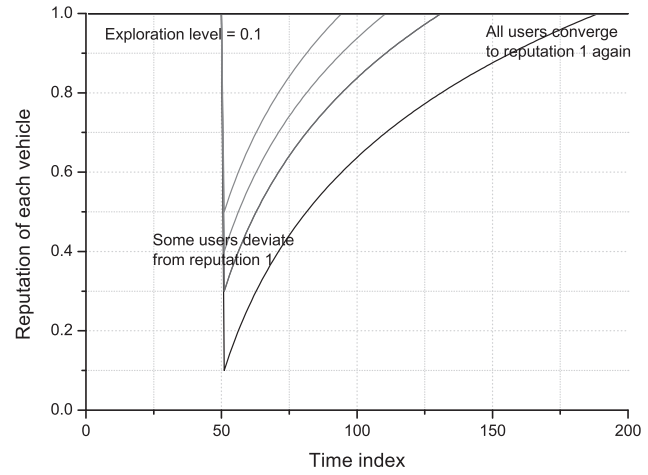

Fig. 8. Reputation of deviated vehicles $\xi=0.1$.

the reputation adjustment step size was configured according to 673 $\frac{0.02}{t}$ with $t$ being the time index. Fig. 7 shows the dynamics of 674 all vehicles' reputations during the learning and interaction pro- 675 cess, which also characterizes the vehicles' information-sharing 676 strategy. Although the vehicles are initially configured to have 677 different reputations below 0.5 , i.e., to have a relatively low rep- 678 utation, the final converged all "1" reputation results corroborate 679 the high efficiency of our credit mechanism. To further verify the 680 stability of the proposed algorithm, we arrange for some vehicles 681 to deviate from the converged "1" reputation, as shown in Fig. 8. 682 It can be seen that all the vehicles that have deviated quickly con- 683 verged to reputation " 1 " again. Note, however, that the success 684 


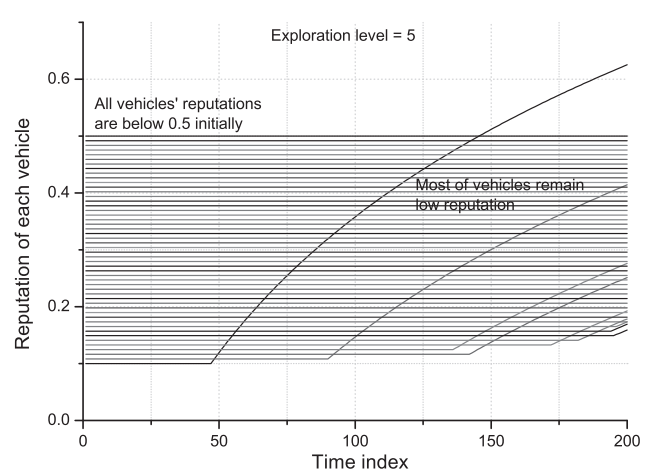

Fig. 9. Reputation of all vehicles $\xi=5$.

of convergence is conditioned on having an appropriate setting for the exploration level. An aggressive exploration may lead to divergence, as shown in Fig. 9, where the exploration level $\xi$ is set as high as 5. This is reasonable, because when the exploration level is excessive, the interaction probability tends to become binary according to (27), i.e., 0 or 1 . In such a case, some vehicles may not have the chance to interact with others and thus may not learn the reputation of others. Therefore, how to decide on a reasonable exploration level can be a promising future research topic.

\section{CONCLUSION}

In this paper, we studied the RTI sharing problem in vehicular networks, including both the theoretical channel-induced OP analysis and the genuine RTI sharing mechanism design. The theoretical analysis and the simulation results lead to the following major conclusions: 1) The outage performance is closely related to the density of vehicles, where a higher density implies having a reduced distance among the vehicles, which improves the communication performance; 2) the proposed credit-based RTI sharing mechanism is effective, which can ensure that all vehicles aspire to a good reputation, when an appropriate exploration level is adopted. Future research may include the theoretical information-sharing OP analysis under other vehicular network protocols, as well as genuine RTI sharing mechanism design relying on other kinds of incentives, instead of the credit considered here.

\section{APPENDIX A \\ PROOF OF THEOREM 1}

Following (10), we should calculate the expectation of $\mathbb{P}\left(\gamma_{0} \leq \Upsilon\right)$ with respect to vehicle $v_{1}$ 's location and channel, as well as all other $v_{i}$ 's locations and channels. First, let us take the expectations with respect to $d_{1}$. Since vehicle $v_{0}$ is sharing its RTI with the nearest vehicle $v_{1}$, no other vehicles can be closer than $d_{1}$, i.e., only vehicle $v_{0}$ is within the area $2 W d_{1}$. In this case, according to (1), the c.d.f. of $d_{1}$ can be formulated as follows:

$$
\begin{aligned}
& \mathbb{P}\left(d_{1} \leq D\right)=1-\mathbb{P}\left(d_{1}>D\right) \\
& =1-\mathbb{P}\left[\text { No other vehicle in } \pi D^{2} \mid \text { given the eixstence of } v_{0}\right] \\
& =1-e^{-2 \lambda W D}
\end{aligned}
$$

while the corresponding probability density function (p.d.f.) can 720 be written as

$$
f_{d_{1}}\left(d_{1}\right)=\frac{\mathrm{d}\left(1-e^{-2 \lambda W d_{1}}\right)}{\mathrm{d} d_{1}}=2 \lambda W e^{-2 \lambda W d_{1}} .
$$

In this case, the channel-induced OP of vehicle $v_{0}$ can be 722 expressed as

$$
\begin{aligned}
p_{0} & =1-\int_{d_{1}=0}^{+\infty} \mathbb{E}_{g_{1}, g_{i}, d_{i}}\left[\mathbb{P}\left(\gamma_{0}>\Upsilon\right)\right] f_{d_{1}}\left(d_{1}\right) \mathrm{d} d_{1} \\
& =1-\int_{d_{1}=0}^{+\infty} \mathbb{E}_{g_{1}, g_{i}, d_{i}}\left[\mathbb{P}\left(\frac{g_{1} d_{1}^{-\alpha_{1}}}{\Lambda}>\Upsilon\right)\right] 2 \lambda W e^{-2 \lambda W d_{1}} \mathrm{~d} d_{1} \\
& =1-2 \lambda W \int_{d_{1}=0}^{+\infty} \mathbb{E}_{g_{1}, g_{i}, d_{i}}\left[\mathbb{P}\left(g_{1}>d_{1}^{\alpha_{1}} \Upsilon \Lambda\right)\right] e^{-2 \lambda W d_{1}} \mathrm{~d} d_{1}
\end{aligned}
$$

Let us now concentrate our attention on the derivation of 724 $\mathbb{E}_{g_{1}, g_{i}, d_{i}}\left[\mathbb{P}\left(g_{1}>d_{1}^{\alpha_{1}} \Upsilon \Lambda\right)\right]$ shown in (32).

Since $g_{1}$ obeys the gamma distribution in (4), its c.d.f. can be 726 written as

$$
\begin{aligned}
F_{g_{1}}(X) & =\mathbb{P}\left[g_{1} \leq X\right]=1-\frac{\Gamma\left(m_{1}, \frac{m_{1}}{\mu_{1}} X\right)}{\Gamma\left(m_{1}\right)} \\
& =1-e^{-\frac{m_{1}}{\mu_{1}} X} \sum_{k=0}^{m_{1}-1} \frac{1}{k !} \frac{m_{1}^{k}}{\mu_{1}^{k}} X^{k}
\end{aligned}
$$

where $\Gamma(\cdot, \cdot)$ is the upper incomplete gamma function, $\mu_{1}$ is the 728 mean of $g_{1}$, and the last step is valid because we assume that the 729 Nakagami- $m$ fading parameter $m_{1}$ is an integer. ${ }^{1}$ In this case, 730 $\mathbb{E}_{g_{1}, g_{i}, d_{i}}\left[\mathbb{P}\left(g_{1}>d_{1}^{\alpha_{1}} \Upsilon \Lambda\right)\right]$ in (32) can be expressed as

$$
\begin{aligned}
& \mathbb{E}_{g_{1}, g_{i}, d_{i}}\left[\mathbb{P}\left(g_{1}>d_{1}^{\alpha_{1}} \Upsilon \Lambda\right)\right]=\mathbb{E}_{g_{i}, d_{i}} \\
& \times\left[\frac{\Gamma\left(m_{1}, \frac{m_{1}}{\mu_{1}} d_{1}^{\alpha_{1}} \Upsilon \Lambda\right)}{\Gamma\left(m_{1}\right)}\right] \\
& =\mathbb{E}_{\Lambda}\left[e^{-\frac{m_{1}}{\mu_{1}} d_{1}^{\alpha_{1}} \Upsilon \Lambda} \sum_{k=0}^{m_{1}-1} \frac{1}{k !} \frac{m_{1}^{k}}{\mu_{1}^{k}}\left(d_{1}^{\alpha_{1}} \Upsilon \Lambda\right)^{k}\right] \\
& =\int_{0}^{+\infty}\left[e^{-\frac{m_{1}}{\mu_{1}} d_{1}^{\alpha_{1}} \Upsilon \Lambda} \sum_{k=0}^{m_{1}-1} \frac{1}{k !} \frac{m_{1}^{k}}{\mu_{1}^{k}}\left(d_{1}^{\alpha_{1}} \Upsilon \Lambda\right)^{k}\right] f_{\Lambda}(\Lambda) \mathrm{d} \Lambda
\end{aligned}
$$

\footnotetext{
${ }^{1}$ When $m$ is an integer, we have the upper incomplete gama function $\Gamma(m, x)=(m-1) ! e^{-x} \sum_{k=0}^{m-1} \frac{x^{k}}{k !}$, the gamma function $\Gamma(m)=(m-1) !$, and $\frac{\Gamma(m, m x)}{\Gamma(m)}=e^{-m x} \sum_{k=0}^{m-1} \frac{m^{k}}{k !} x^{k}[46]$.
} 


$$
\begin{aligned}
& =\sum_{k=0}^{m_{1}-1} \frac{1}{k !}\left(\frac{m_{1} d_{1}^{\alpha_{1}} \Upsilon}{\mu_{1}}\right)^{k} \int_{0}^{+\infty}\left[e^{-\frac{m_{1} d_{1}^{\alpha}{ }^{1} \Upsilon}{\mu_{1}} \Lambda} \Lambda^{k}\right] f_{\Lambda}(\Lambda) \mathrm{d} \Lambda \\
& =\sum_{k=0}^{m_{1}-1} \frac{s^{k}}{k !}(-1)^{k} \frac{\mathrm{d}^{k} \mathcal{L}_{\Lambda}(s)}{\mathrm{d} s^{k}}
\end{aligned}
$$

733 where $f_{\Lambda}(\Lambda)$ represents the p.d.f. of $\Lambda$, and $s \triangleq \frac{m_{1} d_{1}^{\alpha}{ }^{1} \Upsilon}{\mu_{1}}$, and

$\mathcal{L}_{\Lambda}($.$) represents the Laplace transform of the interference plus$ noise of vehicle $v_{0}$, while the last step exploits the property of $x^{n} f(x) \stackrel{\mathcal{L}}{\Longleftrightarrow} \frac{\mathrm{d}^{k} \mathcal{L}_{\Lambda}(s)}{\mathrm{d}_{s}{ }^{k}}$.

The Laplace transform of $\Lambda$ can be calculated as follows:

$$
\begin{aligned}
\mathcal{L}_{\Lambda}(s) & =\mathbb{E}_{\Lambda}\left[e^{-s \Lambda}\right] \\
& =e^{-s \sigma^{2}} \mathbb{E}_{g_{i}, d_{i}}\left[\prod_{v_{i} \in \mathcal{S} \backslash\left\{v_{0}, v_{1}\right\}} e^{-s g_{i} d_{i}^{-\alpha_{2}}}\right] .
\end{aligned}
$$

Since all the vehicles $v_{i}\left(\forall v_{i} \in \mathcal{S} \backslash\left\{v_{0}, v_{1}\right\}\right)$ are independent of each other, all the channel gains $\left\{g_{i}\right\}$ are i.i.d. and their locations generated independently based on the PPP are also i.i.d.; hence, (35) can be rewritten as

$$
\begin{aligned}
\mathcal{L}_{\Lambda}(s) & =e^{-s \sigma^{2}} \mathbb{E}_{d_{i}}\left[\prod_{v_{i} \in \mathcal{S} \backslash\left\{v_{0}, v_{1}\right\}} \mathbb{E}_{g_{i}}\left[e^{-s g_{i} d_{i}^{-\alpha_{2}}}\right]\right] \\
& =e^{-s \sigma^{2}} \mathbb{E}_{d_{i}}\left[\prod_{v_{i} \in \mathcal{S} \backslash\left\{v_{0}, v_{1}\right\}} \frac{1}{1+s \mu_{2} d_{i}^{-\alpha_{2}}}\right] \\
& =e^{-s \sigma^{2}} \exp \left(-\lambda \int_{d_{1}}^{+\infty}\left(1-\frac{1}{1+s \mu_{2} \zeta^{-\alpha_{2}}}\right) 2 W \mathrm{~d} \zeta\right) \\
& =\exp \left(-s \sigma^{2}-2 \lambda W \int_{d_{1}}^{+\infty} \frac{1}{1+\frac{\zeta^{\alpha_{2}}}{\mu_{2} s}} \mathrm{~d} \zeta\right)
\end{aligned}
$$

where the second step is based on the assumption of experiencing a Rayleigh-fading channel with a mean of $\mu_{2}$ between vehicle $v_{i}$ (except for the closest vehicle $v_{1}$ ) and $v_{0}$. To elaborate a little further, the third step follows from the probability generating functional of the PPP [24] and the lower boundary of the integration is $d_{1}$, since the closest vehicle $v_{i}$ imposing interference on vehicle $v_{0}$ should be farther than $v_{0}$ 's peer vehicle $v_{1}$. By invoking the following change of variables $u=\frac{\zeta}{\left(\mu_{2} s\right)^{1 / \alpha_{2}}}$ in (36), we have

$$
\begin{aligned}
\mathcal{L}_{\Lambda}(s) & =\exp \left(-s \sigma^{2}-2 \lambda W\left(\mu_{2} s\right)^{1 / \alpha_{2}} \int_{\frac{d_{1}}{\left(\mu_{2}\right)^{1 / \alpha_{2}}}}^{+\infty} \frac{1}{1+u^{\alpha_{2}}} \mathrm{~d} u\right) \\
& =\exp \left[-s \sigma^{2}-2 \lambda W d_{1} \Phi_{\alpha_{2}}\left(\mu_{2} s d_{1}^{-\alpha_{2}}\right)\right]
\end{aligned}
$$

where $\Phi_{\alpha}(x)$ is as in (12). To summarize, by combining (32), (34), and (37), we arrive at vehicle $v_{0}$ 's channel-induced OP as

$$
\begin{aligned}
p_{0}= & 1-\left.2 \lambda W \int_{d_{1}=0}^{+\infty} \sum_{k=0}^{m_{1}-1} \frac{\left(-m_{1} d_{1}^{\alpha_{2}} \Upsilon\right)^{k}}{k ! \mu_{1}^{k}} \frac{\mathrm{d}^{k} \mathcal{L}_{\Lambda}(s)}{\mathrm{d} s^{k}}\right|_{s=\frac{m_{1} d_{1}^{\alpha_{1}} \Upsilon}{\mu_{1}}} \\
& e^{-2 \lambda W d_{1}} \mathrm{~d} d_{1}
\end{aligned}
$$

with $\mathcal{L}_{\Lambda}(s)$ in (37). By setting $d_{1}=\tau$, we have (11), which 753 completes the proof of Theorem 1.

\section{APPENDIX B}

PROOF OF COROLLARY 1

Since Rayleigh fading is a special case of Nakagami- $m 756$ fading associated with $m=1$, we can calculate vehicle $v_{0}$ 's 757 channel-induced OP in the highway scenario considered by 758 setting $m_{1}=1$ and $\mu_{1}=\mu_{2}=\mu$ in (11), which yields

$$
\begin{aligned}
p_{0} \mathrm{hwy}_{1}= & 1-2 \lambda W \int_{\tau=0}^{+\infty} \mathcal{L}_{\Lambda}\left(\frac{\tau^{\alpha_{1}} \Upsilon}{\mu}\right) e^{-2 \lambda W \tau} \mathrm{d} \tau \\
= & 1-2 \lambda W \int_{\tau=0}^{+\infty} \exp \\
& \times\left(-\frac{\tau^{\alpha_{1}} \Upsilon}{\mu} \sigma^{2}-2 \lambda W \int_{\tau}^{+\infty} \frac{1}{1+\frac{\zeta^{\alpha} 2}{\tau^{\alpha} 1}} \mathrm{~d} \zeta\right) \\
= & e^{-2 \lambda W \tau} \mathrm{d} \tau .
\end{aligned}
$$

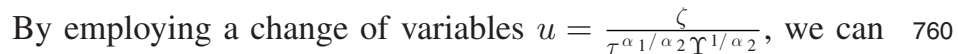
rewrite (39) as

$$
\begin{aligned}
p_{0} \mathrm{hwy}_{1}= & 1-2 \lambda W \int_{\tau=0}^{+\infty} e^{-2 \lambda W \tau-\frac{\tau^{\alpha} 1 \Upsilon}{\mu} \sigma^{2}-2 \lambda W \Upsilon^{\frac{1}{\alpha_{2}}} \tau^{\frac{\alpha_{1}}{\alpha_{2}}}} \\
& \times e^{\mathcal{G}_{\alpha_{2}}\left[\left(\frac{\tau^{\alpha_{2}-\alpha_{1}}}{\Upsilon}\right)^{\frac{1}{\alpha_{2}}}\right]} \mathrm{d} \tau \\
= & 1-2 \lambda W \int_{\tau=0}^{+\infty} \exp \left(-\frac{\sigma^{2} \Upsilon}{\mu} \tau^{\alpha_{1}}\right) \\
& \times \exp \left[-2 \lambda W \Upsilon^{\frac{1}{\alpha_{2}}} \tau^{\frac{\alpha_{1}}{\alpha_{2}}} \mathcal{G}_{\alpha_{2}}\left(\left(\frac{\tau^{\alpha_{2}-\alpha_{1}}}{\Upsilon}\right)^{\frac{1}{\alpha_{2}}}\right)\right] \\
& \cdot e^{-2 \lambda W \tau} \mathrm{d} \tau
\end{aligned}
$$

where according to [47], we have

$$
\begin{aligned}
\mathcal{G}_{\alpha}(x) & =\int_{x}^{+\infty} \frac{1}{1+u^{\alpha}} \mathrm{d} u \\
& =\frac{1}{\alpha-1} \frac{x}{1+x^{\alpha}} \mathbf{F}\left(1,1 ; 2-\frac{1}{\alpha} ; \frac{1}{1+x^{\alpha}}\right)
\end{aligned}
$$

with the hypergeometric function given by $\mathbf{F}(a, b ; c ; z)=763$ $1+\sum_{n=1}^{+\infty} \frac{z^{n}}{n !} \prod_{m=0}^{n-1} \frac{(a+m)(b+m)}{c+m}$. Although (40) appears to 764 be complicated, its physical interpretation is quite clear. The 765 first term $\exp \left(-\frac{\sigma^{2} \Upsilon}{\mu} \tau^{\alpha_{1}}\right)$ within the integration represents the 766 channel-induced OP as a function of noise, the second term 767 $\exp \left[-2 \lambda W \Upsilon^{\frac{1}{\alpha_{2}}} \tau^{\frac{\alpha_{1}}{\alpha_{2}}} \mathcal{G}_{\alpha_{2}}\left(\left(\frac{\tau^{\alpha_{2}-\alpha_{1}}}{\Upsilon}\right)^{\frac{1}{\alpha_{2}}}\right)\right]$ represents the channel- 768 induced OP influenced by the other vehicles $v_{i}$, and the last 769 term $e^{-2 \lambda W \tau}$ is associated with the p.d.f. of the variable $\tau=d_{1} . \quad 770$ This completes the proof of Corollary 1. 
APPENDIX C

PROOF OF COROLLARY 2

By substituting $\alpha_{1}=\alpha_{2}=\alpha$ in (13), we have

$$
\begin{aligned}
p_{0}^{\mathrm{hwy}_{2}=} & 1-2 \lambda W \int_{\tau=0}^{+\infty} \exp \left(-\frac{\sigma^{2} \Upsilon}{\mu} \tau^{\alpha}\right) \\
& \times \exp \left[-2 \lambda W \Upsilon^{\frac{1}{\alpha}} \mathcal{G}_{\alpha}\left(\Upsilon^{-\frac{1}{\alpha}}\right) \tau\right] \cdot e^{-2 \lambda W \tau} \mathrm{d} \tau \\
= & 1-2 \lambda W \int_{\tau=0}^{+\infty} \exp \left(-\frac{\sigma^{2} \Upsilon}{\mu} \tau^{\alpha}\right) \\
& \times \exp \left(-2 \lambda W \Phi_{\alpha}(\Upsilon) \tau\right) \cdot e^{-2 \lambda W \tau} \mathrm{d} \tau \\
= & 1-2 \lambda W \int_{\tau=0}^{+\infty} \exp \\
& \times\left[-\frac{\sigma^{2} \Upsilon}{\mu} \tau^{\alpha}-2 \lambda W\left(1+\Phi_{\alpha}(\Upsilon)\right) \tau\right] \mathrm{d} \tau
\end{aligned}
$$

where the second step is valid according to (12)

$$
\Phi_{\alpha}(\Upsilon)=\Upsilon^{1 / \alpha} \int_{\Upsilon-1 / \alpha}^{+\infty} \frac{1}{1+u^{\alpha}} \mathrm{d} u=\Upsilon^{1 / \alpha} \mathcal{G}_{\alpha}\left(\Upsilon^{-1 / \alpha}\right)
$$

$$
\begin{aligned}
p_{0}^{\mathrm{hwy}_{2}=} & 1-2 \lambda W \int_{\tau=0}^{+\infty} \exp \left(-\frac{\sigma^{2} \Upsilon}{\mu} \tau^{2}\right) \\
& \times \exp \left(-2 \lambda W \Upsilon^{\frac{1}{2}} \mathcal{G}_{2}\left(\Upsilon^{-\frac{1}{2}}\right) \tau\right) \cdot e^{-2 \lambda W \tau} \mathrm{d} \tau \\
= & 1-2 \lambda W \int_{\tau=0}^{+\infty} \exp \\
& \times\left(-\frac{\sigma^{2} \Upsilon}{\mu} \tau^{2}-2 \lambda W(1+\sqrt{\Upsilon} \arctan \sqrt{\Upsilon}) \tau\right) \mathrm{d} \tau \\
= & 1-2 \lambda W \sqrt{\frac{\pi}{\chi_{1}(\Upsilon)}} \exp \left(\frac{\chi_{2}^{2}(\Upsilon)}{4 \chi_{1}(\Upsilon)}\right) \\
& \times Q\left(\frac{\chi_{2}(\Upsilon)}{\sqrt{2 \chi_{1}(\Upsilon)}}\right)
\end{aligned}
$$

780 where the second step is valid because $\arctan (1 / u)=$ $781 \int_{u}^{+\infty} \frac{1}{1+u^{2}} \mathrm{~d} u$ and the last step exploits the following exponential 782 integration properties [46]:

$$
\int_{\tau=0}^{+\infty} \exp \left(-a \tau^{2}-b \tau\right) \mathrm{d} \tau=\sqrt{\frac{\pi}{a}} \exp \left(\frac{b^{2}}{4 a}\right) Q\left(\frac{b}{\sqrt{2 a}}\right)
$$

783 with the $Q$-function given by $Q(x)=\frac{1}{2 \pi} \int_{x}^{+\infty} \exp \left(-y^{2} / 2\right) \mathrm{d} y$. 784

This completes the proof of Corollary 2 .

\section{REFERENCES}

[1] S. Panichpapiboon and W. Pattara-Atikom, "A review of information dissemination protocols for vehicular ad hoc networks," IEEE Commun. Surveys Tuts., vol. 14, no. 3, pp. 784-798, Mar. 2012.

[2] D. Williams, "The arbitron national in-car study," [Online]. Available: http://www.arbitron.com/downloads/InCarStudy2009.pdf

[3] J. Zhao, Y. Zhang, and G. Cao, "Data pouring and buffering on the road: A new data dissemination paradigm for vehicular ad hoc networks," IEEE Trans. Veh. Technol., vol. 56, no. 6, pp. 3266-3277, Nov. 2007.

[4] J. Santa and A. Gomez-Skarmeta, "Sharing context-aware road and safety information," IEEE Pervasive Comput., vol. 8, no. 3, pp. 58-65, Jul. 2009.

[5] Y. Fallah, C.-L. Huang, R. Sengupta, and H. Krishnan, "Analysis of information dissemination in vehicular ad-hoc networks with application to cooperative vehicle safety systems," IEEE Trans. Veh. Technol., vol. 60, no. 1, pp. 233-247, Jan. 2011.

[6] N. Cenerario, T. Delot, and S. Ilarri, "A content-based dissemination protocol for vanets: Exploiting the encounter probability," IEEE Trans. Intell. Transp. Syst., vol. 12, no. 3, pp. 771-782, Sep. 2011.

[7] F. Ros, P. Ruiz, and I. Stojmenovic, "Acknowledgment-based broadcast protocol for reliable and efficient data dissemination in vehicular ad hoc networks," IEEE Trans. Mobile Comput., vol. 11, no. 1, pp. 33-46, Jan. 2012.

[8] M. Fogue, P. Garrido, F. Martinez, J. Cano, C. Calafate, and P. Manzoni, "An adaptive system based on roadmap profiling to enhance warning message dissemination in vanets," IEEE/ACM Trans. Netw., vol. 21, no. 3, pp. 883-895, Jun. 2013.

[9] N. Kumar and J.-H. Lee, "Peer-to-peer cooperative caching for data dissemination in urban vehicular communications," IEEE Syst. J., vol. 8, no. 4, pp. 1136-1144, Dec. 2014. "Optimizing content dissemination in vehicular networks with radio het- 815 erogeneity," IEEE Trans. Mobile Comput., vol. 13, no. 6, pp. 1312-1325, 816 Jun. 2014.

, S. Aissa, and M. Mehmet-Ali, "Performance modeling of message dissemination in vehicular ad hoc networks with priority," IEEE J. Sel. Areas Commun., vol. 29, no. 1, pp. 61-71, Jan. 2011.

[12] Q. Wang, J. Hu, and J. Zhang, "Performance evaluation of information propagation in vehicular ad hoc network," IET Intell. Transport Syst., vol. 6, no. 2, pp. 187-196, Jun. 2012.

[13] K. Rostamzadeh and S. Gopalakrishnan, "Analysis of message dissemination in vehicular networks," IEEE Trans. Veh. Technol., vol. 62, no. 8, pp. 3974-3982, Oct. 2013.

[14] J. Hu, L.-L. Yang, and L. Hanzo, "Cooperative multicast aided picocellular hybrid information dissemination in mobile social networks: Delay/energy evaluation and relay selection," in Proc. IEEE Wirel. Commun. Netw. Conf., Istanbul, Turkey, Apr. 2014, pp. 3207-3212.

[15] J. Hu, L.-L. Yang, and L. Hanzo, "Throughput and delay analysis of wireless multicast in distributed mobile social networks based on geographic social relationships," in Proc. IEEE Wirel. Commun. Netw. Conf., Istanbul, Turkey, Apr. 2014, pp. 1874-1879.

16] S. Dietzel, J. Petit, G. Heijenk, and F. Kargl, "Graph-based metrics for 835 insider attack detection in vanet multihop data dissemination protocols," 836 IEEE Trans. Veh. Technol., vol. 62, no. 4, pp. 1505-1518, May 2013.

[17] K. Rostamzadeh, H. Nicanfar, N. Torabi, S. Gopalakrishnan, and V. Leung, 838 "A context-aware trust-based information dissemination framework for 839 vehicular networks," IEEE Internet Things J., vol. 2, no. 2, pp. 121-132, 840 Apr. 2015.

[18] M. Fogue et al., "Securing warning message dissemination in vanets using cooperative neighbor position verification," IEEE Trans. Veh. Technol., vol. 64 , no. 6 , pp. $2538-2550$, Jun. 2015.

[19] T. Luan, R. Lu, X. Shen, and F. Bai, "Social on the road: Enabling secure and efficient social networking on highways," IEEE Wirel. Commun., vol. 22, no. 1, pp. 44-51, Feb. 2015.

[20] T. Luan, X. Shen, F. Bai, and L. Sun, "Feel bored? Join verse! Engineering 848 vehicular proximity social networks," IEEE Trans. Veh. Technol., vol. 64, 849 no. 3, pp. 1120-1131, Mar. 2015.

[21] J. Hu, L.-L. Yang, and L. Hanzo, "Distributed multistage cooperative- 851 social-multicast-aided content dissemination in random mobile networks," 852 IEEE Trans. Veh. Technol., vol. 64, no. 7, pp. 3075-3089, Jul. 2015.

[22] J. G. Andrews, F. Baccelli, and R. K. Ganti, "A tractable approach to 854 coverage and rate in cellular networks," IEEE Trans. Commun., vol. 59, 855 no. 11, pp. 3122-3134, Nov. 2011.

[23] H. Zhang, S. Chen, L. Feng, Y. Xie, and L. Hanzo, "A universal approach 857 to coverage probability and throughput analysis for cellular networks," 858 IEEE Trans. Veh. Technol., vol. 64, no. 9, pp. 4245-4256, Sep. 2015.

\section{6} 88
796 98 99 800 01 803
804
805 806 806 08 809 11 12 14 17 818
819 20 21 83
824 25 86
827 828 829
830
831 . . 34 (a) (a) 41 ,

.
55 857
858 859 
[24] R. K. Ganti and M. Haenggi, "Interference and outage in clustered wireless ad hoc networks," IEEE Trans. Inf. Theory, vol. 35, no. 9, pp. 4067-4086, Sep. 2009.

[25] N. Alam, A. T. Balaei, and A. G. Dempster, "A DSRC doppler-based cooperative positioning enhancement for vehicular networks with gps availability," IEEE Trans. Veh. Technol., vol. 60, no. 9, pp. 4462-4470, Nov. 2011.

[26] P. Sujit, D. Lucani, and J. Sousa, "Bridging cooperative sensing and route planning of autonomous vehicles," IEEE Trans. Veh. Technol., vol. 30, no. 5, pp. 912-922, Jun. 2012.

[27] K. Xu, M. Gerla, and S. Bae, "How effective is the IEEE 802.11 RTS/CTS handshake in ad hoc networks," in Proc. IEEE Global Telecommun. Conf., Taipei, Taiwan, Nov. 2002, vol. 1, pp. 72-76.

[28] C. Jiang, H. Zhang, Y. Ren, and H. H. Chen, "Energy-efficient noncooperative cognitive radio networks: micro, meso, and macro views," IEEE Commun. Mag., vol. 52, no. 7, pp. 14-20, Jul. 2014.

[29] P. Gupta and P. Kumar, "The capacity of wireless networks," IEEE Trans. Inf. Theory, vol. 46, no. 2, pp. 388-404, Mar. 2000.

[30] L. Cheng, B. Henty, D. Stancil, F. Bai, and P. Mudalige, "Mobile vehicleto-vehicle narrow-band channel measurement and characterization of the $5.9 \mathrm{GHz}$ dedicated short range communication (DSRC) frequency band," IEEE J. Sel. Area Commun., vol. 25, no. 8, pp. 1501-1516, Oct. 2007.

[31] J. Hu, L.-L. Yang, and L. Hanzo, "Maximum average service rate and optimal queue scheduling of delay-constrained hybrid cognitive radio in Nakagami fading channels," IEEE Trans. Veh. Technol., vol. 62, no. 5, pp. 2220-2229, Jun. 2013.

[32] R. S. Sutton and A. G. Barto, Reinforcement Learning: An Introduction. Cambridge, MA, USA: MIT Press, 1998.

[33] M. Bennis, S. M. Perlaza, P. Blasco, Z. Han, and H. V. Poor, "Selforganization in small cell networks: A reinforcement learning approach," IEEE Trans. Wirel. Commun., vol. 12, no. 7, pp. 3202-3212, Jul. 2013.

[34] C. Jiang, Y. Chen, and K. J. R. Liu, "Multi-channel sensing and access game: Bayesian social learning with negative network externality," IEEE Trans. Wirel. Commun., vol. 13, no. 4, pp. 2176-2188, Apr. 2014.

[35] F. L. Lewis, D. Vrabie, and K. G. Vamvoudakis, "Reinforcement learning and feedback control: Using natural decision methods to design optimal adaptive controllers," IEEE Control Syst. Mag., vol. 32, no. 6, pp. 76-105, Dec. 2012.

[36] T. Matsui, T. Goto, K. Izumi, and Y. Chen, "Compound reinforcement learning: Theory and an application to finance," in Recent Advances in Reinforcement Learning (Lecture Notes in Computer Science). Berlin, Germany: Springer, 2012, pp. 321-332.

[37] R. M. Jones et al., "Behavioral and neural properties of social reinforcement learning," J. Neurosci., vol. 31, no. 37, pp. 13039-13045, Sep. 2011.

[38] C. Jiang, Y. Chen, Y. Gao, and K. J. R. Liu, "Indian buffet game with negative network externality and non-bayesian social learning," IEEE Trans. Syst., Man, Cybern., Syst., vol. 45, no. 4, pp. 609-623, Apr. 2015.

[39] J. Yuan et al., "T-drive: Driving directions based on taxi trajectories," in Proc. 18th SIGSPATIAL Int. Conf. Adv. Geographic Inf. Syst., Nov. 2010, pp. 99-108.

[40] E. Giordano, E. D. Sena, G. Pau, and M. Gerla, "Vergilius: A scenario generator for vanet," in IEEE 71st Veh. Technol. Conf., Taipei, Taiwan, May 2010, pp. 1-5.

[41] M. Cesana, L. Fratta, M. Gerla, E. Giordano, and G. Pau, "C-vet the ucla campus vehicular testbed: Integration of vanet and mesh networks," in Proc. Eur. Wirel. Conf., Lucca, Italy, Apr. 2014, pp. 689-695.

[42] E. Giordano, R. Frank, G. Pau, and M. Gerla, "Cooperative multicast aided picocellular hybrid information dissemination in mobile social networks: Delay/energy evaluation and relay selection,"' in Proc. 7th Int. Conf. Wirel. On-demand Netw. Syst. Serv., Kranjska Gora, Slovenia, Feb. 2010, pp. 57-60.

[43] C. Sommer, D. Eckhoff, and F. Dressler, "IVC in cities: Signal attenuation by buildings and how parked cars can improve the situation," IEEE Trans. Mobile Comput., vol. 13, no. 8, pp. 1733-1745, Aug. 2014.

[44] C. Sommer, R. German, and F. Dressler, "Bidirectionally coupled network and road traffic simulation for improved IVC analysis," IEEE Trans. Mobile Comput., vol. 10, no. 1, pp. 3-15, Jan. 2011.

[45] C. Sommer and F. Dressler, "Progressing toward realistic mobility models in VANET simulations," IEEE Commun. Mag., vol. 46, no. 11, pp. 132-137, Nov. 2008.

[46] I. S. Gradshteyn and I. M. Ryzhik, Table of Integrals, Series, Products. New York, NY, USA: Academic, 2007

[47] S. Mukherjee, "Distribution of downlink sinr in heterogeneous cellular networks," IEEE J. Sel. Area Commun., vol. 30, no. 3, pp. 575-585, Apr. 2012.

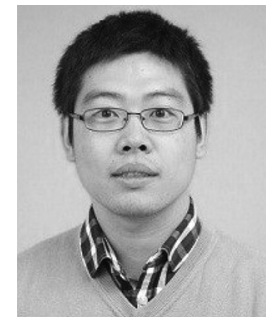

Chunxiao Jiang (S'09-M'13-SM'15) received the B.S. (Hons.) degree in information engineering from Beihang University, Beijing, China, in 2008 and the Ph.D. (Hons.) degree in electronic engineering from Tsinghua University, Beijing, in 2013

He is currently an Assistant Research Fellow with the Tsinghua Space Center, Tsinghua University. His research interests include the application of game theory, optimization, and statistical theories to communication, networking, signal processing, and resource allocation problems, in particular space information networks, heterogeneous networks, social networks, and big data privacy. He has authored/co-authored $100+$ technical papers in renowned international journals and conferences, including 50+ renowned IEEE journal papers.

Dr. Jiang has been actively involved in organizing and chairing sessions and has served as a member of the technical program committee, as well as the Symposium/Workshop Chair for a number of international conferences. He is currently an Editor of the Wiley Wireless Communications and Mobile Comput ing, Wiley Security and Communications Networks, the International Journa of Big Data Intelligence, and a Guest Editor of ACM/Springer Mobile Network \& Applications Special Issue on "Game Theory for 5G Wireless Networks." He received the Best Paper Award from IEEE GLOBECOM in 2013, the Bes Student Paper Award from IEEE GlobalSIP in 2015, the Distinguished Dissertation Award from the Chinese Association for Artificial Intelligence (CAAI) in 2014, and the Tsinghua Outstanding Postdoc Fellow Award (only ten winners each year) in 2015.

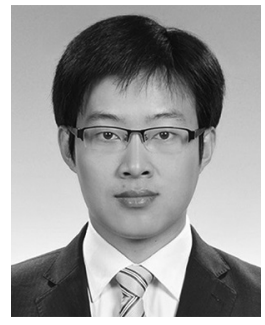

Haijun Zhang (M'13) received the Ph.D. degree from Beijing University of Posts Telecommunications (BUPT), Beijing, China.

$\mathrm{He}$ is a Postdoctoral Research Fellow with the Department of Electrical and Computer Engineering, University of British Columbia, Vancouver, BC, Canada. From September 2011 to September 2012, he visited the Centre for Telecommunications Research, King's College London, London, U.K., as a Visiting Research Associate, supported by the China Scholarship Council. He has published more than 70 papers and has authored two books. He serves as an Editor of Journal of Network and Computer Applications, Wireless Networks, Telecommunication Systems, and the KSII Transactions on Internet and Information Systems. He also has served as the Leading Guest Editor of ACM/Springer Mobile Networks \& Applications (MONET) Special Issue on "Game Theory for 5G Wireless Networks." He has served as the General Chair of GameNets'16, 5GWN2017, and the ICC2017 Workshop on 5GUDN and served as the Symposium Chair of the GameNets'14 and Track Chair of ScalCom'15. His current research interests include 5G resource allocation, NOMA, LTE-U, heterogeneous small cell networks, and ultra-dense networks.

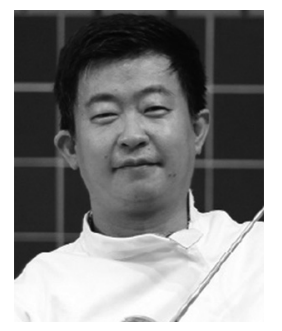

Zhu Han (S'01-M'04-SM'09-F'14) received the B.S. degree in electronic engineering from Tsinghua University, Beijing, China, in 1997 and the M.S. and Ph.D. degrees in electrical and computer engineering from the University of Maryland, College Park, MD, USA, in 1999 and 2003, respectively.

From 2000 to 2002, he was an R\&D Engineer with JDSU, Germantown, MD. From 2003 to 2006, he was a Research Associate with the University of Maryland. From 2006 to 2008, he was an Assistant Professor with Boise State University, Boise, ID, USA $\mathrm{He}$ is currently a Professor with Electrical and Computer Engineering Department, as well as with the Computer Science Department, University of Houston Houston, TX, USA. His research interests include wireless resource allocation and management, wireless communications and networking, game theory, big data analysis, security, and smart grid.

Dr. Han received the National Science Foundation Career Award in 2010, 1001 the Fred W. Ellersick Prize from the IEEE Communication Society in 2011, the 1002 EURASIP Best Paper Award for the Journal on Advances in Signal Processing 1003 in 2015, the IEEE Leonard G. Abraham Prize in the field of Communications 1004 Systems (best paper award in IEEE JSAC) in 2016, and several best paper 1005 awards at IEEE conferences. He is currently an IEEE Communications Society 1006 Distinguished Lecturer.

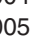




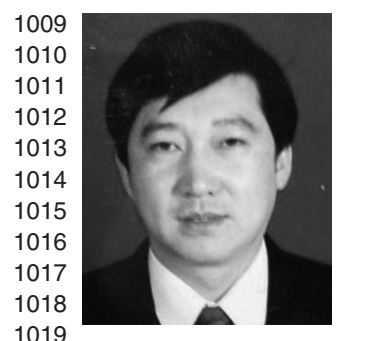

Yong Ren (SM'16) received the B.S., M.S., and Ph.D. degrees in electronic engineering from Harbin Institute of Technology, Harbin, China, in 1984, 1987, and 1994, respectively.

He was a Postdoctoral Researcher with the Department of Electronics Engineering, Tsinghua University, Beijing, China, from 1995 to 1997 . He is currently a Professor with the Department of Electronics Engineering and the Director of the Complexity Engineered Systems Lab, Tsinghua University. He holds 12 patents and has authored or co-authored 1020 more than 100 technical papers in the behavior of computer networks, peer-to1021 peer networks, and cognitive networks. His current research interests include 1022 complex systems theory and its applications to the optimization and information 1023 sharing of the Internet, Internet of things and ubiquitous networks, cognitive 1024 networks, and cyber-physical systems.

1025

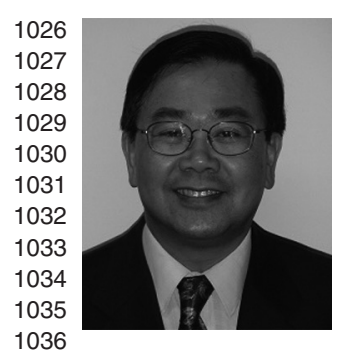

Victor C. M. Leung (S'75-M'89-SM'97-F'03) received the B.A.Sc. (Hons.) and Ph.D. degrees in electrical engineering from the University of British Columbia (UBC), Vancouver, BC, Canada, in 1977 and 1982, respectively. He received the Natural Sciences and Engineering Research Council Postgraduate Scholarship for his Ph.D. research.

From 1981 to 1987, he was a Senior Member of Technical Staff and satellite system specialist at MPR Teltech Ltd., Canada. In 1988, he was a Lecturer with the Department of Electronics, Chinese University of 1037 Hong Kong, Sha Tin, Hong Kong. He joined the UBC as a Faculty Member in 10381989 and is currently a Professor and the TELUS Mobility Research Chair of 1039 Advanced Telecommunications Engineering with the Department of Electrical 1040 and Computer Engineering. He has co-authored more than 900 technical papers 1041 in international journals and conference proceedings, 31 book chapters, and has 1042 co-edited 11 book titles. Several of his papers have been selected for best paper 1043 awards. His research interests include the areas wireless networks and mobile 1044 systems.

1045 Dr. Leung is a registered Professional Engineer in the Province of British 1046 Columbia, Canada. He is a Fellow of the Royal Society of Canada, the En1047 gineering Institute of Canada, and the Canadian Academy of Engineering. He 1048 was a Distinguished Lecturer of the IEEE Communications Society. He is a 1049 member of the editorial boards of IEEE WIRELESS COMMUNICATIONS LETTERS, 1050 the IEEE Journal ON SELECTEd AREAS IN COMMUNICATIONS Series on Green 1051 Communications and Networking, the IEEE TRANSACTIONS ON GREEN COM1052 MUNICATIONS AND NETWORKING, IEEE ACCESS, Computer Communications, 1053 and several other journals and has previously served on the editorial boards of 1054 the IEEE JOURNAL ON SELECTED AREAS IN COMMUNICATIONS-Wireless Com1055 munications Series, the IEEE TRANSACTIONS ON WIRELESS COMMUNICATIONS, 1056 the IEEE TRANSACTIONS ON VEHICULAR TEChNOLOGY, the IEEE TRANSAC1057 TIONS ON COMPUTERS, and the Journal of Communications and Networks. He 1058 has guest-edited many journal special issues and has provided leadership to the 1059 organizing committees and technical program committees of numerous con1060 ferences and workshops. He received the IEEE Vancouver Section Centennial 1061 Award and the 2012 UBC Killam Research Prize, as well as the APEBC Gold 1062 Medal as the head of the graduating class of the Faculty of Applied Science at 1063 UBC.

1064

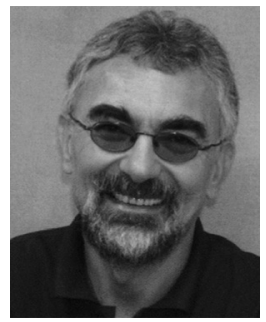

Lajos Hanzo (F'08) received the M.S. degree in elec- 1065 tronics and the Ph.D. degree from the Technical Uni- 1066 versity of Budapest, Budapest, Hungary, in 1976 and 1067 1983, respectively. He received the prestigious Doc- 1068 tor of Sciences research degree in wireless commu- 1069 nications from the University of Southampton, U.K., 1070 in 2004.

In 2016, he was admitted to the Hungarian 1072 Academy of Science, Budapest, Hungary. During his 1073 40-year career in telecommunications, he has held 1074 various research and academic posts in Hungary, 1075 Germany, and the U.K. Since 1986, he has been with the School of Electron- 1076 ics and Computer Science, University of Southampton, U.K., where he holds 1077 the Chair in telecommunications. He has successfully supervised $111 \mathrm{Ph} . \mathrm{D} .1078$ students, coauthored 20 John Wiley/IEEE Press books on mobile radio com- 1079 munications, totalling in excess of 10000 pages, published $1600+$ research 1080 contributions on IEEE Xplore, acted both as Technical Program Committee 1081 member and General Chair of IEEE conferences, presented keynote lectures, 1082 and received a number of distinctions. Currently he is directing a 60-strong 1083 academic research team, working on a range of research projects in the field 1084 of wireless multimedia communications sponsored by industry; the Engineer- 1085 ing and Physical Sciences Research Council (EPSRC), U.K.; and the European 1086 Research Council's Advanced Fellow Grant. He is an enthusiastic supporter of 1087 industrial and academic liaison, and he offers a range of industrial courses. He 1088 has $25000+$ citations and an H-index of 60. For further information on research 1089 in progress and associated publications, see http://www-mobile.ecs.soton.ac.uk. 1090 Dr. Hanzo is also a Governor of the IEEE Vehicular Technology Society. Dur- 1091 ing 2008-2012, he was the Editor-in-Chief of the IEEE Press and a Chaired 1092 Professor with Tsinghua University, Beijing, China. In 2009, he received an 1093 honorary doctorate award by the Technical University of Budapest and in 2015, 1094 from the University of Edinburgh, Edinburgh, U.K., as well as the Royal Soci- 1095 ety's Wolfson Research Merit Award. He is a Fellow of the Royal Academy of 1096 Engineering, The Institution of Engineering and Technology, and EURASIP. 1097 
Q1. Author: Please provide missing year for Ref. [2]. 